FEDERAL RESERVE BANK OF SAN FRANCISCO

WORKING PAPER SERIES

\title{
The Social Cost of Near-Rational Investment
}

\author{
Tarek A. Hassan \\ University of Chicago \\ Thomas M. Mertens \\ Federal Reserve Bank of San Francisco \\ August 2016 \\ Working Paper 2016-16 \\ http://www.frbsf.org/economic-research/publications/working-papers/wp2016-16.pdf
}

\section{Suggested citation:}

Hassan, Tarek A., Thomas M. Mertens. 2016. “The Social Cost of Near-Rational Investment.” Federal Reserve Bank of San Francisco Working Paper 2016-16.

http://www.frbsf.org/economic-research/publications/working-papers/wp2016-16.pdf

The views in this paper are solely the responsibility of the authors and should not be interpreted as reflecting the views of the Federal Reserve Bank of San Francisco or the Board of Governors of the Federal Reserve System. 


\title{
The Social Cost of Near-Rational Investment*
}

\author{
Tarek A. Hassan ${ }^{\dagger} \quad$ Thomas M. Mertens ${ }^{\ddagger}$
}

July 2016

\begin{abstract}
We show that the stock market may fail to aggregate information even if it appears to be efficient, and that the resulting decrease in the information content of prices may drastically reduce welfare. We solve a macroeconomic model in which information about fundamentals is dispersed and households make small, correlated errors when forming expectations about future productivity. As information aggregates in the market, these errors amplify and crowd out the information content of stock prices. When prices reflect less information, the conditional variance of stock returns rises, causing an increase in uncertainty and costly distortions in consumption, capital accumulation, and labor supply.
\end{abstract}

JEL classification: E2, E3, D83, G1

${ }^{*}$ We thank Daron Acemoglu, Hengjie Ai, George Akerlof, Manuel Amador, Gadi Barelvy, Jeff Campbell, John Y. Campbell, V.V. Chari, John Cochrane, George Constantinides, Martin Eichenbaum, Emmanuel Farhi, Nicola Fuchs-Schündeln, Martin Hellwig, Anil Kashyap, Ralph Koijen, Kenneth L. Judd, David Laibson, John Leahy, Guido Lorenzoni, N. Gregory Mankiw, Lasse Pedersen, Kenneth Rogoff, Andrei Shleifer, Jeremy Stein, Pietro Veronesi, and Pierre-Olivier Weill for helpful comments. We also thank seminar participants at Harvard University, Stanford, Princeton, the University of Chicago, UC Berkeley, the University of British Columbia, the Federal Reserve Bank of Minneapolis, the London School of Economics, the Max Planck Institute Bonn, Goethe University Frankfurt, the University of Mannheim, Oxford, the University of Kansas, the EEA/ESEM, the SED annual meeting, the AEA annual meeting, Econometric Society NASM, the WFA annual meeting, and the NBER $\mathrm{AP}$ and EFCE meetings for valuable discussions. All mistakes remain our own.

${ }^{\dagger}$ University of Chicago, Booth School of Business; Postal Address: 5807 S Woodlawn Avenue, Chicago IL 60637, USA; E-mail: tarek.hassan@chicagobooth.edu.

${ }^{\ddagger}$ Federal Reserve Bank of San Francisco; Postal Address: 101 Market Street, Mail stop 1130, San Francisco, CA 94105, USA; E-mail: thomas.mertens@sf.frb.org. 
Efficient markets incorporate all available information into asset prices. As a result, investors can learn from equilibrium prices and update their expectations accordingly. But if investors learn from equilibrium prices, anything that moves prices has an impact on expectations held by all market participants. We explore the implications of this basic dynamic in a world in which people are less than perfect - a world in which they have a small propensity to make correlated errors when forming their expectations about the future.

We find that relaxing the rational paradigm in this minimal way has important consequences for financial markets, capital accumulation, and welfare: if information is dispersed across investors, the private return to making diligent investment decisions is orders of magnitude lower than the social return. If we allow for individuals to make small common errors in their investment decisions, information aggregation endogenously breaks down precisely when it is most socially valuable (i.e., when private information is highly dispersed). This endogenous informational inefficiency results in increased uncertainty and socially costly distortions in capital accumulation, labor supply, output, and consumption.

We begin by deriving the main economic insights of our analysis in a static model in which households can store their wealth for future consumption or invest it in capital (stocks). The number of consumption goods produced by each unit of capital depends on the realization of a future productivity shock. When making its investment decision, each household observes the equilibrium stock price and a private signal about this realization.

If all households are perfectly rational, the stock price is an effective aggregator of information - it becomes perfectly revealing about all the information contained in the private signals (Grossman, 1976). As a result, the conditional variance of stock returns is low (zero if the noise in private signals is purely idiosyncratic), and households install the optimal amount of capital in equilibrium.

We then show that the economy behaves very differently if households are near-rational rather than fully rational. Near-rational households have an economically small propensity to make small, cross-sectionally correlated, errors when forming their expectations about future productivity. That is, they are slightly too optimistic in some states of the world and slightly too pessimistic in others, but, on average, have unbiased expectations. Households have the option to avoid loading on these near-rational errors by paying a small mental cost (thinking harder), but rationally choose not to do so in equilibrium, because making small errors in the formation of their expectations is almost costless from the perspective of an individual household. (This result follows directly from the envelope theorem.)

A large literature in behavioral finance has developed a wide range of psychologically founded mechanisms that prompt households to make common errors in their investment decisions. ${ }^{1}$ We

\footnotetext{
${ }^{1}$ Some examples are Odean (1998), Odean (1999), Daniel, Hirshleifer, and Subrahmanyam (2001), Barberis,
} 
thus remain open to many possible interpretations of the small common errors households make in our model. The idea is simply that households make small mistakes, and the integral over these mistakes has a non-degenerate distribution.

Although households have little incentive to avoid near-rational errors in their behavior, these errors entail a first-order cost to society because they have an external effect on the market's capacity to aggregate information. When the average household is slightly too optimistic, it wants to invest slightly more of its wealth in stocks, and the stock price must rise. Households that observe this higher stock price may interpret it as either a result of errors made by their peers or, with some probability, a reflection of more positive information about future productivity received by other market participants. Rationally, households thus revise their expectations of future productivity upwards whenever they see a rise in the stock price. As households revise their expectations upward, the stock price must rise further, triggering yet another revision in expectations, and so on. Small errors in the expectation of the average household may thus lead to much larger, non-fundamental, deviations in the equilibrium stock price.

Because the stock price now transmits amplified noise, the elasticity of the stock price with respect to information about the fundamental falls, reducing the information content of stock prices and inhibiting the ability of all households, regardless of whether they are rational or near-rational, to learn about the future. This rise in uncertainty affects social welfare through three distinct channels. First, it distorts the allocation of aggregate quantities, for example, by reducing the covariance of investment with productivity and by distorting the level of capital accumulation. Second, it raises the level of consumption risk individual households face, by generating a dispersion in equilibrium consumption: because the stock price no longer transmits all available information, households differ in their equilibrium actions, adding a cross-sectional component to consumption risk. Third, if households have a preference for early resolution of uncertainty, it also directly lowers welfare by delaying the resolution of uncertainty.

All of these effects of near-rational behavior result from a simple information externality: an individual household does not internalize that making a small error that is correlated with the common error affects other households' ability to learn about the future. We argue this externality provides a logically coherent rationale for why equilibrium asset prices and other economic aggregates might contain non-fundamental noise even if all economic actors appear to act rationally and financial markets are efficient in the sense that rational investors cannot systematically outperform the market.

The more dispersed private information is across households, the more severe are the effects of this information externality, and the more social and private incentives diverge. Because our model features only a single class of almost perfectly rational households, we are able to conduct

Shleifer, and Vishny (1998), Bikhchandani, Hirshleifer, and Welch (1998), Hong and Stein (1999), Allen and Gale (2003), and Hassan and Mertens (2011). 
normative analysis and measure social incentives simply by calculating a utilitarian social welfare function. Using this function, we can show the market's capacity to aggregate information is most socially valuable when private information is most dispersed (when the private signal contains a lot of idiosyncratic noise). However, the lower the informativeness of the private signal, the larger the weight households put on the stock price when forming their expectations, and the larger the amplification of any given near-rational error. The market's capacity to aggregate information is thus most vulnerable to near-rational behavior precisely when it is most socially valuable. By contrast, the private cost of near-rational behavior is decreasing in the dispersion of private information, because a larger amplification of near-rational errors makes distinguishing noise from information more difficult, and thus decreases the return to behaving fully rationally. Making near-rational errors is thus cheapest precisely when they are most socially harmful.

The second part of the paper quantifies the amount of non-fundamental noise and the welfare effects near-rational behavior may entail, by introducing dispersed private information into a state-of-the-art real business cycle (RBC) model with recursive utility, endogenous capital accumulation, and flexible labor supply (Croce, 2014). At the beginning of each period, households receive private signals about the future realization of a temporary and a persistent shock to total factor productivity (TFP). As in the static model, households can condition their expectations on the state of the economy (prices and macroeconomic aggregates) and make small correlated errors when forming their expectations about the realizations of the two productivity shocks. To distinguish the effects of amplified near-rational errors from the effects of noise induced through the signal structure, we also allow for aggregate noise in private signals and the existence of exogenous noisy public signals about the two shocks.

We estimate the model to match standard macroeconomic and asset-pricing moments, as well as an additional set of moments (generated from the Survey of Professional Forecasters) that characterize the dispersion and dynamics of expectations. The model performs well in matching these moments and, in particular, two salient stylized facts highlighted in the recent literature: that disagreement is counter-cyclical (Bloom, 2014; Patton and Timmermann, 2010) and highly persistent $(\mathrm{Yu}, 2011)$.

The first main finding from our estimation is that most of the information about future productivity that households receive originates from noisy private rather than public signals. The reason is that, in the data, differences in opinion about future GDP growth are large relative to the size of forecast errors, suggesting households differ in the signals they receive. Prices (or other economic aggregates households may observe) thus play a potentially important role in aggregating this information.

However our estimates also suggest that, in equilibrium, about half of the information contained in private signals is driven out of asset prices by amplified near-rational errors. Neverthe- 
less, even these noisy asset prices transmit enough information to reduce the posterior variance of the persistent and temporary shocks to TFP by $51 \%$ and $44 \%$, respectively. By contrast, we find that the exogenous public signals are relatively less informative (reducing the posterior variance by $6 \%$ and $20 \%$, respectively). Thus, most of what households learn about the future they learn from endogenously noisy asset prices.

As they distort equilibrium asset prices, amplified near-rational errors also affect all other household decisions, distorting the dynamics of capital accumulation, consumption, labor supply, and output by preventing forward-looking adjustments, introducing non-fundamental variation, and causing a dispersion in consumption and labor supply across households.

We quantify the social cost of near-rational behavior as the percentage rise in consumption that would make households indifferent between remaining in an economy in which all other households behave near-rationally and transitioning to the stochastic steady state of an economy in which all other households behave fully rationally until the end of time. In our benchmark estimation, these social costs amount to $5.31 \%$ of permanent consumption, whereas the incentive to an individual household to avoid small common errors in its expectations is economically small ( $0.13 \%$ of lifetime consumption). The majority of this social cost arises because the delayed resolution of uncertainty directly lowers households' utility, while the increase in consumption risk due to dispersion in equilibrium consumption and leisure across households accounts for an additional 1.5 percentage points of the total social cost.

We make three main caveats to our results. First, our paper offers a near-rational microfoundation for noisy demand. We believe this microfoundation is useful because it is broadly applicable, facilitates welfare analysis, and makes contact with a growing behavioral finance literature. However, other modeling strategies (involving for example liquidity shocks) may yield similar results. Second, although we refer to the information content of asset prices, we do so mainly in keeping with a convention in the literature, where asset prices are generally regarded as being more easily observable than other aggregate variables. In our quantitative model, however, macroeconomic quantities may transmit the same information as asset prices. In this sense, asset prices are not special and we have no way to distinguish learning from asset prices from learning from other aggregate variables. Third, we abstract from the possibility that households can actively acquire information. Grossman and Stiglitz (1980) point out that noise in asset prices may not only reduce information revelation but also improve informational efficiency by inducing households to acquire more private information. All our results are contingent on an exogenous signal structure and do not consider this other, potentially stabilizing, force.

Related Literature Our paper relates to a large literature on noisy rational expectations following Hellwig (1980) and Diamond and Verrecchia (1981) in which exogenous noise trading impedes the aggregation of dispersed information. ${ }^{2}$ In particular, the information exter-

\footnotetext{
${ }^{2}$ Most closely related are Wang (1994), where noise in asset prices arises endogenously from time-varying private
} 
nality we identify is reminiscent of the free-rider problem in Grossman and Stiglitz (1980) where households do not internalize all the benefits of the private information they acquire. Relative to this literature, we make progress on two dimensions. First, the introduction of near-rational behavior microfounds the amount of noise in asset prices in a way that is consistent with the rational paradigm and lends itself to normative analysis. Second, we show that a given amount of near-rational errors has a more detrimental effect on the aggregation of information when information is more dispersed. ${ }^{3}$

A more recent literature on pathologies in information aggregation in financial markets has focused on information externalities arising either from strategic complementarities or from higher-order uncertainty. ${ }^{4}$ Examples of the former include Amador and Weill (2012) and Goldstein, Ozdenoren, and Yuan (2013) who study models in which individuals overweight public signals, amplifying noise contained therein. Examples of the latter include Allen, Morris, and Shin (2006), Bacchetta and Van Wincoop (2008), and Qiu and Wang (2010), where higher-order expectations drive a wedge between asset prices and their fundamentals. Our paper highlights a third type of information externality that arises even when no strategic complementarities are present and first-order expectations fully determine asset prices: ${ }^{5}$ individuals do not internalize how errors in their investment decisions affect others' equilibrium expectations. Pathologies similar to those outlined in this paper are thus likely to arise in any setting in which households observe prices that aggregate dispersed information. Mertens (2009) derives policies that may improve welfare in these settings.

We also contribute to a large literature that studies the general equilibrium effects and welfare costs of pathologies in financial markets, including Stein (1987), DeLong, Shleifer, Summers, and Waldmann (1989), Chauvin, Laibson, and Mollerstrom (2011), and Lansing (2012). A large literature in macroeconomics and in corporate finance focuses on the sensitivity of firms' investment to a given mispricing in the stock market (Blanchard, Rhee, and Summers, 1993; Baker, Stein, and Wurgler, 2003; Gilchrist, Himmelberg, and Huberman, 2005; Farhi and Panageas, 2006). ${ }^{6}$ One conclusion from this literature is that the sensitivity of capital investment with respect to stock prices is low or that the stock market is a "sideshow" with respect to the real economy (Morck, Shleifer, and Vishny, 1990). By contrast, we show quantitatively that the social cost of a failure of information aggregation, transmitting itself through the channels outlined

investment opportunities, and Albagli (2011), where noise trader risk is amplified due to liquidity constraints on traders.

${ }^{3}$ The notion of near-rationality is due to Akerlof and Yellen (1985) and Mankiw (1985). Our application is closest to Cochrane (1989) and Chetty (2012), who use the utility cost of small deviations around an optimal policy to derive "economic standard errors." Other recent applications include Woodford (2010) and Dupor (2005).

${ }^{4}$ For an approach to pathologies in social learning based on social dynamics rather than on information externalities, see Burnside et al. (2011).

${ }^{5}$ The provision of public information thus always raises welfare in our framework (see Appendix A.11.1).

${ }^{6}$ Also see Galeotti and Schiantarelli (1994), Polk and Sapienza (2009), Panageas (2005), and Chirinko and Schaller (2006). 
above, is economically large even when this sensitivity is low. ${ }^{7}$

The quantitative application of our model also makes contact with an emerging literature that strives to document and explain stylized facts concerning the dynamics and dispersion of expectations (Patton and Timmermann, 2010; Yu, 2011; Coibion and Gorodnichenko, 2012; Sockin, 2015).

Finally, we add to the existing literature by quantifying the effects of our information microstructure within a full-fledged dynamic stochastic general equilibrium model. Existing models with dispersed information typically require policy functions that are (log) linear in the expectation of the shocks agents learn about (e.g., Hellwig, 2005; Lorenzoni, 2009; Angeletos, Lorenzoni, and Pavan, 2012; and Angeletos and La'O, 2013). By contrast, in standard RBC models with capital accumulation and decreasing returns to scale, households' policies are non-linear functions of the average expectation of future productivity. We are able solve and estimate our model due to recent advances in computational economics. We follow the solution method in Mertens (2009), which builds on Judd (1998) and Judd and Guu (2001) in using an asymptotically valid higher-order expansion in all state variables around the deterministic steady state of the model in combination with a nonlinear change of variables (Judd, 2002).

The remainder of the paper is structured as follows. Section 1 derives the main theoretical insights of the model in a simple three-period model. Section 2 introduces our mechanism into a quantitative RBC model with endogenous capital accumulation. Section 3 estimates the model and presents quantitative results.

\section{$1 \quad$ Static Model}

The model economy exists at three time periods $t=0,1,2$. At $t=1$, an endowment of a numéraire good can be stored until $t=2$, or converted into units of capital $K$ at adjustment $\operatorname{cost} \frac{1}{2 \kappa} K^{2}$, where $\kappa \geq 0$. At $t=2$, each unit of capital returns $\eta$ units of the numéraire:

$$
Y=\eta K, \eta \sim N\left(\bar{\eta}, \sigma_{\eta}^{2}\right)
$$

The capital adjustment technology is operated by an investment goods sector that performs instant arbitrage between the price of capital traded in a Walrasian stock market at $t=1, Q$, and the number of units of capital in circulation:

$$
\max _{K} \Pi=Q K-K-\frac{1}{2 \kappa} K^{2}
$$

\footnotetext{
${ }^{7}$ This finding also relates to a large literature on the costs of business cycles. See Barlevy (2005) for a survey.
} 
Its first-order condition requires that it supplies

$$
K=\kappa(Q-1)
$$

units of capital to maximize arbitrage profits.

A continuum of identical households indexed by $i \in[0,1]$ populates the economy. At $t=1$, each household observes $Q$ and receives a private signal about productivity

$$
s_{i}=\eta+\nu_{i},
$$

where $\nu_{i}$ represents i.i.d. draws from a normal distribution with zero mean and variance $\sigma_{\nu}^{2} .{ }^{8}$ Given $s_{i}$, each household chooses to purchase $z_{i}$ units of capital ("stocks") to maximize expected utility from terminal wealth, $\mathcal{E}_{1 i}\left[U_{i}\right]$, where

$$
U_{i}=w_{2 i}-\frac{\rho}{2} V_{1}\left[w_{2 i}\right]
$$

with risk aversion $\rho>0 . V_{1}\left[w_{2 i}\right]$ is the posterior variance given $s_{i}$ and $Q$, and terminal wealth is given by

$$
w_{2 i}=z_{i}(\eta-Q)+\Pi,
$$

where $\Pi$ is a lump-sum transfer of profits from the investment goods sector.

When forming their expectation about productivity, households make a small error. This small error shifts the posterior probability density function of $\eta$ by $\mu_{i}\left(\epsilon+\hat{\epsilon}_{i}\right)$, where $\mu_{i} \geq 0$ measures household $i$ 's exposure to the error $\epsilon+\hat{\epsilon}_{i}$. We refer to a household as near-rational if $\mu_{i}>0$ and as fully rational if $\mu_{i}=0$. The expectational error is positively correlated across households, where $\epsilon \sim N\left(0, \sigma_{\epsilon}\right)$ is the common component that is the same across all households, $\hat{\epsilon}_{i} \sim N\left(0, \hat{\mu} \sigma_{\epsilon}\right)$ is the idiosyncratic component, and $\hat{\mu}$ calibrates the size of the correlation across households. The expectations operator $\mathcal{E}_{1 i}$ is thus the rational expectations operator, except that it allows households to make small mistakes about the conditional mean of $\eta$ :

$$
\mathcal{E}_{1 i}[\eta]=E_{1 i}[\eta]+\mu_{i}\left(\epsilon+\hat{\epsilon}_{i}\right)
$$

where $E_{1 i}[\eta]=E\left[\eta \mid Q, s_{i}\right]$ is the rational expectations operator conditional on the information available to household $i$ at time 1. Although near-rational households make correlated mistakes when forming their expectations, they understand the structure of the economy, understand the equilibrium mapping of information into $Q$, and have the correct perception of all higher

\footnotetext{
${ }^{8}$ In section 1.4, we show that the conclusions of our model continue to hold in more general information environments where the noise in the private signal is correlated across households and where households observe an exogenous public signal in addition to their private signal.
} 
moments of the conditional distribution of $\eta \cdot{ }^{9}$ Importantly, households know they and others load on the common error, $\epsilon$.

There are many reasons why households may make small correlated errors in practice. For concreteness, we assume households choose to load on the near-rational error with $\mu_{i}>0$ because they face a small mental cost $\lambda|\bar{U}|\left(1-\mu_{i}\right)$ of behaving fully rationally, where $\bar{U}$ is households' certainty-equivalent wealth in the case in which all households behave fully rationally (i.e., when $\left.\mu_{i}=0 \forall i\right)$. The parameter $\lambda \ll 1$ calibrates this small mental cost of fully rational behavior as a share of certainty-equivalent wealth. For convenience, we normalize $\sigma_{\epsilon}$ such that

$$
\mu_{i}=\arg \max E_{0}\left[U_{i}\right]-\lambda|\bar{U}|\left(1-\mu_{i}\right)=1 \quad \forall i
$$

in the symmetric equilibrium, and thus

$$
\mathcal{E}_{1 i}[\eta]=E\left[\eta \mid Q, s_{i}\right]+\epsilon+\hat{\epsilon}_{i}
$$

One can show that $\sigma_{\epsilon}$ is a monotonic, strictly increasing function of $\lambda$ (see Appendix A.3 for a proof). That is, the larger the mental cost of behaving fully rationally, the larger the nearrational errors households make when forming their expectations. As a result, all comparative statics over $\lambda$ and $\sigma_{\epsilon}$ are qualitatively identical, and we use them interchangeably below. In the limit in which $\lambda \rightarrow 0$, all households behave fully rationally with $\sigma_{\epsilon} \rightarrow 0$.

Lastly, market clearing requires that aggregate demand for stocks is equal to the number of units of capital in circulation:

$$
\int_{0}^{1} z_{i} d i=K
$$

\subsection{Solving the Model}

Plugging (4) into (3) and taking the derivative with respect to $z_{i}$ yields households' optimal demand for stocks:

$$
z_{i}=\frac{\mathcal{E}_{1 i}[\eta]-Q}{\rho V_{1}[\eta]}
$$

We can then use the market-clearing condition (8) and plug in (9) and (1) to show the market price of capital is a linear function of the average expectation of $\eta$ :

$$
Q=\frac{\int_{0}^{1} \mathcal{E}_{1 i}[\eta] d i+\kappa \rho V_{1}[\eta]}{1+\kappa \rho V_{1}[\eta]}
$$

\footnotetext{
${ }^{9}$ Formally,
}

$$
\mathcal{E}_{1 i}\left[\left(\eta-\mathcal{E}_{1 i}(\eta)\right)^{k}\right]=E_{1 i}\left[\left(\eta-E_{1 i}(\eta)\right)^{k}\right] \forall k>1
$$


Households can thus directly infer the average expectation $\int \mathcal{E}_{1 i}[\eta] d i$ from observing $Q$. We may guess that the equilibrium price function is linear in $\eta$ and $\epsilon$ :

$$
Q=\pi_{0}+\pi_{1} \eta+\gamma \epsilon
$$

Assuming this guess is correct, we can write the rational expectation of $\eta$ given the private signal and $Q$ as

$$
E_{1 i}[\eta]=\alpha_{0}+\alpha_{1} s_{i}+\alpha_{2} \int_{0}^{1} \mathcal{E}_{1 i}[\eta] d i,
$$

where the constants $\alpha_{0}, \alpha_{1}$, and $\alpha_{2}$ are the weights households give to the prior, the private signal, and the average expectation, respectively. Using (7), substituting (2), and taking the integral across individuals gives

$$
\int_{0}^{1} \mathcal{E}_{1 i}[\eta] d i=\frac{\alpha_{0}}{1-\alpha_{2}}+\frac{\alpha_{1}}{1-\alpha_{2}} \eta+\frac{1}{1-\alpha_{2}} \epsilon
$$

where the noise in private signals, $\nu_{i}$, as well as the idiosyncratic errors, $\hat{\epsilon}_{i}$, integrate to zero. Plugging this expression back into (10) and matching coefficients with (11) verifies that the equilibrium price function is indeed linear.

It follows that in addition to $s_{i}$, households can extract an independent and unbiased signal about $\eta, \frac{\left(1-\alpha_{2}\right)}{\alpha_{1}} \int_{0}^{1} \mathcal{E}_{1 i}[\eta] d i-\frac{\alpha_{0}}{\alpha_{1}}=\eta+\frac{1}{\alpha_{1}} \epsilon$, from observing $Q$. Bayes' rule then implies the posterior variance is the inverse of the sum of the precision of the prior and the two signals:

$$
V\left[\eta \mid s_{i}, Q\right]=V_{1}[\eta]=\left(\sigma_{\eta}^{-2}+\sigma_{\nu}^{-2}+\alpha_{1}^{2} \sigma_{\epsilon}^{-2}\right)^{-1}
$$

Moreover, the conditional expectation of $\eta$ is the precision-weighted sum of the signals and the prior mean divided by the posterior precision ${ }^{10}$ :

$$
E\left[\eta \mid s_{i}, Q\right]=\frac{\sigma_{\eta}^{-2} \bar{\eta}+\sigma_{\nu}^{-2} s_{i}+\alpha_{1}^{2} \sigma_{\epsilon}^{-2}\left(\eta+\frac{1}{\alpha_{1}} \epsilon\right)}{V_{1}[\eta]^{-1}} .
$$

Matching coefficients with (12) yields

$$
\alpha_{0}=\frac{\bar{\eta} V_{1}(\eta) \sigma_{\epsilon}^{2}}{\sigma_{\eta}^{2}\left(\alpha_{1} V_{1}(\eta)+\sigma_{\epsilon}^{2}\right)}, \quad(16) \quad \alpha_{1}=\frac{V_{1}[\eta]}{\sigma_{\nu}^{2}}, \quad(17) \quad \alpha_{2}=\frac{\alpha_{1} V_{1}(\eta)}{\alpha_{1} V_{1}(\eta)+\sigma_{\epsilon}^{2}}
$$

Solving the system of equations consisting of the matched coefficients in (11), (14), (16), (17), and (18) leads to expressions in terms of parameters. Because the closed-form solution for $V_{1}[\eta]$ is somewhat cumbersome, it is convenient to rewrite the solution of all endogenous variables as a function of $V_{1}[\eta]$ and parameters.

\footnotetext{
${ }^{10}$ See Appendix A.1 for a more detailed proof.
} 


\section{Lemma 1.1}

The unique linear symmetric equilibrium of this economy is characterized as follows:

$$
\pi_{0}=\frac{V_{1}[\eta]\left(\bar{\eta}+\kappa \rho \sigma_{\eta}^{2}\right)}{\left(1+\kappa \rho V_{1}[\eta]\right) \sigma_{\eta}^{2}}, \quad(19) \quad \pi_{1}=\frac{\sigma_{\eta}^{2}-V_{1}[\eta]}{\left(1+\kappa \rho V_{1}[\eta]\right) \sigma_{\eta}^{2}}, \quad \text { (20) } \quad \gamma=\frac{\left(\sigma_{\eta}^{2}-V_{1}[\eta]\right) \sigma_{\nu}^{2}}{V_{1}[\eta]\left(1+\kappa \rho V_{1}[\eta]\right) \sigma_{\eta}^{2}}
$$

where the conditional variance is implicitly defined by

$$
V_{1}[\eta]=\left(\sigma_{\eta}^{-2}+\sigma_{\nu}^{-2}+\frac{V_{1}[\eta]^{2}}{\sigma_{\nu}^{4}} \sigma_{\epsilon}^{-2}\right)^{-1}
$$

Proof See Appendix A.2.

These results have two immediate implications. First, note the standard deviation of the idiosyncratic component of expectational errors, $\hat{\mu}$, appears in none of the solutions above and is thus irrelevant for the stock price, the conditional variance of $\eta$, and all aggregate variables. Second, although the sensitivity of the capital stock with respect to the stock price, $\kappa$, influences the response of the stock price to $\eta$ and $\epsilon$, it does not affect households' ability to learn about $\eta$ (the conditional variance of $\eta$ in (22) is independent of $\kappa$ ). Similarly, plugging (17) into (15) shows the loadings of households' expectation operators on $\eta$ and $\epsilon$ depend only on $\sigma_{\eta}, \sigma_{\nu}$, and $\sigma_{\epsilon}$, and not on $\kappa$. How much households can learn about the future is thus unrelated to the function linking stock prices to the real economy. We will show in section 2 that this insight carries over to a quantitative model in which the equilibrium expectations operator continues to be a function of the same three variables, despite much more complex and non-linear macroeconomic dynamics.

\subsection{External Effect on Information Aggregation}

The last two terms on the right-hand side of (13) reflect two channels through which nearrational behavior affects equilibrium expectations. The last term shows the small common error is amplified with the multiplier $1 /\left(1-\alpha_{2}\right)$. Because $\alpha_{2}$ is a number between 0 and 1 , the multiplier is always larger than 1, reflecting the fact that the stock price also transmits the common error whenever it transmits information. The extent of amplification depends on how much households (rationally) rely on $Q$ when forming their expectation of $\eta$. The bigger the weight they place on the stock price, $\alpha_{2}$, the larger the amplified noise in equilibrium expectations relative to $\epsilon$. The second term on the right-hand side reflects the indirect effect of near-rational behavior, which arises due to the fact that households optimally calculate the coefficients $\alpha_{1}$ and $\alpha_{2}$. When the market price of capital transmits an amplified common error in addition to information about $\eta$, households rationally lower $\alpha_{2}$ and, as a result, decrease the equilibrium information content of stock prices. 
To understand these two effects, it is useful to consider the limiting case in which all households are fully rational, which arises when the mental cost of fully rational behavior, and thus the size of near-rational errors, goes to zero $(\lambda \rightarrow 0)$. In this case, the model coincides with a standard rational expectations equilibrium in which the stock price is perfectly revealing about future productivity (commonly referred to as the "Grossman equilibrium"). Appendix A.4 shows that

$$
\lim _{\lambda \rightarrow 0}\left[V_{1}[\eta]\right]=0
$$

and

$$
\lim _{\lambda \rightarrow 0}\left[\pi_{1}\right]=1 .
$$

In this case, households put all weight on the stock price $\left(\alpha_{2}=1\right)$ and no weight on their private signal $\left(\alpha_{1}=0\right)$. In this limit, a marginally small common error is infinitely amplified, $\gamma=1 /\left(1-\alpha_{2}\right)=\infty$. The direct effect of near-rational behavior is thus to generate large nonfundamental errors in the market price of capital and in equilibrium expectations. The indirect effect of near-rational behavior is households' rational reaction to this fact. When the stock price transmits amplified common errors, households rationally reduce $\alpha_{2}$, such that the multiplier on $\epsilon$ becomes finite. However, a reduction of $\alpha_{2}$ also reduces the elasticity of the stock price with respect to information, $\pi_{1}$. The following proposition formalizes this intuition.

\section{Proposition 1.2}

Near-rational behavior globally decreases the elasticity of the stock price with respect to future productivity:

$$
\frac{\partial \pi_{1}}{\partial \lambda}<0 \quad \forall \lambda>0 .
$$

As the mental cost of fully rational behavior (and thus $\sigma_{\epsilon}$ ) approaches 0 , the marginal effect of near-rational behavior on this elasticity becomes infinitely large:

$$
\lim _{\lambda \rightarrow 0}\left[\frac{\partial \pi_{1}}{\partial \lambda}\right]=-\infty
$$

Proof See Appendix A.5.

The result of this proposition can alternatively be restated in terms of the size of the errors: $\partial \pi_{1} / \partial \sigma_{\epsilon} \rightarrow-\infty$ for $\sigma_{\epsilon} \rightarrow 0$. (We find an infinite, rather than just a large, multiplier at the margin because the stock price becomes perfectly revealing of all available information when $\lambda \rightarrow 0$. If some other exogenous source of noisy demand remained even when $\lambda=0$, this amplification would instead be finite.)

It follows that near-rational behavior has a first-order detrimental effect on the stock market's capacity to transmit information. This result contrasts sharply with the utility considerations of an individual household that loads on the small common error. By the envelope theorem, the 
first-order effect from loading on (small) near-rational errors is zero:

$$
\lim _{\lambda \rightarrow 0} \frac{\partial E_{0}\left[U_{i}\right]}{\partial \mu_{i}}=0
$$

Both the demand schedule for stocks in (9) and the expectations operator (12) are the result of an optimization. Near-rational errors, even if they contain a common component, represent a small deviation from the household's optimal program. Because the slope of the utility function at the optimum is 0 , the marginal effect of these deviations on utility is also 0 .

The key to understanding this result is that a hypothetical rational household $\left(\mu_{i}=0\right)$ that lives in an economy populated by near-rational households $\left(\mu_{j \neq i}=1\right)$ has no informational advantage. Near-rational households understand fully that they and other households are making errors when forming their expectations. They react to this fact by lowering $\alpha_{2}$ in response to a rise in the equilibrium amount of non-fundamental volatility in the stock price. Because learning about $\eta$ is isomorphic to learning about $\epsilon$, near-rational households thus already do everything possible to learn about the common component of the near-rational error. ${ }^{11}$ Absent an informational advantage, the optimal behavior of a fully rational household is then simply to implement the same optimal program, but without the near-rational error. The utility gain from behaving fully rationally instead of near rationally is thus 0 at the margin.

By continuity, proposition 1.2 implies that for small $\sigma_{\epsilon}$, near-rational behavior represents an externality that has a first-order detrimental effect on the market's ability to aggregate information, whereas households have only a negligible (lower-order) incentive to avoid making these errors. The stock market thus fails to aggregate information even though it is efficient in the sense that a fully rational household cannot systematically outperform a near-rational household with the same information set.

We next consider the comparative static of this result when private information becomes more dispersed in the economy.

\section{Proposition 1.3}

1. The more dispersed private information is, the more detrimental the effect of near-rational errors on information aggregation:

$$
\frac{\partial \pi_{1}}{\partial \sigma_{\nu}}=-\frac{\left(1+\kappa \rho \sigma_{\eta}^{2}\right)\left(2 \sigma_{\nu}^{2} \sigma_{\epsilon}^{2} V_{1}[\eta]^{2}+4 V_{1}[\eta]^{4}\right)}{\sigma_{\eta}^{2}\left(1+\kappa \rho V_{1}[\eta]\right)^{2}\left(\sigma_{\nu}^{5} \sigma_{\epsilon}^{2}+2 \sigma_{\nu} V_{1}[\eta]^{3}\right)} \begin{cases}<0 & \text { if } \lambda>0 \\ =0 & \text { in the limit } \lambda \rightarrow 0 .\end{cases}
$$

2. Any strictly positive mental cost of fully rational behavior may destroy the stock market's

\footnotetext{
${ }^{11}$ From (11), knowing $\eta$ and $Q$ is clearly the same as knowing $\epsilon$ and $Q$; we could thus rewrite the entire optimal program using a signal extraction about $\epsilon$ and obtain the same result.
} 


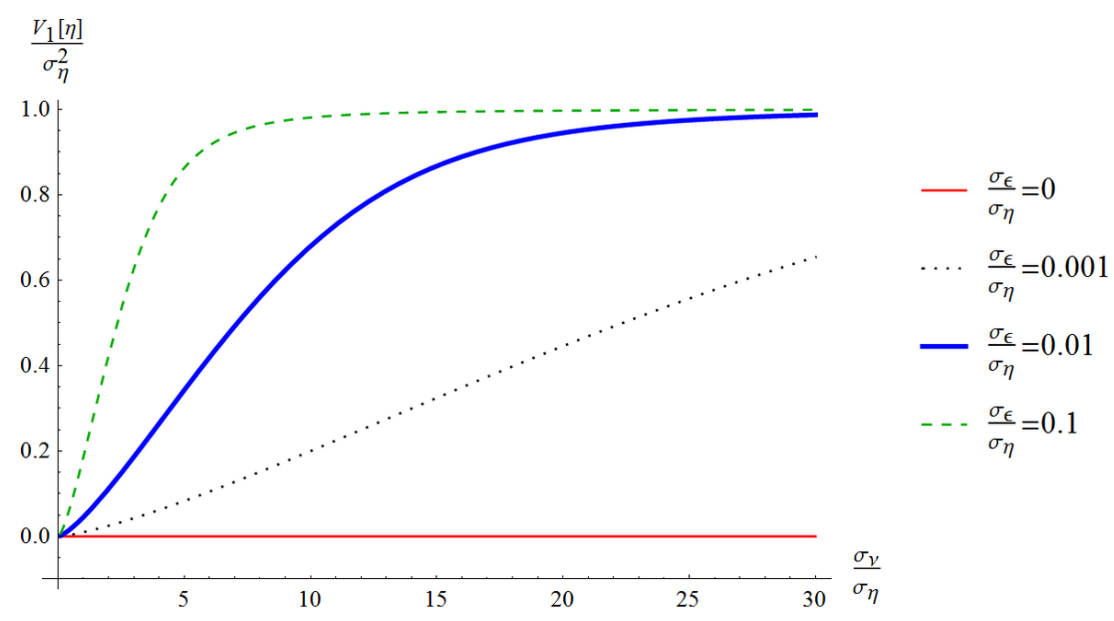

Figure 1: Ratio of the conditional variance of $\eta$ to its unconditional variance plotted over the level of dispersion of private information, $\sigma_{\nu} / \sigma_{\eta}$.

capacity to aggregate information as the dispersion of private information goes to infinity:

$$
\lim _{\sigma_{\nu} \rightarrow \infty}\left[\frac{V_{1}[\eta]}{\sigma_{\eta}^{2}}\right]= \begin{cases}1 & \text { if } \lambda>0 \\ 0 & \text { in the limit } \lambda \rightarrow 0 .\end{cases}
$$

Proof See Appendix A.6.

Figure 1 illustrates this point. It plots the ratio of the conditional variance of $\eta$ to its unconditional variance over the level of dispersion of private information. To facilitate the interpretation of the results, we scale all standard deviations with the standard deviation of the productivity shock, $\sigma_{\eta}$. With this scaling, all standard deviations have a natural interpretation. In particular, the ratio $\left(\frac{\sigma_{\nu}}{\sigma_{\eta}}\right)^{2}$ measures the level of dispersion of information in the economy as the number of individuals who, in the absence of a market price, would need to pool their private information in order to reduce the conditional variance of $\eta$ by one half. A value of 0 on the vertical axis indicates households can perfectly predict tomorrow's realization of $\eta$, whereas a value of 1 indicates $\eta$ is completely unpredictable.

The horizontal red line shows that when all households behave fully rationally $\left(\lambda, \sigma_{\epsilon}=0\right)$, $\eta$ is perfectly predictable, regardless of how dispersed information is in the economy. In this case, the stock price perfectly transmits all available information in the economy. This situation changes drastically when $\lambda, \sigma_{\epsilon}>0$. The thick blue line plots the results for the case in which the standard deviation of the common component of the near-rational error is $1 \%$ of the standard deviation of $\eta$. The curve rises steeply and converges to 1 . To the very left of the graph, when the private signal is more precise and households thus rely relatively little on the stock price when learning about the future, near-rational behavior has a relatively small detrimental effect 
on information aggregation. However, when we move to the right of the graph, households rely more on the stock price, and near-rational behavior has a larger detrimental effect. When private information is highly dispersed, near-rational behavior results in the total collapse of information aggregation. This result is quite general and continues to hold in more complex settings. In fact, our quantitative model in section 2 will produce a figure almost identical to Figure 1.

The second statement in Proposition 1.3 implies this qualitative result does not depend on how near-rational households are. For comparison, Figure 1 plots the comparative statics for near-rational errors that are an order of magnitude larger $\left(\frac{\sigma_{\epsilon}}{\sigma_{\eta}}=0.1\right)$ and an order of magnitude smaller $\left(\frac{\sigma_{\epsilon}}{\sigma_{\eta}}=0.001\right)$. In each case, the productivity shock becomes completely unpredictable if information is sufficiently dispersed.

The implication of this finding is that information aggregation in financial markets is most likely to break down precisely when it is most socially valuable - when information is highly dispersed. This comparative static again contrasts sharply with the incentives an individual household faces:

\section{Proposition 1.4}

Holding $\lambda$ fixed, households make larger near-rational errors the more dispersed information is;

$$
\frac{\partial \sigma_{\epsilon}}{\partial \sigma_{\nu}}>0
$$

\section{Proof See Appendix A.\%.}

For a given mental cost of fully rational behavior, households make larger near-rational errors precisely when they are more socially harmful. In other words, making mistakes of a given magnitude $\sigma_{\epsilon}$ becomes cheaper as we move from left to right in Figure 1. The reason is, again, that learning about $\epsilon$ is isomorphic to learning about $\eta$. When households' private signals are more precise, they know more about $\eta$ and thus more easily detect the common component of the near-rational error, which makes near-rational behavior costlier (it pays to be fully rational if you can identify and trade against $\epsilon$ ). When private signals are noisier and information aggregation breaks down, no one can learn much about the future. The less households can learn about the future, the less they can distinguish movements in stock prices that are due to $\eta$ from movements that are due to $\epsilon$. Making near-rational errors is thus cheaper precisely when they are most socially harmful.

Due to this additional amplification, the deterioration in the information content of stock prices when increasing $\sigma_{\nu}$ is more pronounced when we hold fixed $\lambda$ than if we hold fixed $\sigma_{\epsilon}$, as illustrated in Appendix Figure 1.

A crucial feature of these results is again that any collapse in the aggregation of information 


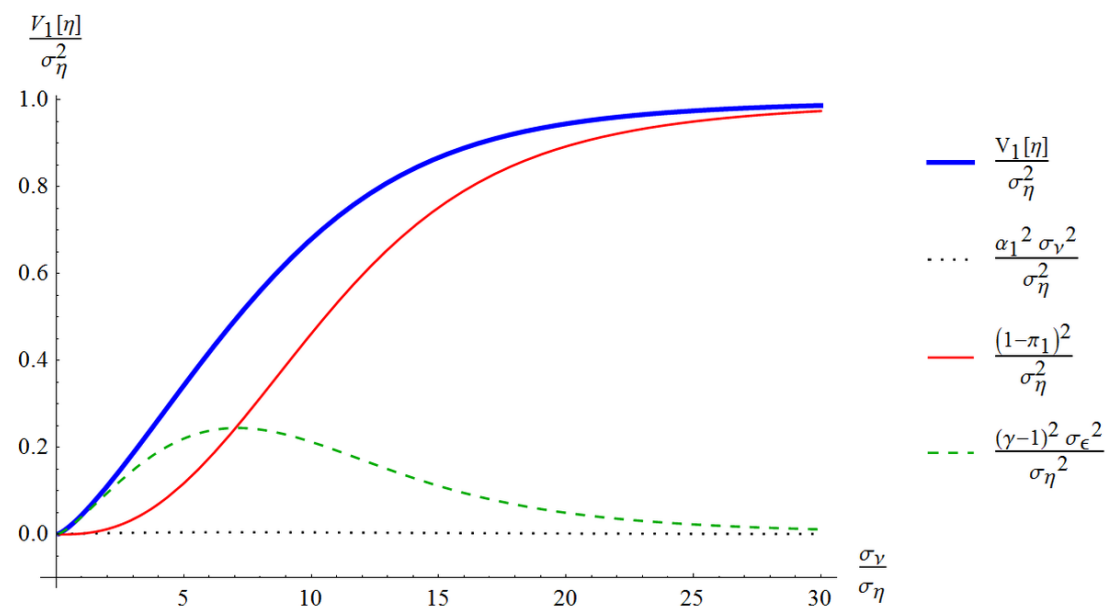

Figure 2: Decomposition of the ratio of the conditional variance of $\eta$ to its unconditional variance plotted over the level of dispersion of private information.

affects everyone in the economy: conditional on receiving the same private signal, the difference in the expectation of a rational and near-rational household is small, $\epsilon+\hat{\epsilon}_{i}$. In fact, the posterior variance we plotted in Figure 1 is the conditional variance of such a fully rational household:

$$
\frac{V_{1}[\eta]}{\sigma_{\eta}^{2}}=\frac{1}{\sigma_{\eta}^{2}}\left(\alpha_{1}^{2} \sigma_{\nu}^{2}+\left(1-\pi_{1}\right)^{2} \sigma_{\eta}^{2}+(\gamma-1)^{2} \sigma_{\epsilon}^{2}\right)
$$

The expression for the precision of the forecast of a near-rational household is identical, except the third term in brackets is then $\gamma^{2} \sigma_{\epsilon}^{2}{ }^{12}$

Figure 2 decomposes the conditional variance (27) into its three components. The thick blue line in Figure 2 is the same as the thick blue in Figure 1. It plots the ratio of the conditional variance of $\eta$ to its unconditional variance over the level of dispersion of private information for the case in which $\frac{\sigma_{\epsilon}}{\sigma_{\eta}}=0.01$. The dotted line plots the first term on the right-hand side of (27), which is the error households make in their forecast of $\eta_{t+1}$ due to the noise in their private signal. It is roughly constant throughout, reflecting the fact that households reduce $\alpha_{1}$ when the private signal contains more noise. The broken line plots the second term, which is the error households make in their forecast because the stock price does not reflect all information about $\eta_{t+1}$ (the "indirect" effect of near-rational behavior), and the third component is the error households make due to amplified common errors in the stock price (the "direct" effect of near-rational behavior).

At low levels of $\sigma_{\nu}$, amplified small common errors are the main source of households' forecast

\footnotetext{
${ }^{12}$ This result follows from the analysis in Appendix A.1. By "precision" of the forecasts of near-rational household, we refer to $\frac{E_{i t}\left[\left(\eta_{t+1}-\mathcal{E}_{i t}\left(\eta_{t+1}\right)\right)^{2}\right]}{\sigma_{\eta}^{2}}$. Note, however, that from (6), the near-rational household has the same "perceived" conditional variance as a rational household, $\mathcal{E}_{i t}\left[\left(\eta_{t+1}-\mathcal{E}_{i t}\left(\eta_{t+1}\right)\right)^{2}\right]=E_{i t}\left[\left(\eta_{t+1}-E_{i t}\left(\eta_{t+1}\right)\right)^{2}\right]$.
} 
errors. As private information becomes more dispersed, the amplification rises and eventually peaks as households, confronted with noisy private signals and a noisy stock price, begin to rely more on their priors, reducing both $\alpha_{1}$ and $\alpha_{2}$. At the same time, the information content of the stock price begins to fall. In the region in which the broken line approaches 1, small common errors result in a complete collapse of information aggregation, and $\alpha_{1}, \alpha_{2} \rightarrow 0$.

\subsection{Real Effects of Near-rational Behavior}

Now that we understand the aggregation of information in our model, we can ask how nearrational behavior affects the economy as a whole. Using (11), we can write equilibrium stock returns as

$$
\eta-Q=-\pi_{0}+\eta\left(1-\pi_{1}\right)-\gamma \epsilon
$$

Near-rational behavior affects each of the three terms on the right-hand side. The third term on the right-hand side shows that it induces non-fundamental volatility in stock returns. Equation (25) shows that near-rational behavior reduces $\pi_{1}$ and thus causes a rise in the volatility of equilibrium stock returns (in the sense that the volatility of stock returns is 0 when all households behave fully rationally, and positive if and only if $\lambda, \sigma_{\epsilon}>0$ ). Near-rational behavior thus results in an increase in the amount of financial risk households face in the stock market. Lastly, this increase in financial risk in turn induces a rise in the equity premium by lowering the first term. Taking time-zero expectations of (28) and using (19) and (20) to substitute for $\pi_{0}$ yields

$$
E_{0}[\eta-Q]=\kappa \rho(\bar{\eta}-1)\left(\frac{\left(1-\pi_{1}\right) \sigma_{\eta}^{2}}{1+\kappa \rho \sigma_{\eta}^{2}}\right) .
$$

These effects on stock returns are mirrored in distortions of aggregate quantities:

\section{Proposition 1.5}

Near-rational behavior lowers the covariance between capital accumulation and productivity and reduces the expected level of capital accumulation and output by impeding the stock market's capacity to aggregate information:

$$
\frac{\partial E_{0}[K]}{\partial \lambda}=\frac{\partial E_{0}[K]}{\partial \pi_{1}} \frac{\partial \pi_{1}}{\partial \lambda}<0 \text { and } \frac{\partial \operatorname{Cov}_{0}[K, \eta]}{\partial \lambda}=\frac{\partial \operatorname{Cov}_{0}[K, \eta]}{\partial \pi_{1}} \frac{\partial \pi_{1}}{\partial \lambda}<0 .
$$

Proof See Appendix A.8.

Our model allows us to assess the welfare effects of these distortions while avoiding two common difficulties in noisy rational expectations models (e.g., Brunnermeier et al. (2014)) First, because the model features only a single class of agents, we can calculate the ex-ante (time zero) utility of these agents without having to consider the utility of non-maximizing "noise" or "liquidity" traders that are commonly used as a modeling device to induce noise 
in the equilibrium stock price. Second, because near-rational households are, by definition, "nearly" maximizing their utility, whether we consider welfare under the fully rational or the near-rational measure is inconsequential. Because the biases in the behavior of near-rational households are small, whether we respect them for the purposes of our utility calculations does not matter; that is, $\mathcal{E}_{0}\left[U_{i}\right] \approx E_{0}\left[U_{i}\right]$. Using these two insights, we can show the following lemma:

Lemma 1.6

The ex-ante utilitarian social welfare function can be written as

$$
S W F=E_{0}\left[U_{i}\right]=E_{0}\left[w_{2 a}\right]-\frac{\rho}{2} E_{0}\left[V_{1}\left[w_{2 i}\right]\right]
$$

where

$$
E_{0}\left[V_{1}\left[w_{2 i}\right]\right]=V_{0}\left[w_{2 a}\right]+V_{0}\left[w_{2 i}-w_{2 a}\right]-V_{0}\left[E_{1 i}\left[w_{2 i}\right]\right]
$$

and

$$
E_{0}\left[w_{2 a}\right]=(\bar{\eta}-1) E_{0}[K]+\operatorname{Cov}_{0}(K, \eta)-\frac{1}{2 \kappa} E_{0}\left[K^{2}\right]
$$

Proof See Appendix A.9.

The utilitarian social welfare function thus depends on the unconditional mean and variance

of aggregate wealth, $w_{2 a} \equiv K(\eta-Q)+\frac{K^{2}}{2 \kappa}$, the expected dispersion of wealth across households (reflecting the fact that cross-sectional dispersion of consumption represents a source of risk from the perspective of the household), and the amount of information about $w_{2 i}$ revealed at $t=1$ (reflecting a preference for early resolution of uncertainty).

\section{Proposition 1.7}

Near-rational behavior lowers welfare by lowering the level of aggregate wealth, increasing the dispersion of wealth across households, and delaying the resolution of uncertainty. These effects represent a negative externality of near-rational behavior that transmits itself through the effect of near-rational errors on the stock market's capacity to transmit information:

$$
\lim _{\lambda \rightarrow 0}\left[\frac{\partial S W F}{\partial \lambda}-\frac{\partial E_{0}\left[U_{i}\right]}{\partial \mu_{i}}\right]=\lim _{\lambda \rightarrow 0}\left[\frac{\partial S W F}{\partial \pi_{1}} \frac{\partial \pi_{1}}{\partial \lambda}\right]=\sigma_{\eta}^{2} \frac{\sigma_{\nu}^{2} \rho \kappa\left(1+\kappa \rho\left((1-\bar{\eta})^{2}+\sigma_{\eta}^{2}\right)\right)+1}{2 \sigma_{\nu}^{2} \rho\left(1+\kappa \rho \sigma_{\eta}^{2}\right)} \lim _{\lambda \rightarrow 0}\left[\frac{\partial \pi_{1}}{\partial \lambda}\right]<0 .
$$

Proof See Appendix A.10.

By reducing the market's capacity to transmit information, near-rational behavior thus has a large (first-order) external effect on welfare. This effect works through three channels. First, it lowers aggregate wealth by distorting the aggregate capital stock, output, and consumption. Second, it increases the dispersion of wealth across households, because households differ in their equilibrium expectations and investments when information aggregation breaks down. Third, 
it delays the resolution of uncertainty, because households know less about the future in equilibrium. Taken together, near-rational behavior thus entails first-order social costs while it is costless to the individual household at the margin. In the following section, we use our quantitative model to gauge the overall size of these costs in the data and the relative contributions of the three channels above.

Note, however, that even these qualitative results have implications beyond their immediate application to the present paper: a large literature on the real effects of stock market dysfunctionality has traditionally focused on estimating either the sensitivity of capital investment with respect to stock prices, $\kappa$, or the covariance of capital investment with non-fundamental movements of stock prices, $\operatorname{Cov}_{0}(K, \epsilon)$. Our results above show that neither of these quantities are a sufficient statistic of the welfare consequences of near-rational behavior (instead, they are at best partially informative only about the first channel). In this sense, our results show the social costs of a dysfunctional stock market can be large even if the stock market appears as a "sideshow" (Morck, Shleifer, and Vishny, 1990) in the sense that $\kappa$ is low.

\subsection{Alternative Information Environments}

The key result of the analysis above is that small correlated errors in household expectations have a lower-order effect on an individual household's utility but a first-order external effect on social welfare. We now show this result carries over to richer information environments in which households are exposed to other noisy signals. We consider two alternative sources of correlated errors in expectations.

In the first specification, households can additionally observe a noisy public signal about $\eta$,

$$
g=\eta+\varpi
$$

where $\varpi \sim N\left(0, \sigma_{\varpi}^{2}\right)$. In the second specification, we introduce aggregate noise in private signals of the form

$$
s_{i}=\eta+\nu_{i}+\zeta
$$

where $\zeta \sim N\left(0, \sigma_{\zeta}^{2}\right)$, such that the integral over private signals is no longer perfectly revealing about $\eta$.

\section{Proposition 1.8}

If there exists either a noisy public signal (as in (31)) or aggregate noise in the private signal (as in (32)), the marginal effect of near-rational behavior on the elasticity of the stock price with respect to productivity continues to become infinitely large as the mental cost of fully rational 
behavior (and thus $\sigma_{\epsilon}$ ) approaches zero:

$$
\lim _{\lambda \rightarrow 0}\left[\frac{\partial \pi_{1}}{\partial \lambda}\right]=\lim _{\sigma_{\epsilon} \rightarrow 0}\left[\frac{\partial \pi_{1}}{\partial \sigma_{\epsilon}}\right]=-\infty
$$

By contrast, the marginal effects of noise in the public signal and aggregate noise in the private signal on this elasticity go to 0 as their standard deviations go to 0 :

$$
\lim _{\sigma_{\varpi} \rightarrow 0}\left[\frac{\partial \pi_{1}}{\partial \sigma_{\varpi}}\right]=0 \quad \text { and } \quad \lim _{\sigma_{\zeta} \rightarrow 0}\left[\frac{\partial \pi_{1}}{\partial \sigma_{\zeta}}\right]=0, \quad \text { respectively } .
$$

Proof See Appendix A.11.

This proposition has two direct implications. First, it demonstrates that none of our results rely on the stock price becoming perfectly revealing of $\eta$ if all households are fully rational. Near-rational behavior continues to have a first-order effect on information aggregation even if aggregate noise is present in the private signal or if households observe other (exogenous) public signals aside from the stock price.

Second, near-rational errors $(\epsilon)$ are amplified in equilibrium while noise introduced through the signal structure $(\varpi$ and $\zeta$ ) is not. To see the intuition for this result, consider the equilibrium price function in a general model that allows for these other types of noisy signals (shown for $\kappa=0)$ :

$$
Q=\frac{\alpha_{0}}{1-\alpha_{2}}+\frac{\alpha_{1}+\alpha_{3}}{1-\alpha_{2}} \eta+\frac{\alpha_{1}}{1-\alpha_{2}} \zeta+\frac{\alpha_{3}}{1-\alpha_{2}} \varpi+\frac{1}{1-\alpha_{2}} \epsilon
$$

where $\alpha_{3}$ is the weight households put on the exogenous public signal when forming expectations. The result now follows from the fact that $\frac{\alpha_{1}+\alpha_{3}}{1-\alpha_{2}}=\pi_{1}, \frac{\alpha_{1}}{1-\alpha_{2}}$, and $\frac{\alpha_{3}}{1-\alpha_{2}}$ are always numbers between 0 and 1, whereas the coefficient on near-rational errors $\frac{1}{1-\alpha_{2}}$ ranges between 1 and $\infty$. Near-rational errors are amplified in equilibrium because they affect the market's capacity to aggregate information. By contrast, noise introduced through the signal structure merely affects the amount of information that is available for aggregation, but does not result in the kind of externality shown in Proposition 1.7.

Figure 3 illustrates these findings. The thick blue line plots the now familiar effect of a small common error in household expectations with $\frac{\sigma_{\epsilon}}{\sigma_{\eta}}=0.01$. The red horizontal line plots the effect of an identical amount of small aggregate noise in the private signal (i.e., $\frac{\sigma_{\zeta}}{\sigma_{\eta}}=0.01$ ). The red line has an intercept of $0.01^{2}$ and is perfectly horizontal. Aggregate noise in the private signal is not amplified, and the fact that an individual household observes a signal with common noise does not have an external effect on the market's capacity to aggregate information. The effect of aggregate noise in the private signal is thus invariant to how dispersed information is in the economy. The broken lines in Figure 3 show the same comparative static, but in the presence of large aggregate noise in the private signal $\left(\frac{\sigma_{\zeta}}{\sigma_{\eta}}=1\right)$. Both lines retain their shape but now 


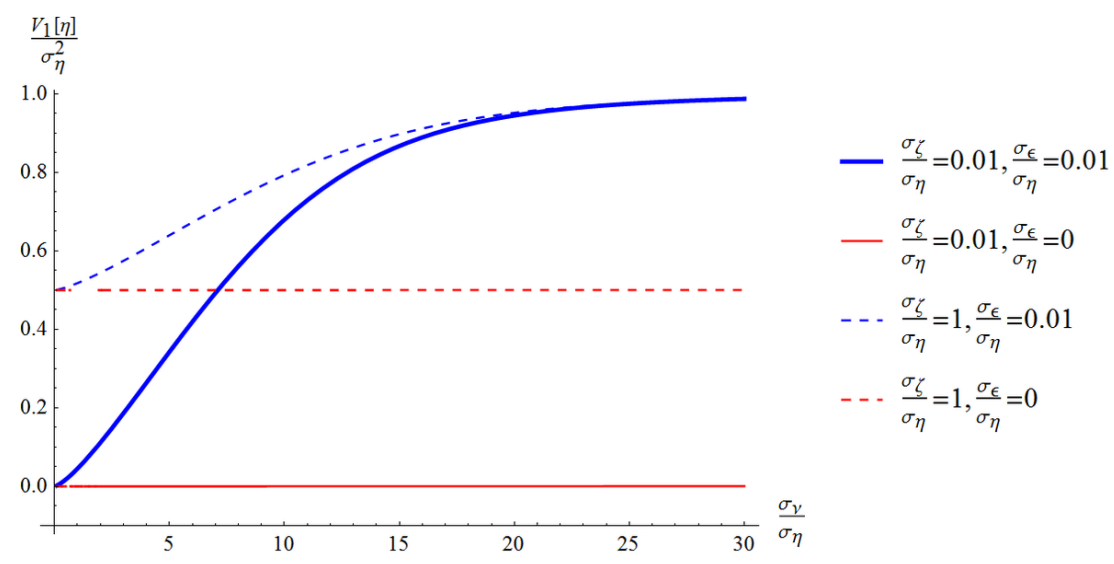

Figure 3: Comparison of the effects of small common errors in household expectations with the effects of small common noise in the private signal (solid lines) and large common noise in the private signal (broken lines).

have a higher intercept, reflecting the fact that less information is now available to aggregate, even if the stock price is fully revealing. However, for the remaining dispersed information, the information externality of near-rational behavior operates in the same way as in Figure 1. The externality is thus relevant whenever dispersed private information exists that is valuable for predicting $\eta$. A key challenge for our quantitative analysis below will thus be to estimate the degree of dispersion in private information in the data.

The appendix contains several additional results. Appendix A.12 contrasts our near-rational approach with the model of Hellwig (1980). In this model, the market's capacity to aggregate information depends on the volatility of exogenous noise trader demand, where a separate class of agents ("noise traders") inelastically purchases random quantities of stocks. In Appendix A.13, we also consider near-rational errors about the second conditional moment (the conditional variance of $\eta$ ), which we show to be isomorphic to the near-rational errors about the conditional expectation in (7) under some additional assumptions. In Appendix A.14, we extend our environment to allow rational households to obtain an informational advantage over near-rational households by observing the error they would have made had they acted near-rationally. We show that even using this alternative counterfactual, all the main results of our analysis go through, provided the standard deviation of idiosyncratic errors, $\hat{\mu}$, is sufficiently large.

\section{Quantitative Model}

In this section, we set up a quantitative model that allows us to estimate the welfare effects and the equilibrium impact of near-rational behavior. To this end, we use a decentralization of the RBC model by Croce (2014). We choose this model for two reasons. First, it performs 
well in matching both macroeconomic and asset-pricing moments and should therefore provide a reliable quantitative assessment of the macroeconomic effects of asset market dysfunctionality. Second, it assumes households have recursive preferences as in Epstein and Zin (1989) that directly price news and therefore represent a natural choice in the context of our analysis where near-rational behavior distorts news about the future.

\subsection{Setup}

Technology is characterized by a linear homogeneous production function that uses capital, $K_{t}$, and labor, $N_{t}$, as inputs:

$$
Y_{t}=K_{t}^{\alpha}\left(e^{a_{t}} N_{t}\right)^{1-\alpha}
$$

where $Y_{t}$ stands for output of the consumption good. The productivity of labor, $a_{t}$, has a long-run component, $\omega$, and a short-run component, $\eta^{S}$ :

$$
\Delta a_{t+1}=\mu_{a}+\omega_{t}+\eta_{t+1}^{S}
$$

where the long-run component follows

$$
\omega_{t}=\rho \omega_{t-1}+\eta_{t}^{L}
$$

Both shocks to productivity, $\eta^{S}$ and $\eta^{L}$, are i.i.d. normally distributed with mean zero and standard deviations $\sigma_{\eta S}$ and $\sigma_{\eta L}$, respectively.

The equation of motion of the capital stock is

$$
K_{t+1}=\left(1-\delta_{k}\right) K_{t}+I_{t}-G_{t} K_{t}
$$

where $I_{t}$ denotes aggregate investment and $\delta_{k}$ is the rate of depreciation. Furthermore, there are convex adjustment costs to capital following Jermann (1998):

$$
G_{t}=\frac{I_{t}}{K_{t}}-\left(\frac{v_{1}}{1-\frac{1}{\xi}}\left(\frac{I_{t}}{K_{t}}\right)^{1-\frac{1}{\xi}}+v_{0}\right)
$$

where $v_{1}$ and $v_{2}$ are positive constants and the parameter $\xi$ determines the equilibrium elasticity of the capital stock with respect to the stock price.

A representative firm purchases capital and labor services from households. Because it rents services from an existing capital stock, the firm's objective collapses to a period-by-period maximization problem:

$$
\max _{K_{t}, N_{t}} Y_{t}-d_{t} K_{t}-w_{t} N_{t}
$$


where $K_{t}$ and $N_{t}$ denote factor demands for capital and labor, respectively. First-order conditions with respect to capital and labor pin down the market-clearing wage, $w_{t}$, as the marginal product of labor and the rental rate of capital, $d_{t}$, as the marginal product of capital. Because the production function is linear homogeneous, the representative firm makes zero economic profits from producing the consumption good.

The representative firm also owns an investment goods sector that converts the consumption good into units of capital, while incurring adjustment costs. It takes the price of capital as given and then performs instantaneous arbitrage:

$$
\max _{I_{t}} Q_{t}\left(I_{t}-G_{t} K_{t}\right)-I_{t}
$$

Taking the first-order condition of this profit function gives us the equilibrium price of capital (Tobin's Q), $Q_{t}=1 / 1-G_{t}^{\prime}$. Because of decreasing returns to scale in converting consumption goods to capital, the investment goods sector makes positive profits in each period. Profits are paid to shareholders as a part of dividends per share, $D_{t}=\alpha \frac{Y_{t}}{K_{t}}+Q_{t}\left(G_{t}^{\prime} \frac{I_{t}}{K_{t}}-G_{t}\right) \cdot{ }^{13}$

A continuum of households on the interval $i \in[0,1]$ has Epstein and Zin (1989) preferences over the consumption bundle $\tilde{C}_{i t}$ :

$$
U_{i t}=\left((1-\delta) \tilde{C}_{i t}^{1-\frac{1}{\psi}}-\pi\left(b_{i t}\right)+\delta \mathcal{E}_{i t}\left[U_{i t+1}^{1-\gamma}\right]^{\frac{1-\frac{1}{\psi}}{1-\gamma}}\right)^{\frac{1}{1-\frac{1}{\psi}}}
$$

where the parameters $\psi$ and $\gamma$ measure the households' intertemporal elasticity of substitution and relative risk aversion, respectively. $\pi\left(b_{i t}\right)$ is a small penalty for holding bonds that ensures a well-defined portfolio choice at the deterministic steady state (Judd and Guu, 2001). ${ }^{14}$ The consumption bundle $\tilde{C}_{i t}$ is a Cobb-Douglas aggregate of consumption and leisure:

$$
\tilde{C}_{i t}=C_{i t}^{o}\left(e^{a_{t-1}}\left(1-n_{i t}\right)\right)^{1-o}
$$

where leisure scales with aggregate productivity to ensure the existence of a balanced growth path.

At the beginning of every period, each household receives a private signal about the shocks to long-run and short-run productivity:

$$
s_{i t}^{j}=\eta_{t+1}^{j}+\nu_{i t}^{j}, j=L, S,
$$

\footnotetext{
${ }^{13}$ Alternatively, profits may be paid to households as a lump-sum transfer; this assumption matters little for the quantitative results of the model.

${ }^{14}$ We use the simple quadratic form $\pi\left(b_{i t}\right)=\frac{1}{2000} e^{a_{t-1}\left(1-\frac{1}{\psi}\right)}\left(\frac{b_{i t}}{e^{a} t-1}\right)^{2}$.
} 
where $\nu_{i t}^{j}$ again represents i.i.d. draws from a normal distribution with zero mean and variance $\sigma_{\nu j}^{2}, j=L, S$. In some specifications, we also allow for aggregate noise in these private signals as in (32). To further distinguish the implications of near-rational behavior from the implications of noisy signals, we allow households to observe an exogenous noisy public signal about each of the two shocks,

$$
g_{t}^{j}=\eta_{t+1}^{j}+\varpi_{t}^{j}, j=L, S,
$$

with $\varpi^{j} \sim N\left(0, \sigma_{\varpi j}\right)$.

Households observe all prices and aggregate state variables at time $t$ and understand the structure of the economy as well as the equilibrium mapping of dispersed information into prices and economic aggregates. The rational expectations operator, conditional on all the information available to household $i$ at time $t$, is

$$
E_{i t}[\cdot]=E\left[\cdot \mid s_{i t}^{L}, s_{i t}^{S}, g_{t}^{L}, g_{t}^{S}, Q_{t}, r_{t}, d_{t}, w_{t}, C_{t}, N_{t}, K_{t}, Y_{t}, I_{t}, G_{t}, a_{t}, \omega_{t-1}\right]
$$

where $r_{t}$ is the risk-free interest rate. The only sources of uncertainty are thus the two innovations, $\eta_{t+1}^{L}$ and $\eta_{t+1}^{S}$, and households must form a conditional expectation of them. As in the model in section 1, we assume households make small common errors when forming these expectations. The expectations operator $\mathcal{E}_{i t}$ in (39) is thus the rational expectations operator with the only exception that

$$
\mathcal{E}_{i t}\left[\eta_{t+1}^{j}\right]=E_{i t}\left[\eta_{t+1}^{j}\right]+\mu_{i}^{j}\left(\epsilon_{t}^{j}+\hat{\epsilon}_{i t}^{j}\right)
$$

where again $\epsilon_{t}^{j} \sim N\left(0, \sigma_{\epsilon j}\right)$ and $\hat{\epsilon}_{i t}^{j} \sim N\left(0, \hat{\mu} \sigma_{\epsilon j}\right), j=L, S$. For simplicity, we assume the near-rational errors about the two innovations are uncorrelated with each other and i.i.d.

Given the private and public signals and their knowledge about the state of the economy, households maximize lifetime utility (39) by choosing a time path for consumption and labor, and their holdings of stocks and bonds $\left\{\tilde{C}_{i t}, n_{i t}, k_{i t}, b_{i t}\right\}_{t=0}^{\infty}$. Each household's optimization is subject to a budget constraint:

$$
Q_{t} k_{i t+1}+b_{i t}=Q_{t-1} R_{t} k_{i t}+\left(1+r_{t-1}\right) b_{i t-1}+H_{i t}-C_{i t}+w_{t} n_{i t},
$$

where $H_{i t}$ are transfers from state-contingent claims discussed below. The returns to capital are

$$
R_{t+1}=\frac{\left(1-\delta_{k}\right) Q_{t+1}+\alpha \frac{Y_{t+1}}{K_{t+1}}+Q_{t+1}\left(G_{t+1}^{\prime} \frac{I_{t+1}}{K_{t+1}}-G_{t+1}\right)}{Q_{t}} .
$$

The market-clearing conditions for the stock, bond, labor, and goods markets are 
$K_{t+1}=\int k_{i t+1} d i$

and

$$
0=\int b_{i t} d i
$$

$$
N_{t}=\int n_{i t} d i
$$

$$
Y_{t}=C_{t}+I_{t}
$$

Finally, the payments from contingent claims, $H_{i t}$, avoid having to keep track of the evolution of wealth across households. ${ }^{15}$ At the beginning of each period (and before receiving signals), households can trade claims that are contingent on the state of the economy and on the realization of the noise they receive in their private signals, $\nu_{i t}^{j}$. These claims are in zero net supply and pay off at the beginning of the next period. Because the claims are traded before any information about $\eta_{t+1}^{j}$ is known, their prices cannot reveal any information about future productivity. Contingent-claims trading thus completes markets between periods, without affecting households' signal-extraction problem. In equilibrium, all households choose to hold these securities with net payoff

$$
H_{i t}= \begin{cases}Q_{t-1} R_{t} K_{t}-Q_{t-1} R_{t} k_{i t}-\left(1+r_{t-1}\right) b_{i t-1} & \text { if }\left\{C_{i t}, n_{i t}, k_{i t}, b_{i t}\right\}=\left.\arg \max (39)\right|_{H_{i t}=0} \\ 0 & \text { otherwise }\end{cases}
$$

such that all households enter each period with the same amount of wealth. From (44) and (45), it follows immediately that these claims are in zero net supply, $\int H_{i t} d i=0$.

The rational expectations equilibrium is the economy in which $\mu_{i}^{j}=0 \forall i, j=L, S$, such that the expectations operator $\mathcal{E}_{i t}$ in equation (39) coincides with the rational expectation in (40). The near-rational expectations equilibrium posits that $\mu_{i}^{j}=1 \forall i$; households make small errors as given in (41). In Appendix B.2, we provide a formal definition of equilibrium and show the first-order and envelope conditions.

\subsection{Solving the model}

We use the solution method developed in Mertens (2009) to transform the equilibrium conditions of the model into a form we can solve with standard techniques. The key to this approach is to show that all prices and economic aggregates are a function of the usual "macroeconomic" state variables of the model $S_{t}=\left\{K_{t}, \omega_{t-1}, \eta_{t}^{L}, \eta_{t}^{S}\right\}$ as well as households' average expectation of $\eta_{t+1}^{L}$ and $\eta_{t+1}^{S}$ :

$$
\hat{q}_{t}^{j}=\int \mathcal{E}_{i t}\left[\eta_{t+1}^{j}\right] d i=\int E_{i t}\left[\eta_{t+1}^{j}\right] d i+\epsilon_{t}^{j} \quad \text { for } j=S, L
$$

\footnotetext{
${ }^{15}$ See Mertens and Judd (2013) for a perturbation-based approach to solving incomplete market models with substantial heterogeneity.
} 


\section{Lemma 2.1}

A recursive equilibrium exists with the following properties:

(1) A household's optimal behavior depends on the current (commonly known) state of the economy, $S_{t}$, the household's conditional expectation of next period's innovation to long-run and short-run productivity, $\mathcal{E}_{i t}\left[\eta_{t+1}^{j}\right]$, and the average expectation of this innovation, $\hat{q}_{t}^{j}, j=L, S$. The conditional expectation, in turn, depends on the private signal $s_{i t}^{j}$ as well as $\hat{q}_{t}^{j}$. Individual choices are thus determined by these state variables:

$$
x_{i t}=x\left(S_{t}, \hat{q}_{t}^{L}, \hat{q}_{t}^{S}, \mathcal{E}_{i t}\left[\eta_{t+1}^{L}\right], \mathcal{E}_{i t}\left[\eta_{t+1}^{S}\right]\right), x=C, n, k_{+1}, b .
$$

(2) All prices and economic aggregates depend on the current state of the economy and $\hat{q}_{t}^{j}$ :

$$
X_{t}=X\left(S_{t}, \hat{q}_{t}^{L}, \hat{q}_{t}^{S}\right), X=C, N, K_{+1}, Y, I, G, R, Q, r, d, w
$$

Proof: See Appendix B.3.

Given this lemma, we can use standard perturbation methods to solve for households' equilibrium policies as a function of the vector $\left\{S_{t}, \hat{q}_{t}^{L}, \hat{q}_{t}^{S}, \mathcal{E}_{i t}\left[\eta_{t+1}^{L}\right], \mathcal{E}_{i t}\left[\eta_{t+1}^{S}\right]\right\}$ and for all economic aggregates as a function of $\left\{S_{t}, \hat{q}_{t}^{L}, \hat{q}_{t}^{S}\right\}$. In other words, we can separate the solution of the non-linear model from the information microstructure by simply treating $\mathcal{E}_{i t}\left[\eta_{t+1}^{j}\right]$ and $\hat{q}_{t}^{j}$ as state variables. The final step of the solution is then to solve for $\mathcal{E}_{i t}\left[\eta_{t+1}^{j}\right]$.

\section{Condition 2.2}

A pair of aggregate quantities and/or prices exists that is invertible in $\hat{q}^{L}$ and $\hat{q}^{S}$, conditional on knowing all other state variables.

This condition ensures two observable variables (e.g., the returns on stocks and bonds) exist that are strictly monotonic in average expectation about $\eta^{L}$ and $\eta^{S}$ but have different sensitivities, such that households can infer $\hat{q}^{S}$ and $\hat{q}^{L}$ from observing the two variables conditional on the state of the economy. Learning from these variables is then just as good as directly observing $\hat{q}^{S}$ and $\hat{q}^{L}$ (they span the same $\sigma$-algebra). Although we cannot solve for the mapping of $\hat{q}^{S}$ and $\hat{q}^{L}$ into prices and aggregate quantities in closed form, we verify invertibility using the numerical solution of the model.

Typically, many combinations of aggregate variables and prices meet these criteria. That is, because households know the state of the economy, they have many ways of inferring $\hat{q}^{S}$ and $\hat{q}^{L}$, for example, by observing output and wages. In this sense, asset prices are not special and we have no way to distinguish learning from asset prices from learning from other prices or aggregate variables. For ease of exposition, we nevertheless follow the existing literature and refer to this process as learning from asset prices, because asset prices may be more easily and 
frequently observable in practice - however keeping in mind that our results do not speak to the question whether households in reality learn from asset prices versus other economic aggregates.

Given Lemma 2.1 and Condition 2.2, households' equilibrium expectations of $\eta_{t+1}^{j}$ for $j=$ $S, L$ are independent of the aggregate dynamics of the model. Due to the normality of conditioning variables $s_{i t}^{j}$ and $\hat{q}_{t}^{j}$ for $j=S, L$ respectively, the resulting conditional distributions are Gaussian and identical to the linear setup in section 1. As a result, all the qualitative results concerning the effect of the near-rational errors on equilibrium expectations developed in our static model readily carry over to the quantitative model. Appendix B.4 contains a formal treatment of these statements.

\section{Estimation and Results}

We next quantify the implications of our model by taking it to the data, estimating the size of near-rational errors, the dispersion in private signals, and the precision of public signals.

\subsection{Estimation strategy}

To focus our efforts on the novel part of the model, its information microstructure, and to preserve a maximum of comparability with earlier work, we set all parameters not directly related to information equal to the benchmark calibration proposed by Croce (2014). Table 1 lists these parameters. For example, the capital share, $\alpha$, is set to $0.345, \delta_{k}$ is set to match the annualized capital depreciation rate in the US economy $(6 \%)$, and the average annual growth rate of productivity, $\mu_{a}$, is $1.8 \%$. Relative risk aversion and the intertemporal elasticity of substitution are set to values of 10 and 2 , respectively, and the annualized subjective discount factor, $\delta$, is fixed at 0.954 . Crucial for the model's capacity to match asset prices, the annualized persistence of the long-run shock to productivity is 0.8 , while the standard deviation of the long-run shock is relatively small ( $11 \%$ of the standard deviation of the short-run shock). The parameters $v_{1}$ and $v_{2}$ in the adjustment-cost function are set such that, at the deterministic steady state, $G_{t}=0$ and $\partial G_{t} / \partial\left(I_{t} / K_{t}\right)=0$ (implying that $v_{0}=\left(\frac{1}{1-\xi}\right)\left(\delta+e^{\mu}-1\right)$ and $\left.v_{1}=\left(\delta+e^{\mu}-1\right)^{\frac{1}{\xi}}\right)$.

We also follow Croce (2014) in calculating excess stock returns as the excess returns on a levered claim to capital that is subject to idiosyncratic cash-flow shocks: $R_{e x, t}^{L E V}=\left(R_{t}-r_{t-1}+u_{t}\right)^{\phi_{l e v}}$. This practice is standard in the finance literature because, in the data, payouts to equity are more volatile than consumption and most claims to equity are levered. We again follow Croce (2014) by setting the annualized standard deviation of $u$ to $6.5 \%$ (consistent with Bansal and Yaron (2004)) and $\phi_{l e v}=2$ (consistent with the amount of financial leverage measured by Rauh and Sufi (2012)). 
Table 1: Calibrated Parameters

\begin{tabular}{lcc}
\hline \hline Parameter & & Value \\
\hline Capital share & $\alpha$ & 0.345 \\
Time discount factor & $\delta^{12}$ & 0.954 \\
Avg. productivity growth & $12 \mu_{a}$ & 0.018 \\
Consumption share & $o$ & 0.20 \\
Autocorrelation of $\eta^{L}$ & $\rho^{12}$ & 0.8 \\
Ratio of standard dev. of $\eta^{L}$ and $\eta^{S}$ & $\sigma_{\eta L} / \sigma_{\eta S}$ & 0.11 \\
Capital depreciation rate & $12 \delta_{k}$ & 0.06 \\
Relative risk aversion & $\gamma$ & 10 \\
Intertemporal elasticity of substitution & $\psi$ & 2 \\
Leverage of market return & $\phi_{\text {lev }}$ & 2.0 \\
Capital adjustment cost parameter & $\xi$ & 7 \\
\hline \hline
\end{tabular}

Notes: Calibrated parameters taken from the benchmark calibration in Croce (2014) and used in all specifications shown in Tables 2 to 5.

We also maintain the standard practice in the long-run risk literature by using a monthly decision horizon and then aggregating the model-implied macroeconomic and financial time series to the annual frequency to match annual moments (Bansal and Yaron, 2004; Bansal et al., 2012). However, note this choice of decision horizon is less innocuous in our case, because the decision horizon also determines the horizon at which households learn about the future. That is, in our main specification, households make decisions at the monthly frequency and receive signals about the realization of productivity shocks one month (rather than a quarter or a year) in the future. For this reason, we measure empirical moments disciplining the dynamics of expectations and the amount of information available to households at the same horizon as in the model (one month ahead), rather than at the annual frequency. In robustness checks, we also show our main results do not change when we instead calibrate the model at the quarterly frequency and thus allow households to observe signals about shocks one quarter ahead.

For parsimony, we set the standard deviation of the idiosyncratic component of near-rational errors to $0(\hat{\mu}=0)$ and estimate the standard deviation of short-run productivity shock, $\sigma_{\eta S}$, as well as the six parameters governing the information microstructure $\left(\sigma_{\nu j}, \sigma_{\epsilon j}, \sigma_{\varpi j}, j=S, L\right)$, using the simulated method of moments. Our estimator of these parameters, $\hat{m}$, minimizes the distance between a vector of empirical moments, $\theta$, and the corresponding moments generated by the model, $m$, subject to the constraint that our estimates of $\sigma_{\epsilon}^{j}$ are small enough such that the utility gain of behaving fully rather than near-rationally is economically small:

$$
\hat{m}=\arg \min _{m}(m-\theta)^{\prime} \Omega(m-\theta) \text { s.t. } \lambda(m)<\bar{\lambda},
$$

where $\Omega$ is our estimate of the variance-covariance matrix of the empirical moments, calculated 
using a bootstrap procedure detailed in Appendix C.1, $\lambda$ is the compensating variation in terms of permanent consumption for individual $i$ of setting $\mu_{i}=0$ in all future periods, and $\bar{\lambda}$ is a measure from the data of the minimum size of incentives required to induce households to engage in fully rational behavior. For a given parameter vector, we create 100 synthetic time series, each of length equal to our sample size. Following the procedure in Albuquerque, Eichenbaum, and Rebelo (2015), we then calculate $m$ as the probability limit of the model-implied small-sample moments by calculating the sample moments of interest from each sample and then averaging across samples.

Similar to the private cost of near-rational behavior, $\lambda$, we measure the social cost of nearrational behavior as the compensating variation for individual $i$ of setting $\mu_{j}=0 \forall j \neq i$. Both calculations are performed at the stochastic steady state of the model, such that our measure for the social cost of near-rational behavior takes into account that the abolition of near-rational behavior today induces an adjustment process to a new stochastic steady state. See Appendix C.2 for details on this calculation.

\subsection{Data}

Our target vector, $\theta$, contains 17 empirical moments listed in the first column of Table 3. Because our focus is on identifying the information microstructure of the model, the first five of these targets are of particular importance - characterizing the dynamics and dispersion of expectations using data from the Survey of Professional Forecasters 1969-2008. All of our calculations use forecasts for the current quarter, that is, forecasts of quarterly GDP and consumption growth made six weeks before the end of the quarter, which is the horizon closest to the one-monthahead forecasts households make in our model. The first two targets measure the degree of uncertainty about future GDP growth: the variance of the average forecast of GDP growth and the variance of the average forecast error (both normalized with the standard deviation of GDP growth). The remaining three moments characterize the dispersion in expectations held by individual forecasters: the time-series average of the cross-sectional standard deviation of forecasts of GDP growth, the correlation of this dispersion with future consumption growth, and its monthly autocorrelation. Aside from quantifying the dispersion of expectations in the data, these latter two moments reflect two salient stylized facts about disagreement highlighted in the literature - that disagreement is counter-cyclical (Bloom, 2014; Patton and Timmermann, 2010), and that it is highly persistent at the monthly frequency (Yu, 2011). Appendix C.1 gives details on the construction of these moments. ${ }^{16}$

The remaining 12 entries in the target vector are standard macroeconomic and financial moments that are typically used to discipline long-run-risk models. They include the standard

\footnotetext{
${ }^{16}$ Related to these stylized facts, $\mathrm{Li}$ and $\mathrm{Li}$ (2014) find that turnover in the stock market increases with the dispersion of expectations, suggesting this dispersion is driven, at least in part, by heterogenous private information.
} 
deviation of output, the equity premium, and the correlation of stock returns with consumption growth. All of these moments are constructed from annual US data (1929-2008) in a manner identical to that used by Croce (2014) (see Appendix C.3 for details).

Although the calculation of these moments is fairly straightforward and has ample precedent in the literature, we are unaware of existing estimates of the minimum size of economic incentives required to induce households to engage in fully rational behavior, $\bar{\lambda}$. To nevertheless discipline our choice of this parameter, we follow three alternative approaches. In our benchmark estimation, we rely on the observation from the household finance literature that tests of rational consumption smoothing tend to reject only in natural experiments involving small stakes, whereas those involving large stakes tend to find evidence consistent with rational behavior. Following the procedure in Fuchs-Schuendeln and Hassan (2015), and using the parameters $\gamma$ and $\psi$ from Table 1, we calculate the utility gain as a percent of permanent consumption from smoothing a pre-announced income change over a year versus behaving as a hand-to-mouth consumer for each of 17 published studies on the subject (see Appendix C.4 for details). Our results, listed in Appendix Table 1, show that experiments corresponding to an equivalent variation of more than $0.13 \%$ of consumption on average find evidence consistent with rational behavior, whereas those involving smaller stakes do not. In the extremes, all experiments involving stakes smaller than $0.01 \%$ of consumption reject rational behavior, whereas none of those with stakes larger than $0.37 \%$ do. We thus use $\bar{\lambda}=0.13 \%$ in our benchmark calibration. In robustness checks, we explore the entire range $0.01 \%$ to $0.37 \%$, use alternative estimates from the rational inattention literature (Maćkowiak and Wiederholt, 2015), and also estimate $\lambda$ without a constraint.

\subsection{Results}

We present the results of our estimation in the following order. First, we discuss our findings regarding the information microstructure and relate them to the insights developed in our static model. We then show that the model gives a good fit to the data, discuss the effects of nearrational behavior on the economy, and show how these effects empirically identify the information microstructure. Lastly, we probe the robustness of our results.

We first discuss and interpret the benchmark estimates in the first column of Table 2-the best fit to the data according to (50). The estimated dispersion of private information about the long-run and short-run shocks are $\sigma_{\nu}^{L} / \sigma_{\eta}^{L}=23.59$ and $\sigma_{\nu}^{S} / \sigma_{\eta}^{S}=13.16$, respectively, implying that in the absence of other sources of information, $23.59^{2}=556$ and $13.6^{2}=185$ households would have to pool their private signals about the two shocks to reduce their conditional variance by one half. The standard deviation of the common error in household expectations of the long-run shock is $0.09 \%$ of the standard deviation of the shock to long-run productivity, but is amplified by a factor of 537, such that a one-standard-deviation shock to $\epsilon^{L}$ results in a $0.48 \sigma_{\eta L}$ rise in 
the average expectation of $\eta^{L}$ in equilibrium. Similarly, the standard deviation of the common error about the short-run shock is $0.42 \%$ of the standard deviation of the shock to short-run productivity, but is amplified by a factor of 99 .

As a result of this amplified noise, we find that equilibrium asset prices transmit about as much noise as information. The noise-to-signal ratios are 0.97 and 1.28 about the longrun and short-run shock, respectively. Nevertheless, even these noisy asset prices transmit enough information to reduce the ratio of conditional to unconditional variance to 0.49 for the long-run shock and 0.56 for the short-run shock. By contrast, our estimates show that both exogenous public signals are relatively imprecise $\left(\sigma_{\varpi L} / \sigma_{\eta L}=3.69\right.$ and $\left.\sigma_{\varpi S} / \sigma_{\eta S}=1.99\right)$, such that a household observing only their private signal and the exogenous public signal would have corresponding ratios of 0.94 and $0.79 .{ }^{17}$ Thus, most of what households learn about the future they learn from dispersed private information that is aggregated in asset prices.

Next, we build intuition and link these quantitative estimates to the theoretical results from our static model. Figure 4 shows the familiar comparative static over the level of dispersion of private information, while holding constant all other benchmark estimates. The top panels again plot the ratio of the conditional to unconditional variance of $\eta^{L}$ and $\eta^{S}$. Both graphs show the same shape as Figure 1, but they asymptote to $\left(1+3.96^{-2}\right)^{-1}=0.94$ and $\left(1+1.99^{-2}\right)^{-1}=0.80$ respectively, reflecting the fact that households can extract a small amount of information from the exogenous public signals even when the market's capacity to aggregate information collapses completely. Apart from this modification, a given $\sigma_{\epsilon S}$ or $\sigma_{\epsilon L}$ again has a larger effect on the stock market's capacity to aggregate information when private information is more dispersed, where the distance between the solid blue line and the red dashed line shows the external effect of near-rational behavior on the average expectation held by market participants.

Equipped with these estimates of the amount of information and amplified noise contained in asset prices, we calculate that a given household would be willing to pay $5.31 \%$ of its permanent consumption for all other households to forgo their near-rational behavior and behave fully rationally until the end of time. The social cost of near-rational behavior is thus an order of magnitude larger than the private cost $(0.13 \%$ of permanent consumption). The second row in Figure 4 shows the social cost generated by this externality is monotonically increasing in $\sigma_{\nu S}$ and $\sigma_{\nu L}$, ranging up to $9.84 \%$ of consumption when all private information about $\eta^{L}$ is driven out of prices $\left(\sigma_{\nu L} \rightarrow \infty\right)$. The social value of the part of information aggregation that does not get crowded out by near-rational behavior is thus equivalent to $9.84 \%-5.13=4.53 \%$ of permanent consumption. By contrast, near-rational errors about $\eta^{S}$ appear to have a much smaller impact on welfare (we return to this observation below). Although the social cost of near-rational behavior is economically large and monotonically increasing in $\sigma_{\nu S}$ and $\sigma_{\nu L}$, the

\footnotetext{
${ }^{17}$ Using equation (14) and the numbers given in Table 2 , we get $\left(1+23.59^{-2}+3.96^{-2}\right)^{-1}=0.94$ and $(1+$ $\left.13.16^{-2}+1.99^{-2}\right)^{-1}=0.79$.
} 
Table 2: Estimation Results

\begin{tabular}{|c|c|c|}
\hline & $\begin{array}{c}\text { Benchmark Model } \\
\text { (Noisy Public Signal) }\end{array}$ & $\begin{array}{l}\text { Aggregate Noise } \\
\text { in Private Signal }\end{array}$ \\
\hline \multicolumn{3}{|l|}{ Panel A: Parameter Estimates } \\
\hline \multicolumn{3}{|l|}{ Near-Rational Error } \\
\hline$\sigma_{\epsilon \mathrm{L}} / \sigma_{\eta \mathrm{L}}(\%)$ & 0.09 & 0.09 \\
\hline$\sigma_{\epsilon \mathrm{S}} / \sigma_{\eta \mathrm{S}}(\%)$ & 0.42 & 0.35 \\
\hline \multicolumn{3}{|l|}{ Noise in Private Signal } \\
\hline$\sigma_{\nu \mathrm{L}} / \sigma_{\eta \mathrm{L}}$ & 23.59 & 24.04 \\
\hline$\sigma_{\nu \mathrm{S}} / \sigma_{\eta \mathrm{S}}$ & 13.61 & 13.71 \\
\hline \multicolumn{3}{|l|}{ Std.dev. of productivity } \\
\hline$\sigma_{\eta \mathrm{S}}(\%)$ & 0.98 & 0.98 \\
\hline \multicolumn{3}{|l|}{ Noise in Public Signal } \\
\hline$\sigma_{\varpi \mathrm{L}} / \sigma_{\eta \mathrm{L}}$ & 3.69 & \\
\hline$\sigma_{\varpi \mathrm{S}} / \sigma_{\eta \mathrm{S}}$ & 1.99 & \\
\hline \multicolumn{3}{|c|}{ Aggregate Noise in Private Signal } \\
\hline$\sigma_{\zeta \mathrm{L}} / \sigma_{\eta \mathrm{L}}$ & & 0.00 \\
\hline$\sigma_{\zeta \mathrm{S}} / \sigma_{\eta \mathrm{S}}$ & & 0.11 \\
\hline \multicolumn{3}{|c|}{ Panel B: Implications for Information Aggregation } \\
\hline \multicolumn{3}{|l|}{ Conditional Variance } \\
\hline$V_{t}[\eta L] / \sigma_{\eta L}^{2}$ & 0.49 & 0.50 \\
\hline$V_{t}[\eta S] / \sigma_{\eta S}^{2}$ & 0.56 & 0.57 \\
\hline \multicolumn{3}{|c|}{ Amplification of near-rational error $\left(1 /\left(1-\alpha_{2}\right)\right)$} \\
\hline multiplier on $\epsilon^{L}$ & 536.75 & 577.86 \\
\hline multiplier on $\epsilon^{S}$ & 99.02 & 142.99 \\
\hline \multicolumn{3}{|c|}{ Noise-to-signal ratios in asset prices } \\
\hline Noise-to-signal about $\eta^{L}$ & 0.97 & 1.00 \\
\hline Noise-to-signal about $\eta^{S}$ & 1.28 & 1.32 \\
\hline \multicolumn{3}{|c|}{ Panel C: Implications for Welfare } \\
\hline Private Cost & 0.13 & 0.13 \\
\hline Social Cost & 5.31 & 5.40 \\
\hline
\end{tabular}

Notes: Panel A shows estimated parameters using the simulated method of moments. The target and simulated moments of the estimation are shown in column 1 of Table 3. The simulated moments are shown in columns 2 and 6, respectively. Panels $\mathrm{B}$ and $\mathrm{C}$ show the implications of these estimates for information aggregation and welfare. The social cost of near-rational behavior is the compensating variation for an intervention that commits all other households to behave fully rationally until the end of time $\left(\mu_{j}=0 \forall j \neq i\right.$.), calculated at the stochastic steady state of the model. Similarly, the private cost of near-rational behavior is the compensating variation for eliminating the near-rational error only from the household's own behavior. 

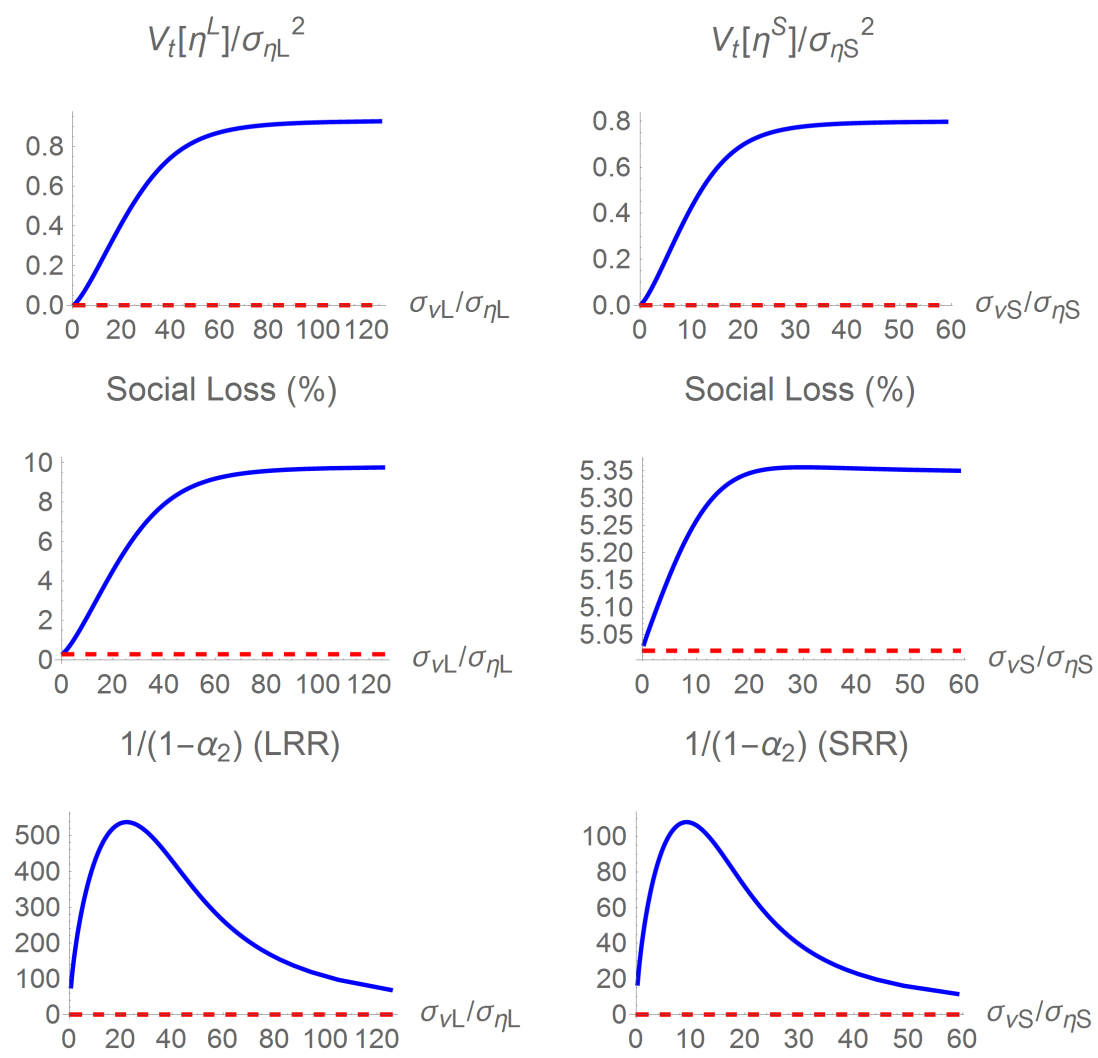

Figure 4: Comparative statics over dispersion of private information about the long-run (left column) and short-run (right column) shock to TFP growth $\left(\sigma_{\nu L}\right.$ and $\left.\sigma_{\nu S}\right)$, holding constant all other parameters of the benchmark estimation given in Tables 1 and 2. The red dashed line shows the results corresponding to full rationality $\left(\sigma_{\epsilon L}=\sigma_{\epsilon S}=0\right)$. First row: ratio of conditional to unconditional variance of the shock; second row: social cost of near-rational behavior in percent of permanent consumption; third row: multiplier on near-rational error in average expectation about the shock.

private cost is smaller than $0.13 \%$ of permanent consumption over the entire range (not shown).

The last row of Figure 4 shows the multiplier on the near-rational error, $1 /\left(1-\alpha_{2}\right)$. Consistent with Figure 2 in section 1, it shows the amplification peaks around a factor of 550 and 120 for intermediate values of $\sigma_{\nu L}$ and $\sigma_{\nu S}$, respectively. The estimates from our benchmark model are slightly to the right of the peak in both cases. As we move farther to the right in the graph, the noise-to-signal ratio in asset prices increases to the point that households give up learning from prices, begin to lower both $\alpha_{1}$ and $\alpha_{2}$, and instead rely on their priors. Eventually, the coefficients $\alpha_{1}$ and $\alpha_{2}$ converge to zero, whereas the noise-to-signal ratio and the social cost of near-rational behavior continue to increase monotonically as more and more information is driven out of prices.

Next, we show that the model performs well in matching the dynamics of expectations 
measured in the data. Column 2 of Table 3 shows the simulated moments generated by our benchmark estimation. In particular, the model comes close to matching the standard deviation of the average expectation of GDP growth (0.58 vs. 0.64 in the data) and the average forecast error of GDP growth (0.54 vs. 0.69 in the data). It also matches both stylized facts about the dynamics of disagreement by generating a negative correlation between consumption growth and the cross-sectional standard deviation of expectations (-0.58 vs. -0.34 in the data), as well as high levels of persistence of this standard deviation (0.98 vs. 0.88 in the data).

The crucial feature enabling our model to match both of these stylized facts is the combination of the i.i.d. Gaussian information microstructure and the non-linear dynamics of our RBC framework. A household's optimal consumption is a concave function of expected future productivity. A constant dispersion in expectations about productivity thus naturally results in higher levels of dispersion in expectations about consumption growth in a recession (when the consumption function is steep) than in a boom (when it is relatively flat). This dispersion in turn is highly persistent because of a high degree of persistence in the long-run shock and the level of the capital stock. Despite these successes, more disagreement exists among forecasters on average than our model can rationalize - it significantly under-predicts the average cross-sectional standard deviation of expectations (0.04 vs. 0.11 in the data). However, we may speculate that in reality forecasters also disagree for reasons outside our model, for example due to differences in priors (Burnside et al., 2011).

More broadly, the model also offers a good fit to the standard macro and asset-pricing moments shown in the remainder of Table 3, matching nine of 12 moments within two standard errors. However, it significantly under-predicts the correlation of consumption growth with investment ( 0.42 vs. 0.68 in the data), the standard deviation of the risk-free rate (1.28 vs. 3.82 in the data), and the standard deviation of stock returns (13.36 vs. 21.21 in the data). All three of these shortcomings are common in this type of long-run-risk model, and also apply to Croce (2014). Nevertheless, the introduction of near-rational behavior moves the model closer to the data in at least one of these dimensions, as we discuss below.

To highlight the role of near-rational behavior in generating these results, column 3 shows the simulated moments we obtain when imposing full rationality, that is, $\sigma_{\epsilon L}, \sigma_{\epsilon S}=0$, on our benchmark estimates. Comparing columns 2 and 3 shows three sets of effects of near-rational behavior on the economy, reminiscent of those we derived in our static model.

First, near-rational behavior generates a dispersion in equilibrium expectations: under full rationality, all households hold identical expectations in equilibrium because asset prices perfectly reveal all available information about $\eta_{t+1}^{L}$ and $\eta_{t+1}^{S}$, such that all three moments relating to the dispersion in expectations naturally degenerate.

This ability to explain disagreement among forecasters thus identifies the combined role of 
Table 3: Fit of Estimated Models

\begin{tabular}{|c|c|c|c|c|c|c|}
\hline Moments & $\overline{(1)}$ & $\overline{\overline{(2)}}$ & $\overline{\overline{(3)}}$ & $\overline{(4)}$ & $\overline{(5)}$ & $\overline{(6)}$ \\
\hline \multirow[t]{2}{*}{ Moments } & $\begin{array}{c}\text { Data } \\
\text { (std. err.) }\end{array}$ & & $\begin{array}{r}\text { Benchmar } \\
\text { (Noisy Publ }\end{array}$ & $\begin{array}{l}\text { Model } \\
\text { c Signal) }\end{array}$ & & $\begin{array}{l}\text { Aggregate Noise } \\
\text { in Private Signal }\end{array}$ \\
\hline & & & $\sigma_{\epsilon L}, \sigma_{\epsilon S}=0$ & $\sigma_{\epsilon L}=0$ & $\sigma_{\epsilon S}=0$ & \\
\hline$\sigma(\bar{E}[d y]) / \sigma(d y)$ & $\begin{array}{c}0.64 \\
(0.02)\end{array}$ & 0.58 & 0.96 & 0.57 & 0.98 & 0.57 \\
\hline$\sigma(d y-\bar{E}[d y]) / \sigma(d y)$ & $\begin{array}{c}0.69 \\
(0.08)\end{array}$ & 0.54 & 0.28 & 0.56 & 0.21 & 0.55 \\
\hline$\sigma_{x s}\left(E_{i}[d y]\right)(\%)$ & $\begin{array}{c}0.11 \\
(0.01)\end{array}$ & 0.04 & 0 & 0.04 & 0.01 & 0.04 \\
\hline $\operatorname{cor}\left(d c, \sigma_{x s}\left(E_{i}[d y]\right)\right)$ & $\begin{array}{l}-0.34 \\
(0.14)\end{array}$ & -0.58 & $N / A$ & -0.57 & 0.23 & -0.58 \\
\hline$A C F\left[\sigma_{x s}\left(E_{i}[d y]\right)\right]$ & $\begin{array}{c}0.88 \\
(0.06)\end{array}$ & 0.98 & $N / A$ & 0.98 & 0.85 & 0.98 \\
\hline$\sigma(d y)(\%)$ & $\begin{array}{c}3.34 \\
(0.42)\end{array}$ & 3.42 & 3.36 & 3.47 & 3.30 & 3.42 \\
\hline$\sigma(d c) / \sigma(d y)$ & $\begin{array}{c}0.65 \\
(0.04)\end{array}$ & 0.65 & 0.70 & 0.65 & 0.70 & 0.64 \\
\hline$\sigma(d i) / \sigma(d y)$ & $\begin{array}{c}4.45 \\
(0.46)\end{array}$ & 4.82 & 5.07 & 5.17 & 4.68 & 4.82 \\
\hline $\operatorname{cor}(d c, d i)$ & $\begin{array}{c}0.68 \\
(0.11)\end{array}$ & 0.42 & 0.24 & 0.28 & 0.38 & 0.42 \\
\hline $\operatorname{cor}\left(d c, r_{e x}^{l e v}\right)$ & $\begin{array}{c}0.15 \\
(0.17)\end{array}$ & 0.07 & -0.08 & 0.03 & -0.07 & 0.08 \\
\hline$E\left(r_{e x}^{l e v}\right)(\%)$ & $\begin{array}{l}3.89 \\
(2.35)\end{array}$ & 4.27 & 3.77 & 4.25 & 3.80 & 4.28 \\
\hline$\sigma\left(r_{e x}^{l e v}\right)$ & $\begin{array}{l}21.21 \\
(1.82)\end{array}$ & 13.36 & 13.21 & 16.18 & 9.55 & 13.37 \\
\hline$E[r](\%)$ & $\begin{array}{c}0.64 \\
(0.40)\end{array}$ & 0.99 & 1.24 & 1.01 & 1.23 & 0.99 \\
\hline$\sigma(r)(\%)$ & $\begin{array}{c}3.82 \\
(0.46)\end{array}$ & 1.28 & 1.90 & 1.28 & 1.91 & 1.27 \\
\hline$A C F\left[r_{e x}^{l e v}\right]$ & $\begin{array}{c}0.05 \\
(0.13)\end{array}$ & 0.00 & 0.00 & 0.00 & 0.00 & 0.00 \\
\hline$A C F[r]$ & $\begin{array}{c}0.7 \\
(0.06)\end{array}$ & 0.67 & 0.50 & 0.67 & 0.50 & 0.66 \\
\hline$A C F[d c]$ & $\begin{array}{c}0.51 \\
(0.17)\end{array}$ & 0.67 & 0.66 & 0.67 & 0.68 & 0.67 \\
\hline
\end{tabular}

Note: Target moments (column 1) and simulated moments from our benchmark model (column 2 ) and a model that allows for aggregate noise in the private signals instead of exogenous public signals (column 6). Columns 3-5 impose full rationality (column 3) and full rationality about only the long-run and short-run shocks to TFP growth, respectively, on the benchmark estimates. Lowercase letters denote logs. $E[],. \sigma($.$) , and \operatorname{cor}(.,$.$) denote means, standard deviations, and$ correlations, respectively. $d$ stands for the first difference in the time series (e.g., $\sigma(d y)$ stands for the standard deviation of output growth). $A C F[$.$] refers to the first-order autocorrelation. E_{\mathrm{i}}[$. denotes the one-period-ahead forecast of forecaster i, $\bar{E}[$.$] denotes the cross-sectional average$ of $E_{\mathrm{i}}[$.$] , and \sigma_{\mathrm{xs}}($.$) denotes the time-series average of the cross-sectional standard deviation of$ one-period-ahead forecasts. See section 3.2 of the main text for details on the construction of the target moments. 
dispersed private information and near-rational errors relative to noise contained in exogenous public signals. While both mechanisms can generate forecast errors of a given size by determining the amount of information available, only the former can simultaneously generate disagreement. Because the amount of disagreement we see in the data is large relative to the size of average forecast errors, our estimator prefers very noisy (and thus useless) public signals and instead chooses (intermediate) values of $\sigma_{\nu S}$ and $\sigma_{\nu L}$ that simultaneously imply large amplified nearrational errors and a maximum of disagreement in equilibrium. (We show below that this result continues to hold when we allow for aggregate noise in the private signal.)

Second, near-rational behavior increases the amount of risk faced by households because it delays the resolution of uncertainty: it raises the standard deviation of average forecast errors and lowers the standard deviation of average expectations relative to the fully rational case. Households react to this increase in risk by demanding a higher equity premium (4.27 vs. 3.77 in the fully rational case) and more precautionary savings, lowering the average risk-free rate by 0.25 percentage points relative to the fully rational case. Both of these effects are particularly pronounced because households have a preference for early resolution of uncertainty $(\psi>1)$.

Third, near-rational behavior (and the amplified errors it entails) distorts aggregate quantities. Comparing the remaining moments shown in columns 2 and 3 shows the effects of these distortions: a higher correlation between consumption growth and investment growth $(0.42$ vs. 0.24 in the fully rational case), a similar rise in the correlation between consumption growth and stock returns, a fall in the relative volatility of investment growth from (4.82 vs. 5.07 in the fully rational case), and a slight increase in the unconditional volatility of output and stock returns. All of these effects of near-rational behavior point to misallocations that result from changes in the aggregate dynamics of the economy.

Figure 5 illustrates the effects of these misallocations by comparing impulse-response functions of our benchmark model (the solid blue lines) with those under fully rational behavior (the red broken lines). The four columns depict responses to a two-standard-deviation shock to $\eta^{L}, \epsilon^{L}, \eta^{S}$, and $\epsilon^{S}$, respectively, where $\eta^{L}$ and $\eta^{S}$ materialize in period 1 and households form expectations (and make errors) in period 0 .

The plots in column 1 show that learning about the shock to long-run productivity in advance allows households to adjust their behavior ex ante (in period 0). Under full rationality, all of this adjustment occurs in period zero, allowing households to decrease consumption and increase investment in the period before the arrival of the long-run productivity shock. Under near-rational behavior, information is only partially revealed in period 0 such that households partially adjust their behavior in both periods 0 and 1 . This dampening of the response of investment and stock returns to long-run shocks is responsible for the increase in the correlation between consumption growth and investment as well as the increase in the correlation between consumption growth and stock returns noted above (compare columns 2 and 4 in Table 3, 
where the specification column 4 imposes $\sigma_{\epsilon L}=0$ ). More generally, this dampening also tends to decrease the unconditional volatility of other macroeconomic aggregates, such as output, investment, and stock returns - all signs of a delayed response to persistent shocks.

By contrast, columns 3 and 4 of Figure 5 show that near-rational behavior with respect to the short-run shock tends to increase the volatility of macroeconomic aggregates. Column 3 shows the impulse responses of consumption and investment with respect the the short-run productivity shock are similar, regardless of whether households are rational or near-rational. The reason is that, due to the transitory nature of the shock, households invest relatively little even when learning with certainty that short-run productivity will be higher next month. The amplitude of the response to the productivity shock is thus roughly the same regardless of near-rational behavior. However, when households are near-rational, the same aggregates also respond to the amplified near-rational error, increasing the unconditional volatility of investment, consumption, and stock returns (compare columns 2 and 5 in Table 3, where the specification column 5 imposes $\left.\sigma_{\epsilon S}=0\right) .^{18}$

We draw two main conclusions about the effect of near-rational behavior on aggregate quantities. First, near-rational behavior induces misallocations by delaying the response to information, in particular about $\eta^{L}$, and by inducing non-fundamental volatility. Second, these misallocations tend to have different effects on unconditional moments, contributing to the identification of the relative size of the two types of near-rational errors. For example, nearrational errors about $\eta^{L}$ have a larger effect on the correlation between consumption growth and investment because information about the long-run shock elicits a larger investment response ex-ante. Similarly, near-rational errors about $\eta^{L}$ tend to lower the unconditional variances of output, stock returns, and investment while near-rational errors about the $\eta^{S}$ tend to increase them. The differential effect on the standard deviation of excess returns is particularly large (16.18 when $\sigma_{\epsilon L}=0$ vs. 9.55 when $\sigma_{\epsilon S}=0$ ). Figure 6 shows comparative statics illustrating these differential effects for two examples.

We next study the impact of the three sets of effects of near-rational behavior on welfare. As in our static model, near-rational behavior is costly for society because it generates a dispersion of consumption and leisure across households, increases risk by delaying the resolution of uncertainty about the path of future consumption, and decreases aggregate wealth due to misallocations of consumption, labor, and investment. Although our quantitative model does not allow a simple additive separation of these three channels similar to the one we derived in section 1, we can gauge the relative influence of each of the three channels by calculating compensating variations associated with two counterfactual experiments.

In the first experiment, we gauge the welfare cost of the cross-sectional dispersion in con-

\footnotetext{
${ }^{18}$ The effect on the unconditional volatility of excess stock returns is particularly large and asymmetric because the increase in uncertainty about $\eta^{S}$ dampens off-setting movements in the risk-free rate.
} 

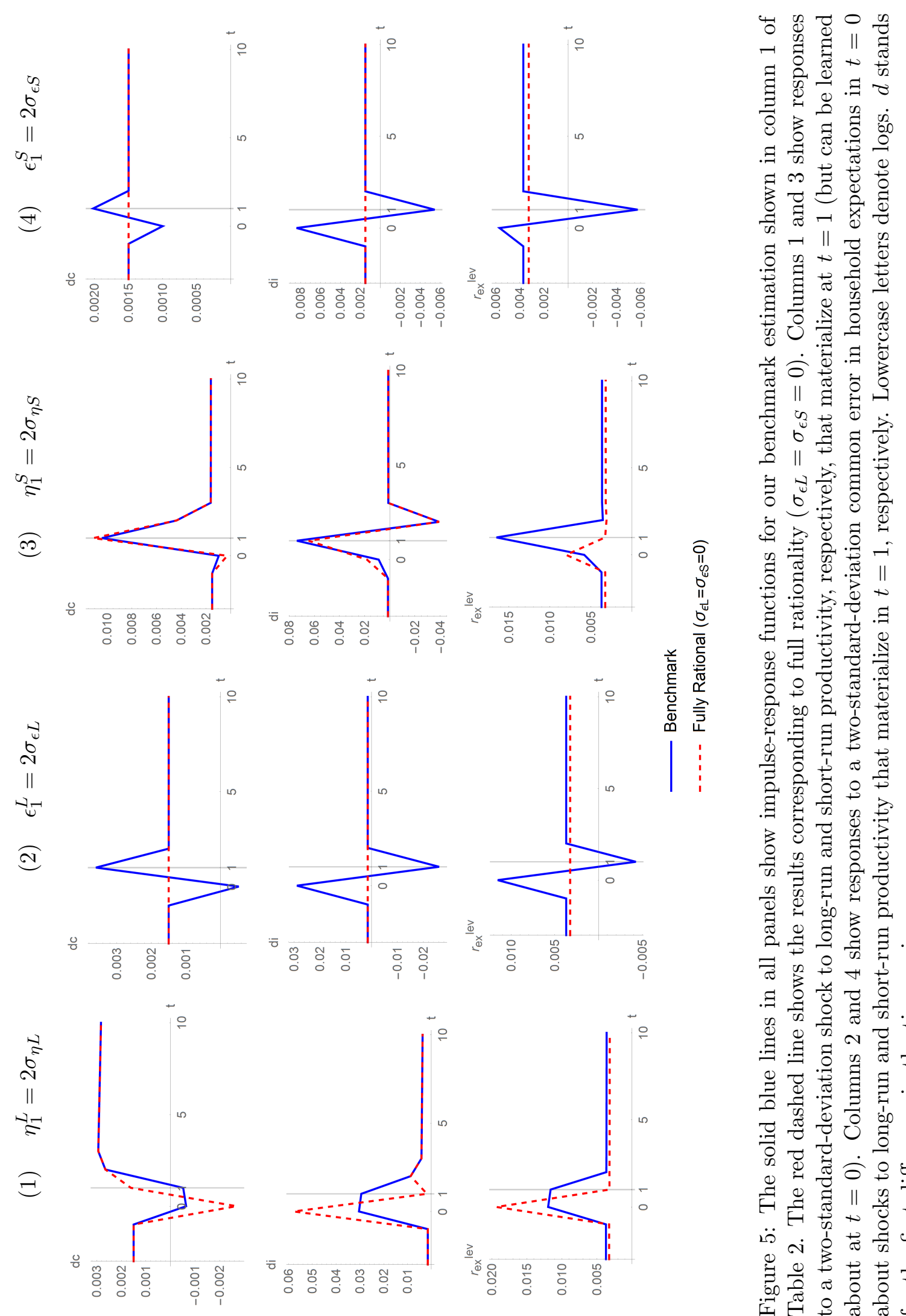

类 $\dot{0}$.

丞 $\|$ 票

-

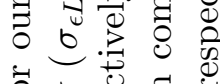

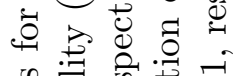

व :

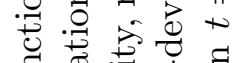

$\exists$ :

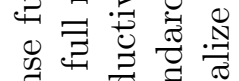

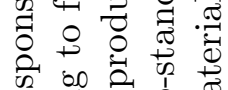

की

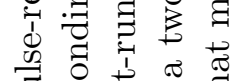

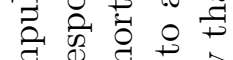

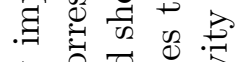

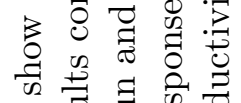

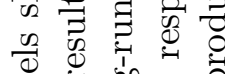

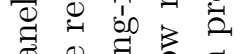

蛋

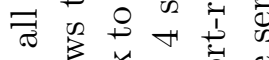

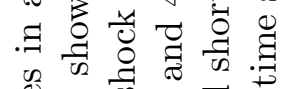

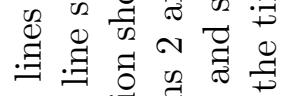

Ð

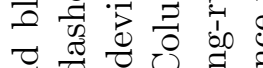

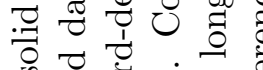

\&

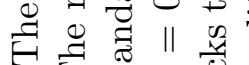

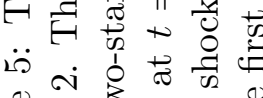

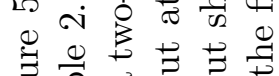

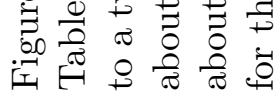


$\operatorname{cor}(\mathrm{dc}, \mathrm{di})$

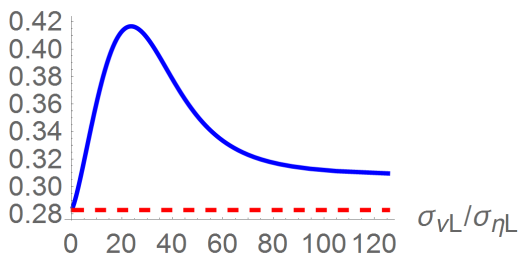

$\sigma(\mathrm{dy})(\%)$

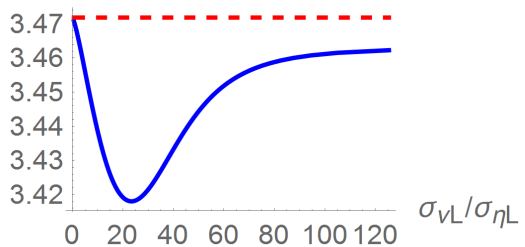

$\operatorname{cor}(\mathrm{dc}, \mathrm{di})$
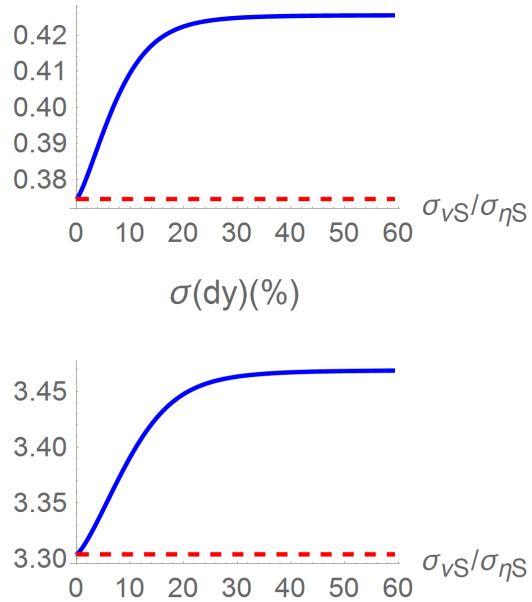

Figure 6: Comparative statics over dispersion of private information about the long-run (left column) and short-run (right column) shock to TFP growth $\left(\sigma_{\nu L}\right.$ and $\left.\sigma_{\nu S}\right)$. The red dashed line shows the results corresponding to full rationality $\left(\sigma_{\epsilon L}=\sigma_{\epsilon S}=0\right)$. See the caption of Figure 4 for details First row: correlation of consumption growth with investment growth; second row: standard deviation of output growth in percent;

sumption and leisure by calculating a household's willingness to pay for receiving the mean private signal in all periods $\left(\nu_{i t}=0 \forall t\right)$, while holding constant its policy functions and the coefficients in its expectations operator. The household is thus being offered to always consume at the mean of the distribution across households, holding constant all other aspects of its behavior.

In the second experiment, we gauge the direct utility cost of the delayed resolution of uncertainty by evaluating the stream of consumption and leisure accruing to households in our model using a CRRA utility function with $\gamma=\frac{1}{\psi}=10$ and then calculating the social cost of near-rational behavior in the usual way (i.e., by calculating the compensating variation for setting $\left.\mu_{j}=0 \forall j \neq i\right)$. Because the CRRA utility function is additively separable, it is invariant to the timing of resolution of uncertainty. Calculating the social cost of near-rational behavior in this way may thus serve as a useful reference for the size of the welfare effects of near-rational behavior absent its direct effect of delaying resolution of uncertainty.

Table 4 shows the results of the two counterfactual experiments. It shows that of the total social cost of near-rational behavior (5.31\%), a full 1.5 percentage points are accounted for by the cost of dispersion in consumption and leisure across households. ${ }^{19}$ Moreover, the social cost of near-rational behavior evaluated using the CRRA utility function is $1.06 \%$ of permanent consumption, suggesting that about four fifths of the social cost of near-rational behavior in our

\footnotetext{
${ }^{19}$ Recall that trading in contingent claims ensures all households hold the same wealth at the beginning of each period, such that this number reflects only the dispersion in behavior that arises within the period. In this sense, it is a lower bound for the cost of dispersion that would arise in a model with a dynamic wealth distribution.
} 
Table 4: Decomposition of Welfare Losses

\begin{tabular}{lcc}
\hline \hline & $\begin{array}{c}\text { Benchmark Model } \\
\text { (Noisy Public Signal) }\end{array}$ & $\begin{array}{c}\text { Aggregate Noise } \\
\text { in Private Signal }\end{array}$ \\
\hline Social Cost & & \\
Total $\left(\sigma_{\epsilon \mathrm{L}}, \sigma_{\epsilon \mathrm{S}}=0 \forall \mathrm{j} \neq \mathrm{i}\right)$ & 5.31 & 5.40 \\
from errors about $\operatorname{LRR}\left(\sigma_{\epsilon \mathrm{L}}=0 \forall \mathrm{j} \neq \mathrm{i}\right)$ & 5.02 & 5.11 \\
from errors about $\operatorname{SRR}\left(\sigma_{\epsilon \mathrm{S}}=0 \forall \mathrm{j} \neq \mathrm{i}\right)$ & 0.29 & 0.28 \\
Cost of dispersion & 1.50 & 1.50 \\
Social Cost evaluated using CRRA $(\gamma=10, \psi=1 / 10)$ & 1.06 & 1.08 \\
\hline \hline
\end{tabular}

Note: Implications of near-rational behavior for welfare based on the results of estimations shown in columns 1 and 2 of Table 2, respectively. Cost of dispersion: a household's willingness to pay for receiving the mean private signal in all periods $\left(\nu_{i t}=0 \forall t\right)$, holding constant its policy functions and the coefficients in its expectations operator. Social cost evaluated using CRRA: social cost of near-rational behavior when evaluating the stream of consumption and leisure accruing to households with $\gamma=\frac{1}{\psi}=10$. See the caption of Table 2 for details.

benchmark estimation arises due to its effect of delaying the resolution of uncertainty. The same preference for early resolution of uncertainty that allows the model to produce a relatively large equity premium (Bansal and Yaron, 2004) and large costs of aggregate consumption fluctuations (Epstein et al., 2014; Croce, 2012) thus also accounts for the majority of the social cost of nearrational behavior.

Consistent with these results, we also find that near-rational errors about long-run productivity are much more costly (5.02\% of permanent consumption) than those about short-run productivity $(0.29 \%)$, because they prevent households from learning about, and reacting preemptively to, the key determinant of the long-term path of their consumption.

In light of these findings, it is apparent that near-rational behavior and the non-fundamental volatility it induces is socially costly even when the capital stock is relatively unresponsive to variation in stock prices (and mispricings thus have a relatively smaller effect on the allocation of capital). Figure 7 shows comparative statics of the social cost of near-rational behavior over the elasticity of the capital stock with respect to stock prices, $\xi$, holding constant all other parameters of our benchmark model. The figure shows results for values of $\xi$ ranging from one half to double its value in our benchmark model (7). The right part of the figure shows the same comparative static for the standard deviation of investment for comparison. The plots show the social cost of a breakdown in the market's capacity to aggregate information is almost completely invariant to variation in the elasticity of the capital stock with respect to stock prices and to the size of the dynamic response of the capital stock to a given mispricing. In this sense, stock market dysfunctionality (induced by near-rational behavior) is socially costly even if it appears to be a "sideshow." 

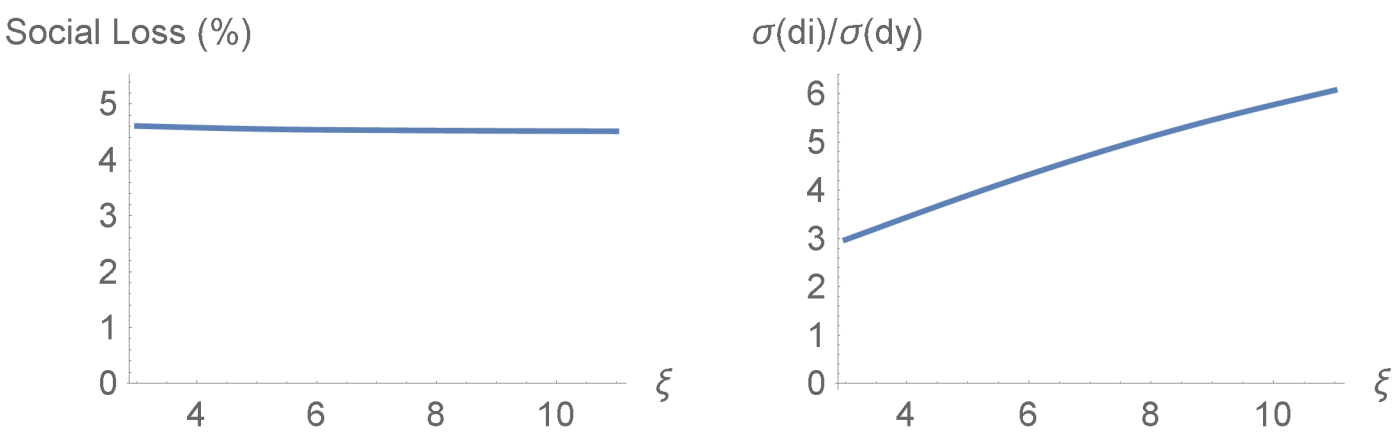

Figure 7: Comparative static of the social cost of near-rational behavior over the elasticity of the capital stock with respect to stock prices, $\xi$, holding constant all other parameters of our benchmark estimation shown in Table 2. The right part of the figure shows the same comparative static for the standard deviation of investment growth divided by the standard deviation of output growth for comparison.

\subsection{Robustness}

We next explore the robustness of our results across a range of different variations, considering alternative sources of noise in the information environment, variation in the degree of nearrationality, and the choice of the decision horizon.

Alternative Information Environments From the results in Table 2, we can see that the availability of an exogenous noisy public signal has only a minor effect on our estimates. Our benchmark estimates suggest these signals are relatively uninformative such that, in equilibrium, households largely ignore them when forming their expectations. As a result, the noise these exogenous signals transmit has little effect on equilibrium behavior.

Column 2 of Table 2 re-estimates a variation of our model where instead of exogenous noisy public signals, we allow for aggregate noise in private signals as in (32) and estimate the standard deviation of this aggregate noise along with $\sigma_{\nu j}, \sigma_{\epsilon j}(j=S, L)$, and $\sigma_{\eta S}$. We find again that our estimates do not load on this alternative source of noise $\left(\sigma_{\zeta L} / \sigma_{\eta L}=0\right.$ and $\left.\sigma_{\zeta S} / \sigma_{\eta S}=0.11\right)$. Looking across the two columns, all other estimates remain virtually unchanged, as do the implications of these estimates: the ratio of conditional to unconditional variance is 0.50 and 0.57 for the long-run and short-run shocks, respectively, near-rational errors are amplified by factors of 577 and 143, and the estimated social cost of near-rational behavior is now only slightly higher at $5.40 \%$ of permanent consumption. The last column in Table 3 shows that aggregate noise in the private signal also does not significantly affect the model's fit to the data.

Amplified near-rational errors thus appear more relevant for fitting the data than either of these alternative sources of noise operating through the signal structure. The reason is that, in 
the data, households make forecast errors and disagree (quite strongly) about their forecasts. Although noise from exogenous public signals and aggregate noise in private signals can rationalize only the former, near-rational behavior (in combination with noisy private signals) can rationalize forecast errors, disagreement, and the dynamics of this disagreement.

The Degree of Near-Rationality Because the literature has little precedent for assessing the minimum size of economic incentives required to induce households to engage in fully rational behavior, Table 5 probes the robustness of our results over various levels of $\bar{\lambda}$. The first four columns maintain the constraint $\lambda<\bar{\lambda}$ on the objective function (50) and vary this limit between $0.01 \%$ and $0.37 \%$ of consumption - the lowest and highest stakes at which natural experiments have found evidence inconsistent with rational consumption smoothing (see Appendix Table 1). Table 5 shows that our main results are largely unaffected across these variations. Even when we specify the private cost of near-rational behavior to be smaller than $0.01 \%$ of consumption, the then very small near-rational errors $\left(\sigma_{\epsilon L}=0.0008 \sigma_{\eta L}\right.$ and $\left.\sigma_{\epsilon S}=0.0001 \sigma_{\eta S}\right)$ are amplified enough to reduce the ratio of conditional to unconditional variance of the two shocks to about one half ( 0.49 and 0.62 , respectively), and the social cost of this near-rational behavior remains at $5.10 \%$ of permanent consumption. As we increase $\bar{\lambda}$, this social cost increases slightly up to $5.75 \%$ of permanent consumption (when $\bar{\lambda}=0.37 \%$ ). As the size of near-rational errors increases, the degree of equilibrium amplification decreases, as reflected in a decrease of the estimated idiosyncratic noise in the private signals $\sigma_{\nu L, S}$.

Column 2 alternatively sets $\bar{\lambda}=0.08 \%$, consistent with estimates of the private cost of rational inattention from Maćkowiak and Wiederholt (2015). ${ }^{20}$ The column again shows results remarkably close to those in our benchmark calibration.

Across all the variations in columns 1-4, our estimator always picks combinations of parameters at the maximum allowable private cost of near-rational behavior. Column 5 shows the results of an unconstrained estimation where we instead add the mean $(0.15 \%)$ and the variance (0.024) of the four numbers used as limits in the other columns to the target vector $\theta$ and the weighting matrix $\Omega$, respectively. In this case, the estimated private cost of near-rational behavior is even higher ( $0.71 \%$ of permanent consumption), but nevertheless about 8 -fold smaller than the social cost $(5.55 \%)$.

Our main conclusion from this table is that the less rational we assume households to be, the better the model tends to fit the data. Nevertheless, the central insight that the social cost of near-rational behavior is orders of magnitude higher than the private cost appears independent of how we specify this private cost.

\footnotetext{
${ }^{20}$ Maćkowiak and Wiederholt (2015) find that the per-period loss accruing to firms due to deviations of the price from the profit-maximizing price equals 1/1700 of firms' steady-state revenue and 1/5900 of steady-state consumption (pages 3 and 31 ), collectively amounting to approximately $0.08 \%$ of consumption.
} 
Table 5: Estimation using alternative targets for private cost of near-rational behavior

\begin{tabular}{|c|c|c|c|c|c|}
\hline & $(1)$ & $(2)$ & $(3)$ & $(4)$ & $(5)$ \\
\hline & $\lambda \leq 0.01$ & $\lambda \leq 0.08$ & $\begin{array}{c}\lambda \leq 0.13 \\
\text { (Benchmark) }\end{array}$ & $\lambda \leq 0.37$ & unconstrained \\
\hline \multicolumn{6}{|l|}{ Implications for Welfare } \\
\hline Private Cost & 0.01 & 0.08 & 0.13 & 0.37 & 0.71 \\
\hline Total Social Cost & 5.1 & 5.12 & 5.31 & 5.75 & 5.55 \\
\hline \multicolumn{6}{|c|}{ Implications for Information Aggregation } \\
\hline$V_{t}[\eta L] / \sigma_{\eta L}^{2}$ & 0.49 & 0.49 & 0.49 & 0.5 & 0.41 \\
\hline$V_{t}[\eta S] / \sigma_{\eta S}^{2}$ & 0.62 & 0.58 & 0.56 & 0.5 & 0.48 \\
\hline \multicolumn{6}{|l|}{ Estimated Parameters } \\
\hline$\sigma_{\epsilon \mathrm{L}} / \sigma_{\eta \mathrm{L}}(\%)$ & 0.08 & 0.08 & 0.09 & 0.31 & 0.18 \\
\hline$\sigma_{\epsilon \mathrm{S}} / \sigma_{\eta \mathrm{S}}(\%)$ & 0.01 & 0.34 & 0.42 & 0.92 & 2.31 \\
\hline$\sigma_{\nu \mathrm{L}} / \sigma_{\eta \mathrm{L}}$ & 24.35 & 25.82 & 23.6 & 12.82 & 21.42 \\
\hline$\sigma_{\nu \mathrm{S}} / \sigma_{\eta \mathrm{S}}$ & 158.87 & 17.74 & 13.61 & 7.39 & 5.35 \\
\hline$\sigma_{\eta \mathrm{S}}(\%)$ & 0.98 & 0.98 & 0.98 & 0.98 & 0.98 \\
\hline$\sigma_{\varpi \mathrm{L}} / \sigma_{\eta \mathrm{L}}$ & 3.00 & 3.00 & 3.69 & $10^{13}$ & 0.90 \\
\hline$\sigma_{\varpi \mathrm{S}} / \sigma_{\eta \mathrm{S}}$ & 1.53 & 1.53 & 1.99 & $10^{12}$ & 1.38 \\
\hline
\end{tabular}

Note: Variations of the benchmark estimation from Table 2 using different constraints on the private cost of near-rational behavior $(\lambda)$. Column 3 shows the benchmark estimation for comparison. Column 5 shows the results of an unconstrained estimation where we instead add the mean and the variance of the four numbers shown $(0.01,0.08,0.13$, and 0.37$)$ to the target vector and the weighting matrix of our estimator. See section 3.4 of the main text for details.

Choice of Decision Horizon As a final robustness check, we explore how our results change when we use a quarterly rather than a monthly decision horizon, where households then receive signals about long-run and short-run shocks to productivity one quarter ahead of time. To tie our hands, we again use the quarterly calibration proposed by Croce (2014) (he simply scales the time discount factor and the depreciation rate such that $\delta^{4}=0.954$ and $4 \delta_{k}=0.06$, but sets $\rho^{4}=0.9$ ), and then use the same parameters from column 1 of Table 2 while scaling up the standard deviations of all shocks by $\sqrt{3}$. This calibration gives a similarly good fit to the data and shows a social cost of near-rational behavior of $6.34 \%$ of permanent consumption. Our results thus appear to imply only moderately larger social costs when we increase the horizon at which households can learn about the future.

\section{Conclusion}

A large literature on noisy rational expectations studies markets' capacity to aggregate dispersed information. However, normative and quantitative applications of this literature have often faced difficulties because they require a source of noise in demand, a role often filled by noise traders 
whose welfare is hard to evaluate, or by some other large shock to the supply of financial assets that may be hard to rationalize in general equilibrium.

In this paper, we have proposed a microfoundation for noisy demand that is part and parcel of the idea that prices aggregate information: an individual household does not internalize that small errors it makes when forming expectations affect other households' ability to learn about the future. If these errors are, in any part, correlated across households, they amplify as households learn from equilibrium prices, leading to much larger errors in equilibrium expectations and diminishing the market's capacity to aggregate information. We argue that this externality provides a logically coherent rationale for why equilibrium asset prices and other economic aggregates might contain large amounts of non-fundamental noise even if all economic actors appear to act rationally and financial markets are efficient in the sense that rational investors cannot systematically outperform the market.

We find that the more dispersed private information is across households, the more severe this externality is - making small mistakes is cheaper precisely when they are most socially harmful. The social costs of this near-rational behavior result from misallocations of resources, increases in the variance of consumption across households, and delays in the resolution of uncertainty.

A quantitative application of our model yields four main insights. First, most of the information about future productivity that households receive originates from noisy private rather than public signals. Markets thus play a potentially important role in aggregating this information. The social value of aggregating all of it amounts to $9.84 \%$ of permanent consumption. Second, amplified near-rational errors drive out about half of this information content, such that equilibrium asset prices transmit as much amplified noise as information. Third, the reduced information content of asset prices results in a countercyclical and highly persistent dispersion of expectations across households, in line with salient stylized facts. It also results in a rise of the equity premium, a fall in the risk-free interest rate, and a range of distortions in aggregate investment, output, and consumption. Fourth, our estimates for the social cost of near-rational behavior range between $5.1 \%$ and $5.7 \%$ of permanent consumption, whereas the private cost of this behavior is on the order of $0.13 \%$ of permanent consumption.

We believe that the framework developed in this paper may prove useful for a normative and quantitative evaluation of a large body of results from the market microstructure literature (Hellwig, 1980; Kyle, 1985), studying the role of dispersed information in the economy, its effect on macroeconomic dynamics, the cross-section of household behavior, and its interactions with policy. Particularly interesting would be a re-evaluation of the social value of the provision of private information (Grossman and Stiglitz, 1980). Beyond the market microstructure literature, near-rational behavior may also provide a new approach to other contexts where agents' ability to infer information from prices has real effects, for example on the slope of the Philips curve (Lucas, 1975). 


\section{References}

Aaronson, D., S. Agarwal, and E. French (2012). The spending and debt response to minimum wage hikes. American Economic Review 102(7), 3111-39.

Agarwal, S., C. Liu, and N. S. Souleles (2007). The reaction of consumer spending and debt to tax rebates-evidence from consumer credit data. Journal of Political Economy 115(6), 986-1019.

Agarwal, S. and W. Qian (2014). Consumption and debt response to unanticipated income shocks: Evidence from a natural experiment in singapore. American Economic Review 104(12), 4205-30.

Akerlof, G. and J. Yellen (1985). A near-rational model of the business cycle with wage and price inertia. Quarterly Journal of Economics, 823-838.

Albagli, E. (2011). Amplification of uncertainty in illiquid markets. Available at SSRN 1788216.

Albuquerque, R., M. S. Eichenbaum, and S. Rebelo (2015). Valuation risk and asset pricing. mimeo Northwestern University.

Allen, F. and D. Gale (2003). Asset price bubbles and monetary policy. Desai, Meghnad, and Yahia Said, eds. Global Governance and Financial Crises, Routledge, London and New York, 19.

Allen, F., S. Morris, and H. S. Shin (2006). Beauty contests and iterated expectations in asset markets. Review of Financial Studies 13 (3), 719-752.

Amador, M. and P.-O. Weill (2012). Learning from private and public observations of others? actions. Journal of Economic Theory 147(3), 910-940.

Angeletos, G.-M. and J. La'O (2013). Sentiments. Econometrica 81(2), 739-779.

Angeletos, G.-M., G. Lorenzoni, and A. Pavan (2012). Wall street and silicon valley: A delicate interaction. NBER Working Paper No. 13475.

Bacchetta, P. and E. Van Wincoop (2008). Higher order expectations in asset pricing. Journal of Money, Credit and Banking 40(5), 837-866.

Baker, M., J. C. Stein, and J. Wurgler (2003). When does the market matter? stock prices and the investment of equity-dependent firms. Quarterly Journal of Economics, 969-1005.

Bansal, R., D. Kiku, and A. Yaron (2012). An empirical evaluation of the long-run risks model for asset prices. Critical Finance Review 1(1), 183-221. 
Bansal, R. and A. Yaron (2004). Risks for the long run: A potential resolution of asset pricing puzzles. Journal of Finance, 1481-1509.

Barberis, N., A. Shleifer, and R. Vishny (1998). A model of investor sentiment. Journal of Financial Economics 49, 307-343.

Barlevy, G. (2005). The cost of business cycles and the benefits of stabilization. Economic Perspectives-Federal Reserve Bank of Chicago 29 (1), 32.

Bikhchandani, S., D. Hirshleifer, and I. Welch (1998). Learning from the behavior of others: Conformity, fads, and informational cascades. Journal of Economic Perspectives 12, 151-170.

Blanchard, O. J., C. Rhee, and L. H. Summers (1993). The stock market and investment. Quarterly Journal of Economics 108, 115-136.

Bloom, N. (2014). Fluctuations in uncertainty. Journal of Economic Perspectives 28(2), 153-76.

Broda, C. and J. A. Parker (2014). The economic stimulus payments of 2008 and the aggregate demand for consumption. Journal of Monetary Economics 68, Supplement(0), 20-36.

Browning, M. and M. D. Collado (2001). The response of expenditures to anticipated income changes: Panel data estimates. American Economic Review 91 (3), 681-692.

Brunnermeier, M., A. Simsek, and W. Xiong (2014). A welfare criterion for models with distorted beliefs. Quaterly Journal of Economics 129(4), 1711-1752.

Burnside, C., M. Eichenbaum, and S. Rebelo (2011). Understanding booms and busts in housing markets. National Bureau of Economic Research No. w16734.

Chauvin, K., D. Laibson, and J. Mollerstrom (2011). Asset bubbles and the cost of economic fluctuations. Journal of Money, Credit and Banking 43(s1), 233-260.

Chetty, R. (2012). Bounds on elasticities with optimization frictions: A synthesis of micro and macro evidence on labor supply. Econometrica 80(3), 969-1018.

Chirinko, R. S. and H. Schaller (2006). Fundamentals, misvaluation and investment: The real story. Institute for Advanced Studies, Economic Series 200.

Cochrane, J. H. (1989). The sensitivity of tests of the intertemporal allocation of consumption to near-rational alternatives. American Economic Review 79(3), 319-337.

Coibion, O. and Y. Gorodnichenko (2012). What can survey forecasts tell us about information rigidities? Journal of Political Economy 120(1), 116-159. 
Coulibaly, B. and G. Li (2006). Do homeowners increase consumption after the last mortgage payment? an alternative test of the permanent income hypothesis. Review of Economics and Statistics 88(1), 10-19.

Croce, M. (2012). Welfare costs in the long run. UNC Kenan-Flagler Research Paper (2013-3).

Croce, M. M. (2014). Long-run productivity risk: A new hope for production-based asset pricing? Journal of Monetary Economics 66(C), 13-31.

Daniel, K. D., D. Hirshleifer, and A. Subrahmanyam (2001). Overconfidence, arbitrage, and equilibrium asset pricing. Journal of Finance 56, 921-965.

DeLong, B. J., A. Shleifer, L. H. Summers, and R. J. Waldmann (1989). The size and incidence of the losses from noise trading. Journal of Finance 44, 681-696.

Diamond, D. W. and R. E. Verrecchia (1981). Information aggregation in a noisy rational expectations economy. Journal of Financial Economics 9(3), 221-235.

Dupor, B. (2005). Stabilizing non-fundamental asset price movements under discretion and limited information. Journal of Monetary Economics 52, 727-747.

Epstein, L., E. Farhi, and T. Stralezcki (2014). How much would you pay to resolve long-run risk? American Economic Review 104 (9), 2680-2697.

Epstein, L. G. and S. E. Zin (1989). Substitution, risk aversion, and the temporal behavior of consumption and asset returns: A theoretical framework. Econometrica 57 (4), 937-969.

Farhi, E. and S. Panageas (2006). The real effects of stock market mispricing at the aggregate: Theory and empirical evidence. mimeo Harvard University.

Fuchs-Schuendeln, N. and T. A. Hassan (2015, June). Natural experiments in macroeconomics. (21228).

Galeotti, M. and F. Schiantarelli (1994). Stock market volatility and investment: Do only fundamentals matter? Economica (New Series) 61, 147-165.

Gilchrist, S., C. P. Himmelberg, and G. Huberman (2005). Do stock price bubbles influence corporate investment? Journal of Monetary Economics 52, 805-827.

Goldstein, I., E. Ozdenoren, and K. Yuan (2013). Trading frenzies and their impact on real investment. Journal of Financial Economics.

Grossman, S. (1976). On the efficiency of competitive stock markets where trades have diverse information. Journal of Finance 31 (2), 573-585. 
Grossman, S. J. and J. E. Stiglitz (1980). On the impossibility of informationally efficient markets. American Economic Review 70 (3), 393-408.

Hassan, T. A. and T. M. Mertens (2011). Market sentiment: A tragedy of the commons. American Economic Review 101, 2.

Hellwig, C. (2005). Heterogenous information and the welfare effects of public information disclosures. mimeo Univeristy of California Los Angeles.

Hellwig, M. (1980). On the aggregation of information in competitive markets. Journal of Economic Theory 22, 477-498.

Hong, H. and J. C. Stein (1999). A unified theory of underreaction, momentum trading, and overreaction in asset markets. Journal of Finance 54, 2143-2184.

Hsieh, C.-T. (2003). Do consumers react to anticipated income changes? evidence from the alaska permanent fund. American Economic Review 93(1), 397-405.

Jermann, U. J. (1998). Asset pricing in production economies. Journal of Monetary Economics $41(2), 257-275$.

Johnson, D. S., J. A. Parker, and N. S. Souleles (2006). Household Expenditure and the Income Tax Rebates of 2001. American Economic Review 96 (5), 1589-1610.

Judd, K. L. (1998). Numerical Methods in Economics. The MIT Press.

Judd, K. L. (2002). Perturbation methods with nonlinear change of variables. mimeo, Hoover Institution, Stanford University.

Judd, K. L. and S.-M. Guu (2001). Asymptotic methods for asset market equilibrium analysis. Economic Theory 18(1), 127-157.

Kyle, A. (1985). Continuous auctions and insider trading. Econometrica.

Lansing, K. J. (2012). Speculative growth, overreaction, and the welfare cost of technologydriven bubbles. Journal of Economic Behavior \&3 Organization 83(3), 461-483.

Lorenzoni, G. (2009). A theory of demand shocks. American Economic Review 99 (5), 20502084.

Lucas, R. (1975). An equilibrium model of the business cycle. Journal of Political Economy 83(6), 1113-1144.

Maćkowiak, B. and M. Wiederholt (2015). Business cycle dynamics under rational inattention*. The Review of Economic Studies, rdv027. 
Mankiw, N. G. (1985). Small menu costs and large business cycles: A macroeconomic model of monopoly. Quarterly Journal of Economics 100 (2), 529-537.

Mertens, T. (2009). Excessively volatile stock markets: Equilibrium computation and policy analysis. mimeo New York University.

Mertens, T. M. and K. L. Judd (2013). Equilibrium existence and approximation for incomplete market models with substantial heterogeneity. mimeo New York University.

Morck, R., A. Shleifer, and R. W. Vishny (1990). The stock market and investment: Is the market a sideshow? Brookings Papers on Economic Activity 2, 157-215.

Odean, T. (1998). Volume, volatility, price, and profit: When all traders are above average. Journal of Finance 53, 1887-1934.

Odean, T. (1999). Do investors trade too much? American Economic Review 89, 1279-1298.

Panageas, S. (2005). Neoclassical theory of investment in speculative markets. mimeo the Wharton School.

Parker, J. A. (1999). The Reaction of Household Consumption to Predictable Changes in Social Security Taxes. American Economic Review 89(4), pp. 959-973.

Parker, J. A., N. S. Souleles, D. S. Johnson, and R. McClelland (2013). Consumer spending and the economic stimulus payments of 2008. American Economic Review 103(6), 2530-2553.

Patton, A. J. and A. Timmermann (2010). Why do forecasters disagree? lessons from the term structure of cross-sectional dispersion. Journal of Monetary Economics 57(7), 803-820.

Polk, C. and P. Sapienza (2009). The stock market and corporate investment: a test of catering theory. Review of Financial Studies 22, 187-217.

Qiu, W. and J. Wang (2010). Asset pricing under heterogeneous information. mimeo MIT Sloan School of Management.

Rauh, J. D. and A. Sufi (2012). Explaining corporate capital structure: Product markets, leases, and asset similarity. Review of Finance 16(1), 115-155.

Scholnick, B. (2013). Consumption Smoothing after the Final Mortgage Payment: Testing the Magnitude Hypothesis. Review of Economics and Statistics 95 (4), 1444-1449.

Shea, J. (1995). Union Contracts and the Life-Cycle/Permanent-Income Hypothesis. American Economic Review 85(1), pp. 186-200.

Sockin, M. (2015). Not so great expectations: A model of growth and informational frictions. mimeo Univerisity of Texas at Austin. 
Souleles, N. S. (1999). The Response of Household Consumption to Income Tax Refunds. American Economic Review 89 (4), 947-958.

Souleles, N. S. (2000). College tuition and household savings and consumption. Journal of Public Economics 77(2), 185 - 207.

Souleles, N. S. (2002, July). Consumer response to the Reagan tax cuts. Journal of Public Economics 85(1), 99-120.

Stein, J. C. (1987). Informational externalities and welfare-reducing speculation. Journal of Political Economy 95 (6), 1123-1145.

Stephens, M. (2008). The Consumption Response to Predictable Changes in Discretionary Income: Evidence from the Repayment of Vehicle Loans. Review of Economics and Statistics $90(2), 241-252$.

Wang, J. (1994). A model of competitive stock trading volume. Journal of Political Economy 102 (1), 127-168.

Woodford, M. (2010). Robustly optimal monetary policy with near-rational expectations. American Economic Review 100(1), 274-303.

Yu, J. (2011). Disagreement and return predictability of stock portfolios. Journal of Financial Economics 99, 162-183. 


\section{Online Appendix}

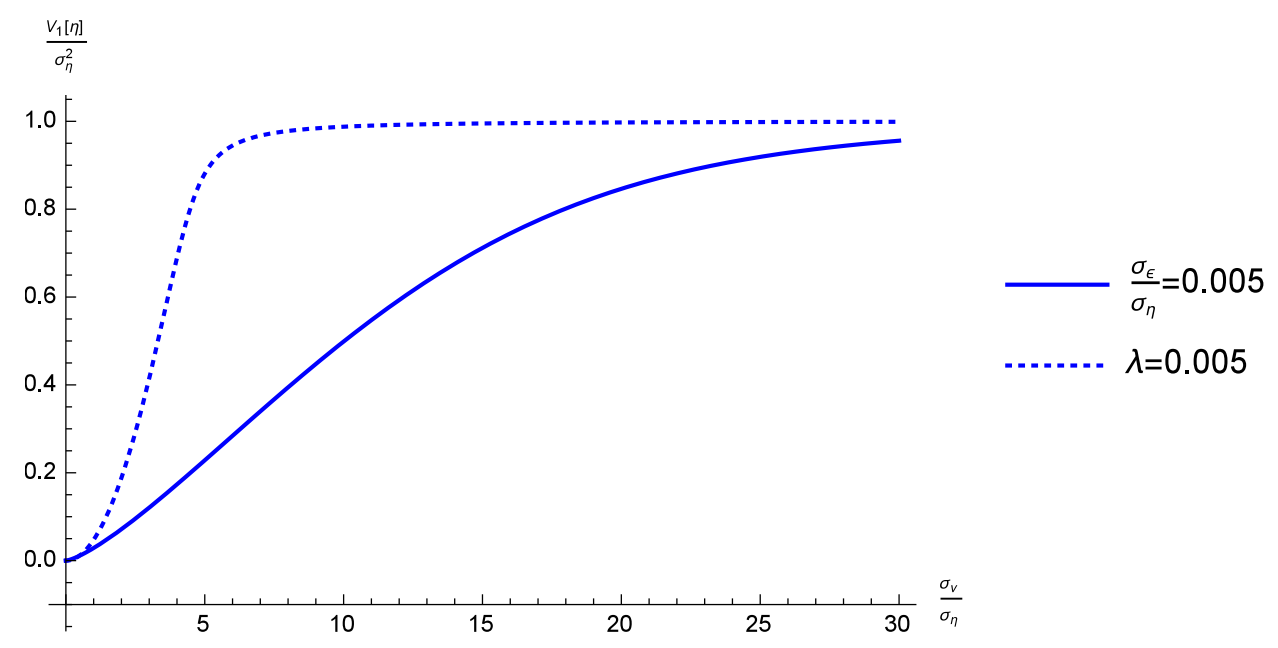

Appendix Figure 1: Ratio of the conditional variance of $\eta$ to its unconditional variance plotted over the level of dispersion of private information. The graph compares the case of an endogenous $\sigma_{\epsilon}$ with fixed $\lambda$ to case of a fixed $\sigma_{\epsilon}$ using $\kappa=2$ and $\rho=5$.

\section{A Appendix to Section 1}

\section{A.1 Derivation of (14), (15), and (27)}

Plugging (13) back into (10) and matching coefficients with (11) yields

$$
\pi_{0}=\frac{\alpha_{0}+\rho V_{1}[\eta] \kappa\left(1-\alpha_{2}\right)}{\left(1-\alpha_{2}\right)\left(1+\rho V_{1}[\eta] \kappa\right)}, \quad \pi_{1}=\frac{\alpha_{1}}{\left(1-\alpha_{2}\right)\left(1+\rho V_{1}[\eta] \kappa\right)}, \quad \gamma=\frac{1}{\left(1-\alpha_{2}\right)\left(1+\rho V_{1}[\eta] \kappa\right)}
$$

Using (2) and (11), the vector $\left(\eta, s_{i}, Q\right)$ has unconditional expectation $\left(\bar{\eta}, \bar{\eta}, \pi_{0}+\pi_{1} \bar{\eta}\right)$ and the following variance covariance matrix:

$$
\Sigma=\left(\begin{array}{ccc}
\sigma_{\eta}^{2} & \sigma_{\eta}^{2} & \pi_{1} \sigma_{\eta}^{2} \\
\sigma_{\eta}^{2} & \sigma_{\eta}^{2}+\sigma_{\nu}^{2} & \pi_{1} \sigma_{\eta}^{2} \\
\pi_{1} \sigma_{\eta}^{2} & \pi_{1} \sigma_{\eta}^{2} & \pi_{1}^{2} \sigma_{\eta}^{2}+\gamma^{2} \sigma_{\epsilon}^{2}
\end{array}\right)
$$


Thus, by the property of the conditional variance of the multi-normal distribution,

$$
\begin{aligned}
V_{1}[\eta] & =\sigma_{\eta}^{2}-\left(\begin{array}{cc}
\sigma_{\eta}^{2} & \pi_{1} \sigma_{\eta}^{2}
\end{array}\right)\left(\begin{array}{cc}
\sigma_{\eta}^{2}+\sigma_{\nu}^{2} & \pi_{1} \sigma_{\eta}^{2} \\
\pi_{1} \sigma_{\eta}^{2} & \pi_{1}^{2} \sigma_{\eta}^{2}+\gamma^{2} \sigma_{\epsilon}^{2}
\end{array}\right)^{-1}\left(\begin{array}{c}
\sigma_{\eta}^{2} \\
\pi_{1} \sigma_{\eta}^{2}
\end{array}\right) \\
& =\frac{1}{\sigma_{\eta}^{-2}+\left(\pi_{1}^{2} \gamma^{-2} \sigma_{\epsilon}^{-2}+\sigma_{\nu}^{-2}\right)}
\end{aligned}
$$

Plugging (51) and (52) into this expression gives (14).

Similarly, by the properties of the multi-normal distribution,

$$
E\left[\eta \mid s_{i}, Q\right]=\bar{\eta}+\left(\begin{array}{cc}
\sigma_{\eta}^{2} & \pi_{1} \sigma_{\eta}^{2}
\end{array}\right)\left(\begin{array}{cc}
\sigma_{\eta}^{2}+\sigma_{\nu}^{2} & \pi_{1} \sigma_{\eta}^{2} \\
\pi_{1} \sigma_{\eta}^{2} & \pi_{1}^{2} \sigma_{\eta}^{2}+\gamma^{2} \sigma_{\epsilon}^{2}
\end{array}\right)^{-1}\left(\begin{array}{c}
s_{i}-\bar{\eta} \\
Q-\left(\pi_{0}+\pi_{1} \bar{\eta}\right)
\end{array}\right) .
$$

Replacing $Q$ by (10) and plugging in (13) and (53) gives (15).

Matching the coefficients of (15) with (12)

$$
\left(\begin{array}{c}
\alpha_{1} \\
\alpha_{2}\left(1+\rho V_{1}[\eta] \kappa\right)
\end{array}\right)=\left(\begin{array}{cc}
\sigma_{\eta}^{2} & \pi_{1} \sigma_{\eta}^{2}
\end{array}\right)\left(\begin{array}{cc}
\sigma_{\eta}^{2}+\sigma_{\nu}^{2} & \pi_{1} \sigma_{\eta}^{2} \\
\pi_{1} \sigma_{\eta}^{2} & \pi_{1}^{2} \sigma_{\eta}^{2}+\gamma^{2} \sigma_{\epsilon}^{2}
\end{array}\right)^{-1}
$$

and solving for $\alpha_{1}, \alpha_{2}$ yields

$$
\begin{aligned}
\alpha_{1} & =\frac{\gamma^{2} \sigma_{\eta}^{2} \sigma_{\epsilon}^{2}}{\gamma^{2} \sigma_{\nu}^{2} \sigma_{\epsilon}^{2}+\sigma_{\eta}^{2}\left(\pi_{1}^{2} \sigma_{\nu}^{2}+\gamma^{2} \sigma_{\epsilon}^{2}\right)}, \\
\alpha_{2} & =\frac{\pi_{1} \sigma_{\eta}^{2} \sigma_{\nu}^{2}}{\left(\gamma^{2} \sigma_{\nu}^{2} \sigma_{\epsilon}^{2}+\sigma_{\eta}^{2}\left(\pi_{1}^{2} \sigma_{\nu}^{2}+\gamma^{2} \sigma_{\epsilon}^{2}\right)\right)\left(1+\rho V_{1}[\eta] \kappa\right)} .
\end{aligned}
$$

Combining (54) with (51), (52), and (14) yields (27).

\section{A.2 Proof of Lemma 1.1}

Use the law of total variance and (11) and (12) to get

$$
\begin{aligned}
\sigma_{\eta}^{2} & =V_{1}[\eta]+V_{0}\left[E_{1 i}[\eta]\right] \\
& =V_{1}[\eta]+V_{0}\left[\alpha_{1} \nu_{i}+\left(\alpha_{1}+\alpha_{2} \pi_{1}\left(1+\rho V_{1}[\eta] \kappa\right)\right) \eta+\alpha_{2} \gamma \epsilon\left(1+\rho V_{1}[\eta] \kappa\right)\right] \\
& =V_{1}[\eta]+\alpha_{1}^{2} \sigma_{\nu}^{2}+\left(\alpha_{1}+\alpha_{2} \pi_{1}\left(1+\rho V_{1}[\eta] \kappa\right)\right)^{2} \sigma_{\eta}^{2}+\alpha_{2}^{2} \gamma^{2} \sigma_{\epsilon}^{2}\left(1+\rho V_{1}[\eta] \kappa\right)^{2} .
\end{aligned}
$$

Now note from (17) and (18) that

$$
\alpha_{1}^{2} \sigma_{\nu}^{2}+\alpha_{2}^{2} \gamma^{2} \sigma_{\epsilon}^{2}\left(1+\rho V_{1}[\eta] \kappa\right)^{2}=\frac{V_{1}[\eta]^{2}}{\sigma_{\nu}^{2}}+\frac{V_{1}[\eta]^{4}}{\sigma_{\nu}^{4} \sigma_{\epsilon}^{2}}=\frac{V_{1}[\eta]^{2}}{\sigma_{\nu}^{2}}+\frac{V_{1}[\eta]^{2} \alpha_{1}^{2}}{\sigma_{\epsilon}^{2}},
$$


and from (14) that $\frac{\alpha_{1}^{2}}{\sigma_{\epsilon}^{2}}=\frac{1}{V_{1}[\eta]}-\left(\sigma_{\eta}^{-2}+\sigma_{\nu}^{-2}\right)$ such that

$$
\alpha_{1}^{2} \sigma_{\nu}^{2}+\alpha_{2}^{2} \gamma^{2} \sigma_{\epsilon}^{2}\left(1+\rho V_{1}[\eta] \kappa\right)^{2}=V_{1}[\eta]-V_{1}[\eta]^{2} \sigma_{\eta}^{-2} .
$$

In addition, using (51) we can show that

$$
\begin{aligned}
\left(\alpha_{1}+\alpha_{2} \pi_{1}\left(1+\rho V_{1}[\eta] \kappa\right)\right)^{2} & =\left(\left(1-\alpha_{2}\right) \pi_{1}\left(1+\rho V_{1}[\eta] \kappa\right)+\alpha_{2} \pi_{1}\left(1+\rho V_{1}[\eta] \kappa\right)\right)^{2} \\
& =\pi_{1}^{2}\left(1+\rho V_{1}[\eta] \kappa\right)^{2}
\end{aligned}
$$

Substituting these two expressions back into (55) yields

$$
\sigma_{\eta}^{2}=2 V_{1}[\eta]-V_{1}[\eta]^{2} \sigma_{\eta}^{-2}+\pi_{1}^{2}\left(1+\rho V_{1}[\eta] \kappa\right)^{2} \sigma_{\eta}^{2}
$$

Solving this expression for $V_{1}[\eta]$ gives

$$
V_{1}[\eta]=\frac{\sigma_{\eta}^{2}\left(1-\pi_{1}\right)}{1+\kappa \rho \pi_{1} \sigma_{\eta}^{2}} .
$$

Now take the market-clearing condition (8), plug in (7) and (9) on the left-hand side and (1) on the right to get

$$
\frac{\int_{0}^{1} E_{1 i}[\eta] d i-Q+\epsilon}{\rho V_{1}[\eta]}=\kappa(Q-1) .
$$

Take the unconditional expectation on both sides:

$$
E_{0}[\eta-Q]=\rho \kappa V_{1}[\eta]\left(E_{0}[Q]-1\right)
$$

Now note from (11) that $E_{0}[Q]=\pi_{0}+\pi_{1} \bar{\eta}$ and therefore:

$$
-\pi_{0}+\left(1-\pi_{1}\right) \bar{\eta}=\rho \kappa V_{1}[\eta]\left(E_{0}[Q]-1\right) .
$$

Solving for $\pi_{0}$ and plugging in (56) yields

$$
\pi_{0}=\frac{\left(1-\pi_{1}\right)\left(\bar{\eta}+\kappa \rho \sigma_{\eta}^{2}\right)}{1+\kappa \rho \sigma_{\eta}^{2}} .
$$

Similarly, from (51), (52), and (17), it follows that

$$
\gamma=\pi_{1} \frac{\sigma_{\nu}^{2}}{V_{1}[\eta]} .
$$


Again plugging in (56) yields

$$
\gamma=\frac{\pi_{1} \sigma_{\nu}^{2}\left(1+\kappa \rho \pi_{1} \sigma_{\eta}^{2}\right)}{\sigma_{\eta}^{2}\left(1-\pi_{1}\right)}
$$

To solve for $\pi_{1}$, substitute (17), (18), and (58) into (51) to get

$$
\pi_{1}=\sigma_{\nu}^{-2}\left(V_{1}[\eta]^{-1}-\frac{V_{1}[\eta]^{2}}{\pi_{1} \sigma_{\nu}^{4} \sigma_{\epsilon}^{2}}+\rho \kappa\right)^{-1} .
$$

Combining this expression with (56) and solving yields (20). Plugging (20) into (57) and (58) separately gives (19) and (21). And substituting $\alpha_{1}$ using (17) in (14) yields (22).

\section{A.3 Details on Amplification from Cost Function}

\section{Lemma A.1}

There is a one-to-one mapping between the cost parameter $\lambda$ and the size of the error $\sigma_{\epsilon}$ where the derivative $\frac{d \sigma_{\epsilon}}{d \lambda}$ is strictly positive. In the limit in which $\lambda \rightarrow 0$, all households behave fully rationally with $\sigma_{\epsilon} \rightarrow 0$.

To show the magnitude of near-rational errors, take the first-order condition with respect to the optimal choice of $\mu_{i}$

$$
\frac{d E_{0}\left[w_{2 i}\right]}{d \mu_{i}}-\frac{\rho}{2} V_{1}\left[w_{2 i}\right]+\lambda|\bar{U}|=0
$$

where we plug in for wealth from equation (4), use $V_{1}\left[w_{2 i}\right]=z_{i}^{2} V_{1}[\eta]$, the optimal portfolio choice (9), and the definition of near-rational expectations (5). We arrive at

$$
\frac{\mu_{i} \sigma_{\epsilon}^{2}}{\rho V_{1}[\eta]}+\frac{E_{0}\left[E_{1}[\eta] \epsilon\right]}{\rho V_{1}[\eta]}=\lambda|\bar{U}|
$$

Now we plug in the equilibrium choice $\mu_{i}=1$ and recognize that

$$
E_{0}\left[E_{1}[\eta] \epsilon\right]=\frac{\alpha_{2}}{1-\alpha_{2}} \sigma_{\epsilon}^{2}
$$

from (12) and (13). After substituting $\alpha_{2}$ by plugging (17) into (18), we get that the size of the errors relates to the costs via

$$
\sigma_{\epsilon}^{2}=\frac{V_{1}[\eta]\left(\lambda|\bar{U}| \sigma_{\nu}^{2}-V_{1}[\eta]\right)}{\sigma_{\nu}^{2}}
$$

In a last step, we sign the derivative $\frac{d \sigma_{\epsilon}^{2}}{d \lambda}$. Therefore, we re-arrange the expression we just derived to

$$
\rho \lambda|\bar{U}|=\frac{V_{1}[\eta]^{2}+\sigma_{\epsilon}^{2} \sigma_{\nu}^{2}}{V_{1}[\eta] \sigma_{\nu}^{2}}
$$


where we call $c=\lambda|\bar{U}|$. Taking the total derivative of (59) with respect to $c$ and recognizing that $V_{1}[\eta]$ is a function of $\sigma_{\epsilon}^{2}$ delivers the following expression

$$
\frac{d \sigma_{\epsilon}^{2}}{d c}=\frac{\rho V_{1}[\eta] \sigma_{\nu}^{2}}{2 V_{1}[\eta] \frac{d V_{1}[\eta]}{d \sigma_{\epsilon}^{2}}+\left(1-c \frac{d V_{1}[\eta]}{d \sigma_{\epsilon}^{2}}\right) \sigma_{\nu}^{2}} .
$$

Using (60) and the total derivative of (22) with respect to $\sigma_{\epsilon}^{2}$ delivers the lengthy expression

$$
\frac{d \sigma_{\epsilon}^{2}}{d c}=\frac{T\left(V_{1}[\eta]\right)}{c \sigma_{\nu}^{2}\left(\sigma_{\eta}^{4} \sigma_{\nu}^{2} V_{1}(\eta)^{3}+\sigma_{\eta}^{4} V_{1}(\eta)^{4}+2 \sigma_{\eta}^{2} \sigma_{\nu}^{4} V_{1}(\eta)^{2} \sigma_{\epsilon}^{2}+\sigma_{\eta}^{4} \sigma_{\nu}^{4} V_{1}(\eta) \sigma_{\epsilon}^{2}+2 \sigma_{\eta}^{4} \sigma_{\nu}^{2} V_{1}(\eta)^{2} \sigma_{\epsilon}^{2}+2 \sigma_{\eta}^{2} \sigma_{\nu}^{6} \sigma_{\epsilon}^{4}+\sigma_{\eta}^{4} \sigma_{\nu}^{4} \sigma_{\epsilon}^{4}+\sigma_{\nu}^{8} \sigma_{\epsilon}^{4}\right)}
$$

where

$$
\begin{gathered}
T\left(V_{1}[\eta]\right)=\sigma_{\eta}^{4} V_{1}(\eta)^{6}+\sigma_{\nu}^{8} V_{1}(\eta)^{2} \sigma_{\epsilon}^{4}+4 \sigma_{\eta}^{2} \sigma_{\nu}^{6} V_{1}(\eta)^{2} \sigma_{\epsilon}^{4}+2 \sigma_{\eta}^{4} \sigma_{\nu}^{6} V_{1}(\eta) \sigma_{\epsilon}^{4}+2 \sigma_{\eta}^{2} \sigma_{\nu}^{4} V_{1}(\eta)^{4} \sigma_{\epsilon}^{2}+2 \sigma_{\eta}^{4} \sigma_{\nu}^{4} V_{1}(\eta)^{3} \sigma_{\epsilon}^{2} \\
+3 \sigma_{\eta}^{4} \sigma_{\nu}^{4} V_{1}(\eta)^{2} \sigma_{\epsilon}^{4}+3 \sigma_{\eta}^{4} \sigma_{\nu}^{2} V_{1}(\eta)^{4} \sigma_{\epsilon}^{2}+2 \sigma_{\eta}^{2} \sigma_{\nu}^{8} \sigma_{\epsilon}^{6}+\sigma_{\eta}^{4} \sigma_{\nu}^{6} \sigma_{\epsilon}^{6}+\sigma_{\nu}^{10} \sigma_{\epsilon}^{6}
\end{gathered}
$$

which is clearly positive.

\section{A.4 Deriving (23) and (24)}

Jointly solving (22) and (59) for $V_{1}[\eta]$ and picking the only real solution for the conditional variance yields the two closed-form solutions

$$
\sigma_{\epsilon}=\frac{1}{\sqrt{2} \sigma_{\nu}} \sqrt{\sqrt{\sigma_{\eta}^{4}(\lambda \rho \bar{U}+1)^{2}\left(2 \lambda \rho \bar{U} \sigma_{\eta}^{2} \sigma_{\nu}^{2}(\lambda \rho \bar{U}-1)+\sigma_{\eta}^{4}(\lambda \rho \bar{U}+1)^{2}+\lambda^{2} \rho^{2} \bar{U}^{2} \sigma_{\nu}^{4}\right)}+\lambda \rho \bar{U} \sigma_{\eta}^{2} \sigma_{\nu}^{2}(1-\lambda \rho \bar{U})+\sigma_{\eta}^{4}\left(-(\lambda \rho \bar{U}+1)^{2}\right)}
$$

and

$$
V_{1}[\eta]=\frac{-\sqrt{\sigma_{\eta}^{4}(\lambda \rho \bar{U}+1)^{2}\left(2 \lambda \rho \bar{U} \sigma_{\eta}^{2} \sigma_{\nu}^{2}(\lambda \rho \bar{U}-1)+\sigma_{\eta}^{4}(\lambda \rho \bar{U}+1)^{2}+\lambda^{2} \rho^{2} \bar{U}^{2} \sigma_{\nu}^{4}\right)}+\lambda \rho \bar{U} \sigma_{\eta}^{2} \sigma_{\nu}^{2}(\lambda \rho \bar{U}+1)+\sigma_{\eta}^{4}(\lambda \rho \bar{U}+1)^{2}}{2 \sigma_{\eta}^{2}(\lambda \rho \bar{U}+1)} .
$$

The solution for the conditional variance can be re-written as

$V_{1}[\eta]=-\frac{1}{2} \sigma_{\eta}^{2} \sqrt{\frac{\lambda \rho \bar{U}\left(2 \sigma_{\eta}^{2} \sigma_{\nu}^{2}(\lambda \rho \bar{U}-1)+\lambda \rho \bar{U} \sigma_{\eta}^{4}+\lambda \rho \bar{U} \sigma_{\nu}^{4}+2 \sigma_{\eta}^{4}\right)}{\sigma_{\eta}^{4}}+1}+\frac{1}{2} \lambda \rho \bar{U}\left(\sigma_{\eta}^{2}+\sigma_{\nu}^{2}\right)+\frac{\sigma_{\eta}^{2}}{2}$.

From this form, it can be directly seen that the limit of $\lambda \rightarrow 0$ results in a conditional variance of zero. Plugging this into (20) yields (24). 


\section{A.5 Proof of Proposition 1.2}

Solve (56) for $\pi_{1}$ and differentiate with respect to $V_{1}[\eta]$ to get

$$
\frac{\partial \pi_{1}}{\partial V_{1}[\eta]}=-\frac{1+\kappa \rho \sigma_{\eta}^{2}}{\sigma_{\eta}^{2}\left(1+\kappa \rho V_{1}[\eta]\right)^{2}}<0 .
$$

In addition, differentiate both sides of (22) with respect to $\sigma_{\epsilon}$ and rearrange to get

$$
\frac{\partial V_{1}[\eta]}{\partial \sigma_{\epsilon}}=\frac{2 V_{1}[\eta]^{4}}{2 \sigma_{\epsilon}^{2} V_{1}[\eta]^{3}+\sigma_{\epsilon}^{3} \sigma_{\nu}^{4}}>0 .
$$

Then, using Lemma 1.1, the fact that $\frac{\partial \pi_{1}}{\partial \lambda}=\frac{\partial \pi_{1}}{\partial V_{1}[\eta]} \frac{\partial V_{1}[\eta]}{\partial \sigma_{\epsilon}} \frac{\partial \sigma_{\epsilon}}{\partial \lambda}$ yields (25), and applying (23) yields $(26)$.

\section{A.6 Proof of Proposition 1.3}

For the first part of the proposition, differentiate both sides of (22) with respect to $\sigma_{\nu}$ and rearrange to get

$$
\frac{\partial V_{1}[\eta]}{\partial \sigma_{\nu}}=\frac{2 \sigma_{\nu}^{2} \sigma_{\epsilon}^{2} V_{1}[\eta]^{2}+4 V_{1}[\eta]^{4}}{\sigma_{\nu}^{5} \sigma_{\epsilon}^{2}+2 \sigma_{\nu} V_{1}[\eta]^{3}}>0 .
$$

Combing this with (63) proves the first equality and the inequality for strictly positive $\sigma_{\epsilon}$. The proof of the case $\lambda \rightarrow 0$ follows directly from (23).

We start with the solution for the conditional variance in (62) and re-write it in the form

$$
V_{1}[\eta]=\frac{1}{2}\left(-\sqrt{2 \lambda \rho \bar{U} \sigma_{\eta}^{2} \sigma_{\nu}^{2}(\lambda \rho \bar{U}-1)+\sigma_{\eta}^{4}(\lambda \rho \bar{U}+1)^{2}+\lambda^{2} \rho^{2} \bar{U}^{2} \sigma_{\nu}^{4}}+\sigma_{\eta}^{2}(\lambda \rho \bar{U}+1)+\lambda \rho \bar{U} \sigma_{\nu}^{2}\right) .
$$

First, note that, when taking the limit, the term under the square root independent of $\sigma_{\nu}$ can be left out and further note that the following limit is true

$$
\lim _{x \rightarrow \infty} a_{0}+a_{1} x^{2}-x \sqrt{a_{3}+a_{1}^{2} x^{2}}=a_{0}-\frac{a_{3}}{2 a_{1}} .
$$

Apply this relationship to $\lim _{\sigma_{\nu} \rightarrow \infty} V_{1}[\eta]$ where we plug (64) in for the conditional variance and we get $\lim _{\sigma_{\nu} \rightarrow \infty} V_{1}[\eta]=\sigma_{\eta}^{2}$ if $\lambda>0$. The result from the proposition immediately follows. For the case where $\lambda=0$, note that the conditional variance is zero independent of information dispersion. 


\section{A.7 Proof of Proposition 1.4}

Differentiating (59) with respect to $\sigma_{\nu}^{2}$ and plugging in the derivative of the conditional variance which we obtain by totally differentiating (22), we get

$$
\frac{d \sigma_{\epsilon}^{2}}{d \sigma_{\nu}^{2}}=\frac{\sigma_{\eta}^{4} V_{1}(\eta)^{7}+\sigma_{\eta}^{4} \sigma_{\nu}^{6} V_{1}(\eta)^{2} \sigma_{\epsilon}^{4}+\sigma_{\nu}^{4} V_{1}(\eta)^{3} \sigma_{\epsilon}^{4}\left(\sigma_{\eta}^{2}+\sigma_{\nu}^{2}\right)^{2}+2 \sigma_{\eta}^{2} \sigma_{\nu}^{2} V_{1}(\eta)^{5} \sigma_{\epsilon}^{2}\left(\sigma_{\eta}^{2}+\sigma_{\nu}^{2}\right)+\sigma_{\eta}^{4} \sigma_{\nu}^{8} \sigma_{\epsilon}^{6}}{\sigma_{\nu}^{4} V_{1}(\eta)\left(\sigma_{\eta}^{4} \sigma_{\nu}^{2} V_{1}(\eta)^{3}+\sigma_{\eta}^{4} V_{1}(\eta)^{4}+\sigma_{\eta}^{4} \sigma_{\nu}^{4} V_{1}(\eta) \sigma_{\epsilon}^{2}+2 \sigma_{\eta}^{2} \sigma_{\nu}^{2} V_{1}(\eta)^{2} \sigma_{\epsilon}^{2}\left(\sigma_{\eta}^{2}+\sigma_{\nu}^{2}\right)+\sigma_{\nu}^{4} \sigma_{\epsilon}^{4}\left(\sigma_{\eta}^{2}+\sigma_{\nu}^{2}\right)^{2}\right)} .
$$

\section{A.8 Proof of Proposition 1.5}

Plug (11) into (1) to get

$$
K=\kappa\left(\pi_{0}+\pi_{1} \eta+\gamma \epsilon-1\right) .
$$

Taking time-zero expectations of (65) and using (19) and (20) to substitute for $\pi_{0}$ yields

$$
E_{0}[K]=\kappa(\bar{\eta}-1)\left(\frac{1+\pi_{1} \kappa \rho \sigma_{\eta}^{2}}{1+\kappa \rho \sigma_{\eta}^{2}}\right)
$$

In addition, from (65) and (28), we have

$$
\operatorname{Cov}_{0}(K, \eta)=\kappa \pi_{1} \sigma_{\eta}^{2}
$$

It follows directly that $\frac{\partial E_{0}[K]}{\partial \pi_{1}}>0$ and $\frac{\partial C o v_{0}[K, \eta]}{\partial \pi_{1}}>0$. The remainder of the proof follows from Proposition 1.2.

\section{A.9 Proof of Lemma 1.6}

Combine (2), (5), (8), (7), (9), (12), and (17) to show that

$$
z_{i}-K=\frac{\nu_{i}}{\rho \sigma_{\nu}^{2}}
$$

From (1), equilibrium profits are

$$
\Pi=\kappa \frac{(Q-1)^{2}}{2} .
$$

Taking (3), plugging in (4), and substituting $\Pi$ using (68) and (1) yields

$$
U_{i}=z_{i}(\eta-Q)+\frac{K^{2}}{2 \kappa}-\frac{\rho}{2} z_{i}^{2} V_{1}[\eta]
$$


Replacing $z_{i}=\frac{\nu_{i}}{\rho \sigma_{\nu}^{2}}+K$, applying the definition $w_{a}=K(\eta-Q)+\frac{K^{2}}{2 \kappa}$, and taking time-zero expectations on both sides yields

$$
E_{0}\left[U_{i}\right]=E_{0}\left[\frac{\nu_{i}}{\rho \sigma_{\nu}^{2}}(\eta-Q)+K(\eta-Q)+\frac{K^{2}}{2 \kappa}\right]-\frac{\rho}{2} E_{0}\left[\left(\frac{\nu_{i}}{\rho \sigma_{\nu}^{2}}+K\right)^{2}\right] V_{1}[\eta] .
$$

The second equality in (29) follows from the fact that $E_{0}\left[\nu_{i}\right]=0$ and $\nu_{i}$ is uncorrelated with $\eta$, $K$, and $Q$. As a result, the first term in the left square brackets drops out and $E_{0}\left[2 \frac{\nu_{i}}{\rho \sigma_{\nu}} K\right]=0$ in the right square brackets.

The first equality follows from noting that $E_{0}\left[U_{i}\right]$ does not depend on $\nu_{i}$. It is thus independent of $i$, and we have that

$$
S W F \equiv \int_{0}^{1} E_{0}\left[U_{i}\right] d i=E_{0}\left[U_{i}\right]
$$

Finally, use (1) to substitute $Q$ out of (4):

$$
w_{a}=K(\eta-1)-\frac{K^{2}}{2 \kappa}=\left(K \eta-E_{0}[K] \bar{\eta}\right)+\left(E_{0}[K] \bar{\eta}-K\right)-\frac{K^{2}}{2 \kappa} .
$$

Taking time-zero expectations on both sides yields (30).

Next we derive the different channels of utility. The utility specification in (3) embeds a preference for early resolution of uncertainty. To see this, note that disutility from variance stems only from conditional variance and thus the timing of the arrival of information matters for welfare. More technically, we can write $E_{0}\left[U_{i}\right]=-\frac{1}{\rho} \mathbb{E}_{0}\left[\log \left(\mathbb{E}_{1}[\exp (-\rho w)]\right)\right]$. The concave transformation through the logarithm favors volatile expectations and thus gives rise to a preference for early resolution of uncertainty.

To show the economically relevant channels of information aggregation on welfare, we make two rearrangements to the specification of utility. First, we apply the law of total variance to the variance term $V_{1}\left[w_{2 i}\right]=V_{0}\left[w_{2 i}\right]-V_{0}\left[E_{1 i}\left[w_{2 i}\right]\right]$. Second, we recognize that

$$
w_{2 i}-w_{2 a}=\frac{\nu_{i}}{\rho \sigma_{\nu}^{2}}(\eta-Q)
$$

and, since $\nu_{i}$ is independent of all other shocks, the covariance $\operatorname{Cov}_{0}\left[w_{2 i}-w_{2 a}, w_{2 a}\right]$ is zero. As a result,

$$
\begin{aligned}
V_{0}\left[w_{2 i}\right] & =V_{0}\left[w_{2 a}+w_{2 i}-w_{2 a}\right] \\
& =V_{0}\left[w_{2 a}\right]+V_{0}\left[w_{2 i}-w_{2 a}\right]
\end{aligned}
$$

The different channels by which near-rationality influences social welfare can now better been 
seen when we rearrange the second term as follows

$$
\mathrm{SWF}=E_{0}\left[U_{i}\right]=\underbrace{E_{0}\left[w_{2 a}\right]}_{\text {Level effect }}-\underbrace{\frac{1}{2} \rho V_{0}\left[w_{2 a}\right]}_{\text {Variance effect }}-\underbrace{\frac{1}{2} \rho V_{0}\left[w_{2 i}-w_{2 a}\right]}_{\text {Dispersion effect }}+\underbrace{\frac{1}{2} \rho V_{0}\left[E_{1}\left[w_{2 i}\right]\right]}_{\text {Early resolution of uncertainty }}
$$

\section{A.10 Proof of Proposition 1.7}

It follows directly from the envelope theorem that

$$
\lim _{\lambda \rightarrow 0}\left[-\frac{\partial E_{0}\left[U_{i}\right]}{\partial \mu_{i}}\right]=0
$$

For the second and third equality, note that the social welfare function (29) depends on three terms: the level of expected wealth, the idiosyncratic component in the expected volatility of portfolio returns, and the aggregate component in the expected volatility of portfolio returns. We first solve each of the three components as a function of the parameters of the model and $\pi_{1}$. Equating (14) and (56) gives

$$
\gamma=\sqrt{\frac{\left(1-\pi_{1}\right) \pi_{1}^{2} \sigma_{\nu}^{2} \sigma_{\eta} 2}{\sigma_{\epsilon}^{2}\left(\pi_{1} \sigma_{\nu}^{2}+\sigma_{\eta}^{2}\left(\pi_{1} \sigma_{\nu}^{2} \kappa \rho+\left(\pi_{1}-1\right)\right)\right)}}
$$

Squaring both sides of (65) and taking expectations gives $E_{0}\left[K^{2}\right]$. Plugging $E_{0}\left[K^{2}\right]$, (66), and (67) into (30) and substituting in (57) and (69) yields

$$
\begin{aligned}
E_{0}\left[w_{a}\right]= & -\frac{1}{2} \kappa\left\{2 \bar{\eta}\left(1-\frac{\left(\pi_{1}-1\right)^{2}\left(\bar{\eta}+\kappa \rho \sigma_{\eta}^{2}\right)}{\kappa \rho \sigma_{\eta}^{2}+1}\right)+\frac{\left(\pi_{1}-1\right)^{2}\left(\bar{\eta}+\kappa \rho \sigma_{\eta}^{2}\right)^{2}}{\left(\kappa \rho \sigma_{\eta}^{2}+1\right)^{2}}\right. \\
& \left.+\left(\pi_{1}-2\right) \pi_{1}^{2} \bar{\eta}^{2}+\frac{\left(1-\pi_{1}\right) \pi_{1}^{2} \sigma_{\nu}^{2} \sigma_{\eta}^{2}}{\pi_{1} \sigma_{\nu}^{2}+\sigma_{\eta}^{2}\left(\pi_{1}\left(\sigma_{\nu}^{2} \kappa \rho+1\right)-1\right)}+\left(\pi_{1}-2\right) \pi_{1} \sigma_{\eta}^{2}-1\right\} .
\end{aligned}
$$

We can then show that

$$
\lim _{\sigma_{\epsilon} \rightarrow 0}\left[\frac{\partial E_{0}\left[w_{a}\right]}{\partial \sigma_{\epsilon}}\right]=\lim _{\sigma_{\epsilon} \rightarrow 0}\left[\frac{\partial E_{0}\left[w_{a}\right]}{\partial \pi_{1}} \frac{\partial \pi_{1}}{\partial \sigma_{\epsilon}}\right]=\frac{\kappa \sigma_{\eta}^{2}}{2\left(1+\kappa \rho \sigma_{\eta}^{2}\right)} \lim _{\sigma_{\epsilon} \rightarrow 0}\left[\frac{\partial \pi_{1}}{\partial \sigma_{\epsilon}}\right]<0,
$$

where the last equality uses (25). Using (25) and (63) from Proposition 1.2,

$$
\lim _{\sigma_{\epsilon} \rightarrow 0}\left[-\frac{1}{2 \rho \sigma_{\nu}^{2}} \frac{\partial V_{1}[\eta]}{\partial \sigma_{\epsilon}}\right]=-\frac{1}{2 \rho \sigma_{\nu}^{2}} \lim _{\sigma_{\epsilon} \rightarrow 0}\left(\frac{\partial \pi_{1}}{\partial V_{1}[\eta]}\right)^{-1} \lim _{\sigma_{\epsilon} \rightarrow 0}\left[\frac{\partial \pi_{1}}{\partial \sigma_{\epsilon}}\right]<0
$$


Similarly, taking time-zero expectations of the third term and plugging in (57), (69), and (56) yields

$$
E_{0}\left[K^{2}\right] V_{1}[\eta]=\left(1-\pi_{1}\right) \kappa^{2} \sigma_{\eta}^{2} \frac{\sigma_{\eta}^{2}\left(\frac{\kappa \rho\left(\pi_{1}^{2}(\bar{\eta}-1)^{2}-1\right)}{\kappa \rho \sigma_{\eta}^{2}+1}+\frac{\kappa \rho\left(-\left(\pi_{1}-2\right) \pi_{1}(\bar{\eta}-1)^{2}-1\right)}{\left(\kappa \rho \sigma_{\eta}^{2}+1\right)^{2}}-\frac{\left(\pi_{1}-1\right) \pi_{1}^{2} \sigma_{\nu}^{2}}{\sigma_{\eta}^{2}\left(\pi_{1}\left(\sigma_{\nu}^{2} \kappa \rho+1\right)-1\right)+\pi_{1} \sigma_{\nu}^{2}}+\pi_{1}^{2}\right)+\frac{(\bar{\eta}-2) \bar{\eta}}{\left(\kappa \rho \sigma_{\eta}^{2}+1\right)^{2}}+1}{\pi_{1} \kappa \rho \sigma_{\eta}^{2}+1} .
$$

Again taking the derivative with respect to $\sigma_{\epsilon}$, taking the limit as $\sigma_{\epsilon}$ goes to zero and using (25) yields

$$
\lim _{\sigma_{\epsilon} \rightarrow 0}\left[-\frac{\rho}{2} \frac{\partial E_{0}\left[K^{2}\right] V_{1}[\eta]}{\partial \sigma_{\epsilon}}\right]=\frac{\kappa^{2} \rho \sigma_{\eta}^{2}\left((1-\bar{\eta})^{2}+\sigma_{\eta}^{2}\right)}{2\left(1+\kappa \rho \sigma_{\eta}^{2}\right)} \lim _{\sigma_{\epsilon} \rightarrow 0}\left[\frac{\partial \pi_{1}}{\partial \sigma_{\epsilon}}\right]<0
$$

and concludes the proof.

\section{A.11 Alternative information environments}

\section{A.11.1 Dispersed Information with an Exogenous Public Signal}

This section solves the model with public signals introduced in section 1.4. We may guess that the solution for $Q$ is some linear function of $\eta, \varpi$, and $\epsilon$ :

$$
Q=\pi_{0}+\pi_{1} \eta+\pi_{2} \varpi+\gamma \epsilon
$$

where the rational expectation of $\eta$ given $Q$ and the private and public signals is

$$
E_{i t}\left(\eta_{t+1}\right)=\alpha_{0}+\alpha_{1} s_{i}+\alpha_{2} Q+\alpha_{3} g
$$

A matching coefficients approach parallel to that in section 1.1 gives

$$
\pi_{1}=\frac{\alpha_{1}+\alpha_{3}}{1-\alpha_{2}}, \pi_{2}=\frac{\alpha_{3}}{1-\alpha_{2}}, \gamma=\frac{1}{1-\alpha_{2}} .
$$

The amplification of near-rational errors is thus influenced only in so far as the presence of

public information may induce households to put less weight on the market price of capital when forming their expectations.

The vector $\left(\eta, s_{i}, Q, g\right)$ has the following variance covariance matrix:

$$
\left(\begin{array}{cccc}
\sigma_{\eta}^{2} & \sigma_{\eta}^{2} & \pi_{1} \sigma_{\eta}^{2} & \sigma_{\eta}^{2} \\
\sigma_{\eta}^{2} & \sigma_{\eta}^{2}+\sigma_{\nu}^{2} & \pi_{1} \sigma_{\eta}^{2} & \sigma_{\eta}^{2} \\
\pi_{1} \sigma_{\eta}^{2} & \pi_{1} \sigma_{\eta}^{2} & \pi_{2}^{2} \sigma_{\varpi}^{2}+\pi_{1}^{2} \sigma_{\eta}^{2}+\gamma^{2} \sigma_{\epsilon}^{2} & \pi_{2} \sigma_{\varpi}^{2}+\pi_{1} \sigma_{\eta}^{2} \\
\sigma_{\eta}^{2} & \sigma_{\eta}^{2} & \pi_{2} \sigma_{\varpi}^{2}+\pi_{1} \sigma_{\eta}^{2} & \sigma_{\varpi}^{2}+\sigma_{\eta}^{2}
\end{array}\right) .
$$




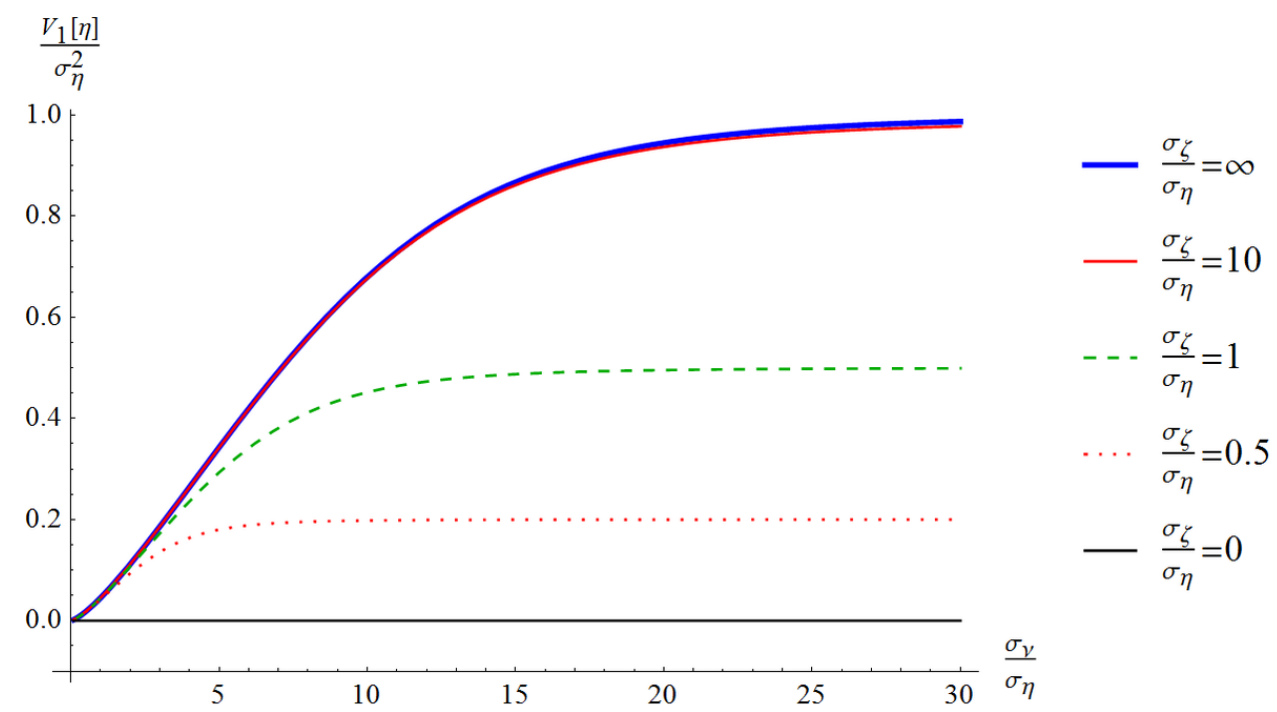

Appendix Figure 2: Ratio of the conditional variance of the productivity shock to its unconditional variance plotted over the level of dispersion of information, $\sigma_{\nu} / \sigma_{\eta}$, and for varying precisions of the public signal. In each case, $\sigma_{\epsilon} / \sigma_{\eta}$ is set to 0.01 .

Solving the signal-extraction problem returns

$$
\begin{aligned}
\alpha_{1} & =\frac{\gamma^{2} \sigma_{\varpi}^{2} \sigma_{\eta}^{2} \sigma_{\epsilon}^{2}}{\sigma_{\varpi}^{2}\left(\sigma_{\eta}^{2}\left(\gamma^{2} \sigma_{\epsilon}^{2}+\left(\pi_{1}-\pi_{2}\right)^{2} \sigma_{\nu}^{2}\right)+\gamma^{2} \sigma_{\nu}^{2} \sigma_{\epsilon}^{2}\right)+\gamma^{2} \sigma_{\eta}^{2} \sigma_{\nu}^{2} \sigma_{\epsilon}^{2}} \\
\alpha_{2} & =\frac{\left(\pi_{1}-\pi_{2}\right) \sigma_{\varpi}^{2} \sigma_{\eta}^{2} \sigma_{\nu}^{2}}{\sigma_{\varpi}^{2}\left(\sigma_{\eta}^{2}\left(\gamma^{2} \sigma_{\epsilon}^{2}+\left(\pi_{1}-\pi_{2}\right)^{2} \sigma_{\nu}^{2}\right)+\gamma^{2} \sigma_{\nu}^{2} \sigma_{\epsilon}^{2}\right)+\gamma^{2} \sigma_{\eta}^{2} \sigma_{\nu}^{2} \sigma_{\epsilon}^{2}} . \\
\alpha_{3} & =\frac{\sigma_{\eta}^{2} \sigma_{\nu}^{2}\left(\gamma^{2} \sigma_{\epsilon}^{2}+\pi_{2}\left(\pi_{2}-\pi_{1}\right) \sigma_{\varpi}^{2}\right)}{\sigma_{\varpi}^{2}\left(\sigma_{\eta}^{2}\left(\gamma^{2} \sigma_{\epsilon}^{2}+\left(\pi_{1}-\pi_{2}\right)^{2} \sigma_{\nu}^{2}\right)+\gamma^{2} \sigma_{\nu}^{2} \sigma_{\epsilon}^{2}\right)+\gamma^{2} \sigma_{\eta}^{2} \sigma_{\nu}^{2} \sigma_{\epsilon}^{2}}
\end{aligned}
$$

Based on these results, Figure 1 plots the conditional variance of $\eta$ for the rational and nearrational expectations equilibrium and for varying levels of precision of the public signal.

In the absence of near-rational behavior, the provision of public information makes no difference, because households are already fully informed from the outset. When households are near-rational, the presence of the public signal is relevant only insofar as a collapse of information aggregation affects only the subset of information that is dispersed across households and not the information that is publicly available. If the public information provided is relatively precise, $\frac{V_{1}[\eta]}{\sigma_{\eta}^{2}}$ now converges to values less than 1 as $\sigma_{\nu}$ goes to infinity.

\section{A.11.2 Dispersed Information with Aggregate Noise in Private Signal}

This subsection solves the model with aggregate noise in the private signal introduced in section 1.4. We may guess that

$$
Q=\pi_{0}+\pi_{1}(\eta+\zeta)+\gamma \epsilon
$$


where both the expectation (12) and the coefficients $\pi_{0}, \pi_{1}$, and $\gamma$ are the ones given in the main text. However, the variance-covariance matrix of the vector $\left(\eta, s_{i}, Q\right)$ changes to

$$
\left(\begin{array}{ccc}
\sigma_{\eta}^{2} & \sigma_{\eta}^{2} & \pi_{1} \sigma_{\eta}^{2} \\
\sigma_{\eta}^{2} & \sigma_{\zeta}^{2}+\sigma_{\eta}^{2}+\sigma_{\nu}^{2} & \pi_{1}\left(\sigma_{\zeta}^{2}+\sigma_{\eta}^{2}\right) \\
\pi_{1} \sigma_{\eta}^{2} & \pi_{1}\left(\sigma_{\zeta}^{2}+\sigma_{\eta}^{2}\right) & \pi_{1}^{2}\left(\sigma_{\zeta}^{2}+\sigma_{\eta}^{2}\right)+\gamma^{2} \sigma_{\epsilon}^{2}
\end{array}\right) .
$$

Applying the projection theorem yields

$$
\begin{aligned}
\alpha_{1} & =\frac{\gamma^{2} \sigma_{\eta}^{2} \sigma_{\epsilon}^{2}}{\sigma_{\zeta}^{2}\left(\gamma^{2} \sigma_{\epsilon}^{2}+\pi_{1}^{2} \sigma_{\nu}^{2}\right)+\sigma_{\eta}^{2}\left(\gamma^{2} \sigma_{\epsilon}^{2}+\pi_{1}^{2} \sigma_{\nu}^{2}\right)+\gamma^{2} \sigma_{\nu}^{2} \sigma_{\epsilon}^{2}} \\
\alpha_{2} & =\frac{\pi_{1} \sigma_{\eta}^{2} \sigma_{\nu}^{2}}{\sigma_{\zeta}^{2}\left(\gamma^{2} \sigma_{\epsilon}^{2}+\pi_{1}^{2} \sigma_{\nu}^{2}\right)+\sigma_{\eta}^{2}\left(\gamma^{2} \sigma_{\epsilon}^{2}+\pi_{1}^{2} \sigma_{\nu}^{2}\right)+\gamma^{2} \sigma_{\nu}^{2} \sigma_{\epsilon}^{2}}
\end{aligned}
$$

and

$$
V_{1}[\eta]=\frac{\sigma_{\eta}^{2}\left(\sigma_{\zeta}^{2}\left(\gamma^{2} \sigma_{\epsilon}^{2}+\pi_{1}^{2} \sigma_{\nu}^{2}\right)+\gamma^{2} \sigma_{\nu}^{2} \sigma_{\epsilon}^{2}\right)}{\sigma_{\zeta}^{2}\left(\gamma^{2} \sigma_{\epsilon}^{2}+\pi_{1}^{2} \sigma_{\nu}^{2}\right)+\sigma_{\eta}^{2}\left(\gamma^{2} \sigma_{\epsilon}^{2}+\pi_{1}^{2} \sigma_{\nu}^{2}\right)+\gamma^{2} \sigma_{\nu}^{2} \sigma_{\epsilon}^{2}}
$$

The key insight is that aggregate noise does not get amplified. Figure 3 illustrates this result. The thick blue line plots the now familiar effect of a small common error in household expectations with $\frac{\sigma_{\epsilon}}{\sigma_{\eta}}=0.01$. The red horizontal line plots the effect of an identical amount of small common noise in the private signal (i.e. $\frac{\sigma_{\zeta}}{\sigma_{\eta}}=0.01$ ). The red line has an intercept of $0.01^{2}$ and is perfectly horizontal. The common noise in the private signal is not amplified, and does the fact that an individual household observes a signal with common noise does not have an external effect on the market's capacity to aggregate information. The effect of common noise in the private signal is thus invariant to how dispersed information is in the economy.

The broken lines in Figure 3 show the same comparative static, but in the presence of large common noise in the private signal $\left(\frac{\sigma_{\zeta}}{\sigma_{\eta}}=1\right)$. Both lines retain their shape but now have a higher intercept, reflecting the fact that less information is now available to aggregate, even if the stock price is fully revealing. However, for the remaining dispersed information, the information externality of near-rational behavior operates in the same way as in the model in section 1. The externality is thus relevant whenever financial markets play an important role in aggregating dispersed information, regardless of the exact information structure. 


\section{A.11.3 Formal proof of Proposition 1.8}

Since the expressions are shorter for the case of aggregate noise in the private signal, we start with the proof for this case first. Combining (51), (52), and (72) yields

$$
\pi_{1}=\sigma_{\eta}^{2}\left(\sigma_{\zeta}^{2}+\sigma_{\eta}^{2}\right)^{-1}-2^{1 / 3} 3^{-1 / 3} \sigma_{\nu}^{2}\left(\sigma_{\zeta}^{2}+\sigma_{\eta}^{2}+\sigma_{\nu}^{2}\right) \sigma_{\epsilon}^{2} \Phi_{\zeta}^{-1}+\frac{2^{-1 / 3} 3^{-2 / 3} \Phi_{\zeta}}{\left(\sigma_{\zeta}^{2}+\sigma_{\eta}^{2}\right)^{3}}
$$

where

$$
\Phi_{\zeta}=\left(-9 \sigma_{\eta}^{2}\left(\sigma_{\zeta}^{2}+\sigma_{\eta}^{2}\right)^{5} \sigma_{\nu}^{4} \sigma_{\epsilon}^{2}+\sqrt{3} \sqrt{\left(\sigma_{\zeta}^{2}+\sigma_{\eta}^{2}\right)^{9} \sigma_{\nu}^{6} \sigma_{\epsilon}^{4}\left(27 \sigma_{\eta}^{4}\left(\sigma_{\zeta}^{2}+\sigma_{\eta}^{2}\right) \sigma_{\nu}^{2}+4\left(\sigma_{\zeta}^{2}+\sigma_{\eta}^{2}+\sigma_{\nu}^{2}\right)^{3} \sigma_{\epsilon}^{2}\right)}\right){ }^{1 / 3} .
$$

Rewriting this expression in order form of $\sigma_{\epsilon}$ yields

$$
\pi_{1}=O(1)-2^{1 / 3} 3^{-1 / 3} O(1) O\left(\sigma_{\epsilon}^{2}\right) \Phi_{\zeta}^{-1}+2^{-1 / 3} 3^{-2 / 3} O(1) \Phi_{\zeta}
$$

and

$$
\Phi_{\zeta}=\left(-9 O\left(\sigma_{\epsilon}^{2}\right)+\sqrt{3\left(O\left(\sigma_{\epsilon}^{2}\right)+4 O\left(\sigma_{\epsilon}^{6}\right)\right)}\right)^{\frac{1}{3}}=O\left(\sigma_{\epsilon}\right),
$$

where we denote $y=O(x)$ if $\frac{y}{x}=$ const as $\sigma_{\epsilon} \rightarrow 0$. Taking the derivative with respect to $\sigma_{\epsilon}$ yields

$$
\frac{\partial \pi_{1}}{\partial \sigma_{\epsilon}}=-2^{4 / 3} 3^{-1 / 3} O\left(\sigma_{\epsilon}\right) \Phi_{\zeta}^{-1}+2^{1 / 3} 3^{-1 / 3} O\left(\sigma_{\epsilon}^{2}\right) \Phi_{\zeta}^{-2} \frac{\partial \Phi_{\zeta}}{\partial \sigma_{\epsilon}}+2^{-1 / 3} 3^{-2 / 3} O(1) \frac{\partial \Phi_{\zeta}}{\partial \sigma_{\epsilon}}
$$

and

$$
\frac{\partial \Phi_{\zeta}}{\partial \sigma_{\epsilon}}=\frac{1}{3} \Phi_{\zeta}^{-2}\left(-18 O\left(\sigma_{\epsilon}\right)+\frac{1}{2}\left(O\left(\sigma_{\epsilon}^{2}\right)+4 O\left(\sigma_{\epsilon}^{6}\right)\right)^{-\frac{1}{2}}\left(2 O\left(\sigma_{\epsilon}\right)+24 O\left(\sigma_{\epsilon}^{5}\right)\right)\right) .
$$

Cancelling coefficients and taking the limit on both sides yields the proof of the first statement:

$$
\begin{aligned}
\lim _{\sigma_{\epsilon} \rightarrow 0} \frac{\partial \pi_{1}}{\partial \sigma_{\epsilon}} & =-\lim _{\sigma_{\epsilon} \rightarrow 0} O\left(\sigma_{\epsilon}\right) \Phi_{\zeta}^{-1}+\lim _{\sigma_{\epsilon} \rightarrow 0} O\left(\sigma_{\epsilon}^{2}\right) \Phi_{\zeta}^{-2} \frac{\partial \Phi_{\zeta}}{\partial \sigma_{\epsilon}}+\lim _{\sigma_{\epsilon} \rightarrow 0} \frac{\partial \Phi_{\zeta}}{\partial \sigma_{\epsilon}} \\
& =-\lim _{\sigma_{\epsilon} \rightarrow 0} O\left(\sigma_{\epsilon}\right) O\left(\sigma_{\epsilon}^{-1}\right)+\lim _{\sigma_{\epsilon} \rightarrow 0}\left(O\left(\sigma_{\epsilon}^{2}\right) O\left(\sigma_{\epsilon}^{-4}\right)+1\right) O\left(\sigma_{\epsilon}^{-2}\right)\left(-O\left(\sigma_{\epsilon}\right)+O\left(\sigma_{\epsilon}^{2}\right)\right) \\
& =-\infty
\end{aligned}
$$

The result now follows from the chain rule since $\partial \sigma_{\epsilon} / \partial \lambda>0$.

Similarly, rewriting (73) in order form of $\sigma_{\zeta}$ yields

$$
\pi_{1}=O(1) O\left(\sigma_{\zeta}^{-2}\right)-2^{1 / 3} 3^{-1 / 3} O(1) O\left(\sigma_{\zeta}^{2}\right) \Phi_{\zeta}^{-1}+2^{-1 / 3} 3^{-2 / 3} O\left(\sigma_{\zeta}^{-6}\right) \Phi_{\zeta}
$$


and

$$
\Phi_{\zeta}=\left(-9 O(1) O\left(\sigma_{\zeta}^{10}\right)+\sqrt{3\left(27 O(1) O\left(\sigma_{\zeta}^{20}\right)+4 O\left(\sigma_{\zeta}^{24}\right)\right)}\right)^{\frac{1}{3}}=O\left(\sigma_{\zeta}^{4}\right)
$$

Taking the derivative with respect to $\sigma_{\zeta}$ yields

$$
\begin{aligned}
\frac{\partial \pi_{1}}{\partial \sigma_{\zeta}}= & -O(1) O\left(\sigma_{\zeta}^{-3}\right)-2^{4 / 3} 3^{-1 / 3} O(1) O\left(\sigma_{\zeta}\right) \Phi_{\zeta}^{-1}+2^{1 / 3} 3^{-1 / 3} O(1) O\left(\sigma_{\zeta}^{2}\right) \Phi_{\zeta}^{-2} \frac{\partial \Phi_{\zeta}}{\partial \sigma_{\zeta}} \\
& -2^{2 / 3} 3^{1 / 3} O\left(\sigma_{\zeta}^{-7}\right) \Phi_{\zeta}+2^{-1 / 3} 3^{-2 / 3} O\left(\sigma_{\zeta}^{-6}\right) \frac{\partial \Phi_{\zeta}}{\partial \sigma_{\zeta}}
\end{aligned}
$$

and

$$
\frac{\partial \Phi_{\zeta}}{\partial \sigma_{\zeta}}=\frac{1}{3} \Phi_{\zeta}^{-2}\left(-90 O(1) O\left(\sigma_{\zeta}^{9}\right)+\frac{\sqrt{3}}{2}\left(27 O(1) O\left(\sigma_{\zeta}^{20}\right)+4 O\left(\sigma_{\zeta}^{24}\right)\right)^{-1 / 2}\left(540 O(1) O\left(\sigma_{\zeta}^{19}\right)+96 O\left(\sigma_{\zeta}^{23}\right)\right)\right) .
$$

The proof of the second statement follows from applying L'Hopital's rule to this expression. Because the analytical expressions become rather cumbersome, we refer the reader to the Mathematica file provided on the authors' websites for the remainder of the proof of the second statement.

For the case of the public signal, we start by combining (71) and (70) to get

$$
\begin{gathered}
\pi_{1}=27 \sigma_{\zeta}^{6} \sigma_{\eta}^{6} \sigma_{\nu}^{2} \Phi_{\varpi}^{3}+2 \sigma_{\epsilon}^{2}\left(\sigma_{\zeta}^{2} \Phi_{\varpi}\left(\sigma_{\eta}^{2}+\sigma_{\nu}^{2}\right)+\sigma_{\eta}^{2} \sigma_{\nu}^{2} \Phi_{\varpi}\right)^{3}+2 \sigma_{\epsilon}^{4}\left(\sigma_{\zeta}^{2}\left(\sigma_{\eta}^{2}+\sigma_{\nu}^{2}\right)+\sigma_{\eta}^{2} \sigma_{\nu}^{2}\right)^{6} \\
\times \frac{\sqrt[3]{2} \sqrt{3} \sigma_{\zeta} \sigma_{\eta}^{2} \sigma_{\nu}^{4} \sigma_{\epsilon}^{4 / 3}\left(2 \sqrt[3]{2} \sqrt{3} \Phi_{\varpi}^{2}+2(\sqrt{3}+3 i) \sigma_{\epsilon}^{4 / 3}\left(\sigma_{\zeta}^{2}\left(\sigma_{\eta}^{2}+\sigma_{\nu}^{2}\right)+\sigma_{\eta}^{2} \sigma_{\nu}^{2}\right)^{2}\right)}{\Phi_{\varpi}^{7}\left(27 \sigma_{\zeta}^{6} \sigma_{\eta}^{6} \sigma_{\nu}^{2}+\Phi_{\varpi}^{3}+2 \sigma_{\epsilon}^{2}\left(\sigma_{\zeta}^{2}\left(\sigma_{\eta}^{2}+\sigma_{\nu}^{2}\right)+\sigma_{\eta}^{2} \sigma_{\nu}^{2}\right)^{3}\right)}
\end{gathered}
$$

where

$$
\Phi_{\varpi}=\sqrt[3]{3 \sigma_{\zeta}^{3} \sigma_{\eta}^{3} \sigma_{\nu}\left(\sqrt{81 \sigma_{\zeta}^{6} \sigma_{\eta}^{6} \sigma_{\nu}^{2}+12 \sigma_{\epsilon}^{2}\left(\sigma_{\nu}^{2}\left(\sigma_{\zeta}^{2}+\sigma_{\eta}^{2}\right)+\sigma_{\zeta}^{2} \sigma_{\eta}^{2}\right)^{3}}-9 \sigma_{\zeta}^{3} \sigma_{\eta}^{3} \sigma_{\nu}\right)-2 \sigma_{\epsilon}^{2}\left(\sigma_{\zeta}^{2}\left(\sigma_{\eta}^{2}+\sigma_{\nu}^{2}\right)+\sigma_{\eta}^{2} \sigma_{\nu}^{2}\right)^{3}}
$$

The remaining steps mirror those of the proof for the case with aggregate noise in the private signal. In either case, the expressions are long and we refer the reader to the Mathematica file provided on the authors' websites.

\section{A.12 Comparison with Noise-Trader Model}

Consider two modifications to the model in section 1: First, households have rational expectations:

$$
\mu_{i}=0 \forall i
$$


Second, in addition to the unit interval of rational households, the economy is inhabited by a unit interval of noise traders $j \in[0,1]$ inhabit the economy. Noise traders are identical to rational households in that they have the same preferences (3), budget constraint (4), and information set (they receive the signal (2) and observe the equilibrium stock price $Q$ ). However, when making their portfolio decisions, noise traders do not maximize their utility but exogenously and inelastically demand

$$
z_{j}=\mu_{j} \vartheta
$$

where $\vartheta \sim N\left(0, \sigma_{\vartheta}^{2}\right)$. This behavior makes the supply of stocks stochastic from the perspective of rational households.

Because $\kappa=0$ implies $K=0$, market clearing requires that the sum of rational households' and noise traders' stock demands equals zero:

$$
\int_{0}^{1} z_{i} d i+\int_{0}^{1} \mu_{j} \vartheta d j=0
$$

where $\mu_{j}=1 \forall j$.

\section{Proposition A.2}

Shocks to noise-trader demand lower the utility of noise traders but raise the welfare of rational households. Noise traders' demand shocks thus represent a positive externality on rational households:

$$
\frac{\partial S W F}{\partial \sigma_{\vartheta}}>0 \forall \sigma_{\vartheta}>0 \text { and } \frac{\partial E_{0}\left[U_{j}\right]}{\partial \mu_{j}}<0 \forall \mu_{j}>0 .
$$

Proof See Appendix A.12.1.

The intuition behind this result is a redistribution of wealth between the two types of agents in the model. Although rational households incur some losses due to the increased variability of their portfolios, the market compensates them for the higher risk they take in the form of a higher risk premium. Their welfare increases because they can "lean against" noise traders' demand and thus earn higher expected returns on their investments. ${ }^{21}$ Noise-trader demand shocks thus represent a positive rather than negative externality on the welfare of rational households.

In addition, the size of this externality shrinks to 0 in the limit in which noise-trader demand shocks become small.

\section{Proposition A.3}

As the standard deviation of noise-trader demand approaches 0, its marginal effect on the elas-

\footnotetext{
${ }^{21}$ With endogenous capital accumulation $(\kappa>0)$, there also exist parameter combinations for which the deadweight loss from distortions in the capital stock outweighs the redistribution of wealth from noise traders to rational households such that the marginal effect on rational households' utility becomes negative.
} 
ticity of the stock price with respect to productivity goes to 0

$$
\lim _{\sigma_{\vartheta} \rightarrow 0} \frac{\partial \pi_{1}}{\partial \sigma_{\vartheta}}=0
$$

Proof See Appendix A.12.2.

To see the intuition for this result, replace $K$ with $\vartheta$ in (1.4). Noise-trader demand shocks are multiplied with $\frac{\rho V_{1}[\eta]}{1-\alpha_{2}}$. For small $\sigma_{\vartheta}$, both the numerator and the denominator go to 0 , such that the fraction as a whole remains a finite number. (In Appendix A.12.2, we show that the multiplier on noise traders' demand shocks is always strictly smaller than $\rho \sigma_{\nu}^{2}$.) Small common shocks to noise traders' demand thus have no first-order effect on the equilibrium informativeness of stock prices. As a result, they affect neither noise traders' own utility nor the welfare of rational households. We show in the appendix that

$$
\lim _{\sigma_{\vartheta} \rightarrow 0}\left[\frac{\partial S W F}{\partial \sigma_{\vartheta}}\right]=\lim _{\sigma_{\vartheta} \rightarrow 0}\left[\frac{\partial E_{0}\left[U_{j}\right]}{\partial \mu_{j}}\right]=0 .
$$

Small shocks to noise traders' demand thus do not give rise to the type of externality we derive in section 1. In addition, allowing for large shocks to noise-trader demand actually gives rise to a positive rather than a negative externality.

\section{A.12.1 Proof of Proposition A.2}

Because households are now fully rational, their demand schedule is

$$
z_{i}=\frac{E_{1 i}[\eta]-Q}{\rho V_{1}[\eta]}
$$

Taking time-zero expectations of (3), plugging in (4) and (76), and simplifying by law of iterated expectations yields

$$
\begin{aligned}
E_{0}\left[U_{i}\right] & =E_{0}\left[\frac{E_{1 i}[\eta-Q](\eta-Q)}{\rho V_{1}[\eta]}\right]-\frac{\rho}{2} E_{0}\left[\frac{\left(E_{1 i}[\eta-Q]\right)^{2}}{\rho^{2} V_{1}[\eta]}\right]=\frac{1}{2} E_{0}\left[\frac{\left(E_{1 i}[\eta-Q]\right)^{2}}{\rho V_{1}[\eta]}\right] \\
& =\frac{1}{2 \rho V_{1}[\eta]}\left(V_{0}\left[E_{1 i}[\eta-Q]\right]+\left(E_{0}[\eta-Q]\right)^{2}\right)
\end{aligned}
$$

where we have used that $\Pi=0$ when $\kappa=0$. Using the law of total variance, we can then replace $V_{0}\left[E_{1 i}[\eta-Q]\right]=V_{0}[\eta-Q]-V_{1}[\eta]$ and simplify to get

$$
E_{0}\left[U_{i}\right]=\frac{\left(E_{0}[\eta-Q]\right)^{2}+V_{0}[\eta-Q]}{2 \rho V_{1}[\eta]}-\frac{1}{2 \rho}=S W F,
$$


where the second equality uses the fact that $E_{0}\left[U_{i}\right]$ is no longer a function of $i$ and thus $S W F=$ $\int E_{0}\left[U_{i}\right] d i=E_{0}\left[U_{i}\right]$.

Plugging in (11) and the expressions from (77) yields

$$
S W F=\frac{1}{2} \sigma_{\nu}^{2} \sigma_{\vartheta}^{2} \rho-\frac{1}{2} \frac{\sigma_{\nu}^{6} \sigma_{\vartheta}^{4} \rho^{3}}{\sigma_{\nu}^{4} \sigma_{\vartheta}^{2} \rho^{2}+\sigma_{\eta}^{2}\left(\sigma_{\nu}^{2} \sigma_{\vartheta}^{2} \rho^{2}+1\right)} .
$$

It follows immediately that

$$
\frac{\partial S W F}{\partial \sigma_{\vartheta}}=\frac{\sigma_{\nu}^{8} \sigma_{\vartheta}^{5} \rho^{6} \sigma_{\eta}^{2}+\sigma_{\vartheta} \sigma_{\eta}^{4}\left(\sigma_{\nu}^{3} \sigma_{\vartheta}^{2} \rho^{3}+\sigma_{\nu} \rho\right)^{2}}{\rho\left(\sigma_{\eta}^{2}\left(\sigma_{\nu}^{2} \sigma_{\vartheta}^{2} \rho^{2}+1\right)+\sigma_{\nu}^{4} \sigma_{\vartheta}^{2} \rho^{2}\right)^{2}}>0 .
$$

To calculate expected utility of noise traders, again take time-zero expectations of (3), plug in (4) and (74), and simplify to get

$$
\begin{aligned}
E_{0}\left[U_{j}\right] & =E_{0}\left[\mu_{j} \vartheta(\eta-Q)\right]-\frac{\rho}{2} E_{0}\left[\mu_{j}^{2} \vartheta^{2}\right] V_{1}[\eta] \\
& =-\mu_{j} \gamma \sigma_{\vartheta}^{2}-\frac{\rho}{2} \mu_{j}^{2} \sigma_{\vartheta}^{2} V_{1}[\eta]
\end{aligned}
$$

Taking the derivative with respect to $\mu_{j}$ yields

$$
\frac{\partial E_{0}\left[U_{j}\right]}{\partial \mu_{j}}=-\gamma \sigma_{\vartheta}^{2}-\rho \mu_{j} \sigma_{\vartheta}^{2} V_{1}[\eta]<0
$$

\section{A.12.2 Proof of Proposition A.3}

Substituting $E_{1 i}[\eta]$ in (76) with $E_{1 i}[\eta]=\alpha_{0}+\alpha_{1} s_{i}+\alpha_{2} Q$ and (2), plugging the resulting expression into (75), and simplifying yields

$$
\alpha_{0}+\alpha_{1}\left(\eta+\int_{0}^{1} \nu_{i} d i\right)+\left(\alpha_{2}-1\right) Q=\rho V_{1}[\eta] \vartheta .
$$

Solving this expression for $Q$ and matching coefficients with (11) yields

$$
\pi_{0}=\frac{\alpha_{0}}{1-\alpha_{2}}, \pi_{1}=\frac{\alpha_{1}}{1-\alpha_{2}}, \gamma=\frac{\rho V_{1}[\eta]}{1-\alpha_{2}}
$$

Note that the expressions $\pi_{0}$ and $\pi_{1}$ are identical to (A.1) and (51). Similarly, repeating the steps in section 1.1, we find that the expressions for (16), 17, and (18) are identical to those in the near-rational model. However, the expression for $\gamma$ is now multiplied with $\rho V_{1}[\eta]$ relative to 
its counterpart in (52). Solving the system yields

$$
\pi_{0}=\frac{\sigma_{\eta}^{-2} \bar{\eta}}{\sigma_{\eta}^{-2}+\sigma_{\nu}^{-2}+\rho^{-2} \sigma_{\vartheta}^{-2} \sigma_{\nu}^{-4}}, \pi_{1}=\frac{\sigma_{\nu}^{-2}+\rho^{-2} \sigma_{\vartheta}^{-2} \sigma_{\nu}^{-4}}{\sigma_{\eta}^{-2}+\sigma_{\nu}^{-2}+\rho^{-2} \sigma_{\vartheta}^{-2} \sigma_{\nu}^{-4}}, \gamma=\rho \sigma_{\nu}^{2} \pi_{1} .
$$

Taking the derivative of $\pi_{1}$ with respect to $\sigma_{\vartheta}$ in (77) and simplifying yields

$$
\frac{\partial \pi_{1}}{\partial \sigma_{\vartheta}}=-\frac{2 \sigma_{\nu}^{4} \sigma_{\vartheta} \rho^{2} \sigma_{\eta}^{2}}{\left(\sigma_{\eta}^{2}\left(\sigma_{\nu}^{2} \sigma_{\vartheta}^{2} \rho^{2}+1\right)+\sigma_{\nu}^{4} \sigma_{\vartheta}^{2} \rho^{2}\right)^{2}}
$$

As $\sigma_{\vartheta}$ approaches 0 the denominator approaches $\sigma_{\eta}^{4}$ while the numerator approaches 0 .

\section{A.13 Errors about Higher Moments}

Rather than making near-rational errors about the conditional mean of $\eta$, we may consider a model identical to the one in section 1 , but in which households make a small common error about the second conditional moment rather than about the first conditional moment. We could then rewrite the market clearing condition as

$$
\frac{\alpha_{0}+\alpha_{1} \int s_{i} d i+\alpha_{2} Q-Q}{\rho V_{1}[\eta]+\epsilon_{V}}=K
$$

Solving for $Q$ yields

$$
\frac{\alpha_{0}-K \rho V_{1}[\eta]}{1-\alpha_{2}}+\frac{\alpha_{1}}{1-\alpha_{2}} \eta-\frac{K}{1-\alpha_{2}} \epsilon_{V}=Q
$$

In a model with an exogenous and strictly positive supply of capital, near-rational errors about the first and second conditional moments are thus isomorphic. However, with an endogenous capital stock, errors about the second conditional moment break the Gaussian structure of the model and are more complicated to analyze.

\section{A.14 Benefits of Observing Mistakes}

A guiding principle in our analysis of a near-rational household's incentive to become fully rational in section 1 was that households have the same information set, regardless of whether they behave fully rationally or near-rationally. In particular, a rational household can condition its decisions on $s_{i}$ and $Q$, but does not know the small correlated error it would have made, had it been near-rational.

We can relax this assumption by considering the willingness to pay of a rational household at $t=0$ for observing $\epsilon+\hat{\epsilon}_{i}$ at $t=1$. A rational household can benefit from observing this error by extracting the information it conveys about $\eta$ (and equivalently about the common component 
in the error, $\epsilon)$. Using (11), we can define

$$
\hat{s}_{i} \equiv \frac{Q-\gamma\left(\epsilon+\hat{\epsilon}_{i}\right)-\pi_{0}}{\pi_{1}}=\eta-\frac{\gamma \hat{\epsilon}_{i}}{\pi_{1}}
$$

where $\hat{s}_{i}$ is the un-biased signal about $\eta$ conveyed by $\epsilon+\hat{\epsilon}_{i}$.

\section{Proposition A.4}

As the standard deviation of the near-rational error goes to 0, a rational household's willingness to pay to observe the near-rational error it would have made had it been near-rational goes to

$$
\lim _{\sigma_{\epsilon} \rightarrow 0}\left[E_{0}\left[\left.U_{i}\right|_{\mu_{i}=0, \hat{s}_{i}}\right]-E_{0}\left[\left.U_{i}\right|_{\mu_{i}=0}\right]\right]=\frac{1}{2 \hat{\mu}^{2}}
$$

Proof See Appendix A.14.1.

The potential gain of observing this additional signal thus goes to one half of the ratio of common variance to idiosyncratic variance in the error in household expectations. Since none of the results in section 1 place restrictions on $\hat{\mu}$, the potential incentive to observe $\epsilon+\hat{\epsilon}_{i}$ is thus small for a large range of plausible parameters.

\section{A.14.1 Proof of proposition A.4}

\section{Lemma A.5}

$A$ rational household would pay

$$
E_{0}\left[\left.U_{i}\right|_{\mu_{i}=0, \hat{s}_{i}}\right]-E_{0}\left[\left.U_{i}\right|_{\mu_{i}=0}\right]=\frac{\pi_{1}^{2}\left(\left(\left(\pi_{1}-1\right) \bar{\eta}+\pi_{0}\right)^{2}+\gamma^{2} \sigma_{\epsilon}^{2}+\left(\pi_{1}-2\right) \pi_{1} \sigma_{\eta}^{2}+\sigma_{\eta}^{2}\right)}{2 \gamma^{2} \sigma_{\epsilon}^{2} \hat{\mu}^{2}}
$$

to observe the near-rational error it would have made, had it been near-rational.

Proof First, a household using additional signal $\hat{s}_{i}$ has a conditional variance of

$$
V\left[\eta \mid s_{i}, Q, \hat{s}_{i}\right] \equiv \hat{V}_{1}[\eta]=\left(\sigma_{\eta}^{-2}+\sigma_{\nu}^{-2}+\pi_{1}^{2} \gamma^{-2} \sigma_{\epsilon}^{-2}\left(1+\hat{\mu}^{-2}\right)\right)^{-1}
$$

and holds the posterior expectation

$$
E\left[\eta \mid s_{i}, Q, \hat{s}_{i}\right] \equiv \hat{E}_{i 1}[\eta]=\frac{\sigma_{\eta}^{-2} \bar{\eta}+\sigma_{\nu}^{-2} s_{i}+\pi_{1}^{2} \sigma_{\epsilon}^{-2} \gamma^{-2}\left(\eta+\frac{\gamma}{\pi_{1}} \epsilon\right)+\pi_{1}^{2} \sigma_{\epsilon}^{-2} \gamma^{-2} \hat{\mu}^{-2} \hat{s}_{i}}{\hat{V}_{1}[\eta]^{-1}}
$$

Second, plugging (4) into (3), taking time-zero expectations, and rearranging yields

$$
E_{0}\left[\left.U_{i}\right|_{\mu_{i}=0, \hat{s}_{i}}\right]=E_{0}\left[z_{i}(\eta-Q)+\Pi\right]-\frac{\rho}{2} E_{0}\left[z_{i}^{2}\right] \hat{V}_{1}[\eta],
$$


where $z_{i}=\frac{\hat{E}_{1 i}[\eta]-Q}{\rho \hat{V}_{1}[\eta]}$ from (9). It follows that a rational household's willingness to pay to observe $\hat{s}_{i}$ is

$$
\begin{aligned}
E_{0}\left[\left.U_{i}\right|_{\mu_{i}=0, \hat{s}_{i}}\right]-E_{0}\left[\left.U_{i}\right|_{\mu_{i}=0}\right]= & E_{0}\left[\frac{\hat{E}_{1 i}[\eta]-Q}{\rho \hat{V}_{1}[\eta]}(\eta-Q)+\kappa \frac{(Q-1)^{2}}{2}\right]-\frac{\rho}{2} E_{0}\left[\left(\frac{\hat{E}_{1 i}[\eta]-Q}{\rho \hat{V}_{1}[\eta]}\right)^{2}\right] \hat{V}_{1}[\eta] \\
& -\left(E_{0}\left[\frac{E_{1 i}[\eta]-Q}{\rho V_{1}[\eta]}(\eta-Q)+\kappa \frac{(Q-1)^{2}}{2}\right]-\frac{\rho}{2} E_{0}\left[\left(\frac{E_{1 i}[\eta]-Q}{\rho V_{1}[\eta]}\right)^{2}\right] V_{1}[\eta]\right) .
\end{aligned}
$$

Plugging in (2), (11), (14), (15), (78), (81), and (82) and applying the expectations operator yields the expression in the proof. Note that this calculation is somewhat involved.

Using this lemma, we now proof the Proposition. From (56), we have

$$
1-\pi_{1}=\frac{V_{1}[\eta]\left(\kappa \rho \sigma_{\eta}^{2}+1\right)}{\sigma_{\eta}^{2}\left(\kappa \rho V_{1}[\eta]+1\right)}
$$

Solving (22) for $V_{1}[\eta]$ yields three roots, one of which is real and in the interval $\left[0, \sigma_{\eta}^{2}\right]$ :

$$
V_{1}[\eta]=\frac{\sqrt[3]{2}\left(9 \sigma_{\eta}^{6} \sigma_{\nu}^{4} \sigma_{\epsilon}^{2}+\sqrt{3} \sqrt{\sigma_{\eta}^{6} \sigma_{\nu}^{6} \sigma_{\epsilon}^{4}\left(27 \sigma_{\eta}^{6} \sigma_{\nu}^{2}+4 \sigma_{\epsilon}^{2}\left(\sigma_{\eta}^{2}+\sigma_{\nu}^{2}\right)^{3}\right)}\right)^{2 / 3}-2 \sqrt[3]{3} \sigma_{\eta}^{2} \sigma_{\nu}^{2} \sigma_{\epsilon}^{2}\left(\sigma_{\eta}^{2}+\sigma_{\nu}^{2}\right)}{6^{2 / 3} \sigma_{\eta}^{2} \sqrt[3]{9 \sigma_{\eta}^{6} \sigma_{\nu}^{4} \sigma_{\epsilon}^{2}+\sqrt{3} \sqrt{\sigma_{\eta}^{6} \sigma_{\nu}^{6} \sigma_{\epsilon}^{4}\left(27 \sigma_{\eta}^{6} \sigma_{\nu}^{2}+4 \sigma_{\epsilon}^{2}\left(\sigma_{\eta}^{2}+\sigma_{\nu}^{2}\right)^{3}\right)}}} .
$$

From (85), we have

$$
V_{1}[\eta]=\frac{O\left(\sigma_{\epsilon}^{2}\right)}{O\left(\sigma_{\epsilon}\right)}-O\left(\sigma_{\epsilon}\right)=O\left(\sigma_{\epsilon}\right)
$$

Combining (84) and (86) yields $1-\pi_{1}=O\left(\sigma_{\epsilon}\right)$. Thus, using (57) and (69), we have

$$
\begin{aligned}
\pi_{0} & =O\left(1-\pi_{1}\right)=O\left(\sigma_{\epsilon}\right), \\
\gamma & =O\left(\sqrt{\frac{\pi_{1}\left(1-\pi_{1}\right)}{\sigma_{\epsilon}^{2}}}\right)=O\left(\sqrt{\frac{\pi_{1} \sigma_{\epsilon}}{\sigma_{\epsilon}^{2}}}\right)=O\left(\pi_{1}^{\frac{1}{2}} \sigma_{\epsilon}^{-\frac{1}{2}}\right) .
\end{aligned}
$$

With these two facts, taking the limit of (80) of Lemma A.5 as $\sigma_{\epsilon} \rightarrow 0$ yields

$$
\begin{aligned}
\lim _{\sigma_{\epsilon} \rightarrow 0}\left[E_{0}\left[\left.U_{i}\right|_{\mu_{i}=0, \hat{s}_{i}}\right]-E_{0}\left[\left.U_{i}\right|_{\mu_{i}=0}\right]\right] & =\lim _{\sigma_{\epsilon} \rightarrow 0} \frac{\pi_{1}^{2}\left(O\left(\sigma_{\epsilon}^{2}\right)+\left(\pi_{1}-2\right) \pi_{1} \sigma_{\eta}^{2}+\sigma_{\eta}^{2}\right)}{2 O\left(\frac{\pi_{1}}{\sigma_{\epsilon}}\right) \sigma_{\epsilon}^{2} \hat{\mu}^{2}}+\lim _{\sigma_{\epsilon} \rightarrow 0} \pi_{1}^{2} \frac{1}{2 \hat{\mu}^{2}} \\
& =\lim _{\sigma_{\epsilon} \rightarrow 0} \pi_{1}^{2} \frac{O\left(\sigma_{\epsilon}^{2}\right)}{2 O\left(\pi_{1} \sigma_{\epsilon}\right) \hat{\mu}^{2}}+\lim _{\sigma_{\epsilon} \rightarrow 0} \pi_{1}^{2} \frac{\left(\pi_{1}-2\right) \pi_{1} \sigma_{\eta}^{2}+\sigma_{\eta}^{2}}{2 O\left(\pi_{1} \sigma_{\epsilon}\right) \hat{\mu}^{2}}+\lim _{\sigma_{\epsilon} \rightarrow 0} \pi_{1}^{2} \frac{1}{2 \hat{\mu}^{2}}
\end{aligned}
$$

Then using (24) and simply plugging in $\pi_{1}=1$ gives (79). 


\section{B Appendix to Section 2}

\section{B.1 Equation of Motion for Capital}

Plugging (48) into (42) and integrating over individuals on both sides with market-clearing conditions (44), (45), and (46) gives

$$
Q_{t} K_{t+1}=Q_{t-1} R_{t} K_{t}-C_{t}+w_{t} N_{t}
$$

Plugging in (97), (43), and (47) yields

$$
K_{t+1}=\left(1-\delta_{k}\right) K_{t}+I_{t}-G_{t} K_{t}
$$

\section{B.2 Deriving the Equilibrium Conditions}

\section{Definition B.1}

Given a time path of shocks $\left\{\eta_{t}^{j}, \epsilon_{t}^{j}, \varpi_{t}^{j}\left\{\nu_{i t}^{j}, \hat{\epsilon}_{i t}^{j}: i \in[0,1]\right\}: j=L, S\right\}_{t=0}^{\infty}$, an equilibrium in this economy is a time path of quantities $\left\{\left\{C_{i t}, b_{i t}, n_{i t}, k_{i t}: i \in[0,1]\right\}, C_{t}, N_{t}, K_{t}, Y_{t}, I_{t}, G_{t}, R_{t}, a_{t}, \omega_{t}\right\}_{t=0}^{\infty}$, signals $\left\{s_{i t}^{j}, g_{t}^{j}: i \in[0,1]\right\}_{t=0}^{\infty}$, and prices $\left\{Q_{t}, r_{t}, d_{t}, w_{t}\right\}_{t=0}^{\infty}$ with the following properties:

1. $\left\{\left\{C_{i t}\right\},\left\{b_{i t}\right\},\left\{n_{i t}\right\},\left\{k_{i t}\right\}\right\}_{t=0}^{\infty}$ maximize households' lifetime utility (39) given the vector of prices, and the random sequences $\left\{\epsilon_{t}^{j}, \varpi_{t}^{j},\left\{\nu^{j} i t, \hat{\epsilon}_{i t}^{j}\right\}\right\}_{t=0}^{\infty}$;

2. The demand for capital and labor services solves the representative firm's maximization problem (37) given the vector of prices;

3. $\left\{I_{t}\right\}_{t=0}^{\infty}$ is the investment goods sector's optimal policy, maximizing (38) given the vector of prices;

4. $\left\{w_{t}\right\}_{t=0}^{\infty}$ clears the labor market, $\left\{Q_{t}\right\}_{t=0}^{\infty}$ clears the stock market, $\left\{r_{t}\right\}_{t=0}^{\infty}$ clears the bond market, and $\left\{d_{t}\right\}_{t=0}^{\infty}$ clears the market for capital services;

5. $\left\{Y_{t}\right\}_{t=0}^{\infty}$ is determined by the production function (33), and $\left\{K_{t}\right\}_{t=0}^{\infty},\left\{G_{t}\right\}_{t=0}^{\infty},\left\{a_{t}\right\}_{t=0}^{\infty}$, $\left\{R_{t}\right\}_{t=0}^{\infty}$, and $\left\{\omega_{t}\right\}_{t=0}^{\infty}$ evolve according to (B.1), (36), (34), (43), and (35), respectively;

6. $\left\{C_{t}, N_{t}\right\}_{t=0}^{\infty}$ are given by the identities

$$
X_{t}=\int_{0}^{1} X_{i t} d i, X=C, N
$$


After taking the ratio of the first-order conditions with respect to labor and consumption, we get the marginal rate of substitution between labor and consumption:

$$
\frac{1-o}{o} \frac{\left(1-n_{i t}\right)^{-1}}{C_{i t}^{-1}}=w_{t}
$$

The optimal choice of stock holdings is determined by the familiar asset-pricing equation,

$$
\mathcal{E}_{i t}\left[M_{i t+1} R_{t+1}\right]=1
$$

where the stochastic discount factor $M_{i, t+1}$ is given by

$$
M_{i t+1}=\delta\left(\frac{C_{i t+1}}{C_{i t}}\right)^{-1}\left(\frac{\tilde{C}_{i t+1}}{\tilde{C}_{i t}}\right)^{1-\frac{1}{\psi}}\left(\frac{U_{i t+1}}{\mathcal{E}_{i t}\left[U_{i t+1}^{1-\gamma}\right]^{\frac{1}{1-\gamma}}}\right)^{\frac{1}{\psi}-\gamma}
$$

and returns $R_{t+1}$ are defined in (43).

Similarly, by combining the first-order and envelope conditions for bonds, the optimal choice of bonds holdings is determined by

$$
\mathcal{E}_{i t}\left[M_{i t+1}\right]\left(1+r_{t}\right)-\frac{\pi^{\prime}\left(b_{i t}\right)}{o(1-\delta)\left(1-\frac{1}{\psi}\right) \tilde{C}_{i t}^{1-\frac{1}{\psi}} C_{i t}^{-1}}=1
$$

Given these conditions of optimality, capital and labor markets clear when conditions (44) and (46) hold, and the optimal consumption follows from the household's budget constraint (42).

\section{B.2.1 Detailed Derivation}

Agents maximize utility (39) subject to budget constraint (42). State variables in individual optimization are the holdings of capital and bonds, namely, $U_{i t}=U_{i t}\left(k_{i t}, b_{i t-1}\right)$. We denote the

derivatives of the value function with respect to $k_{i t}$ and $b_{i t-1}$ by $U_{i k t}$ and $U_{i b t}$ respectively. Thus the first-order conditions and envelope conditions are as follows:

First-order condition with respect to consumption:

$$
(1-\delta) \tilde{C}_{i t}^{-\frac{1}{\psi}} \tilde{C}_{i t} o C_{i t}^{-1}=\delta \mathcal{E}_{i t}\left[U_{i t+1}^{1-\gamma}\right]^{\frac{\gamma-\frac{1}{\psi}}{1-\gamma}} \mathcal{E}_{i t}\left[U_{i t+1}^{-\gamma} U_{i k t+1} \frac{1}{Q_{t}}\right]
$$

First-order condition with respect to bonds:

$$
\delta \mathcal{E}_{i t}\left[U_{i t+1}^{1-\gamma}\right]^{\frac{\gamma-\frac{1}{\psi}}{1-\gamma}} \mathcal{E}_{i t}\left[U_{i t+1}^{-\gamma}\left(U_{i k t+1} \frac{1}{Q_{t}}-U_{i b t+1}\right)\right]+\left(1-\frac{1}{\psi}\right)^{-1} \pi^{\prime}\left(b_{i t}\right)=0 .
$$


First-order condition with respect to labor:

$$
(1-\delta) \tilde{C}_{i t}^{-\frac{1}{\psi}} \tilde{C}_{i t}(1-o)\left(1-n_{i t}\right)^{-1}=\delta \mathcal{E}_{i t}\left[U_{i t+1}^{1-\gamma}\right]^{\frac{\gamma-\frac{1}{\psi}}{1-\gamma}} \mathcal{E}_{i t}\left[U_{i t+1}^{-\gamma} U_{i k t+1} \frac{w_{t}}{Q_{t}}\right]
$$

Envelope condition for capital:

$$
U_{i k t}=U_{i t}^{\frac{1}{\psi}} \delta \mathcal{E}_{i t}\left[U_{i t+1}^{1-\gamma}\right]^{\frac{\gamma-\frac{1}{\psi}}{1-\gamma}} \mathcal{E}_{i t}\left[U_{i t+1}^{-\gamma} U_{i k t+1} \frac{Q_{t-1}}{Q_{t}} R_{t}\right] .
$$

Envelope condition for bonds:

$$
U_{i b t}=U_{i t}^{\frac{1}{\psi}} \delta \mathcal{E}_{i t}\left[U_{i t+1}^{1-\gamma}\right]^{\frac{\gamma-\frac{1}{\psi}}{1-\gamma}} \mathcal{E}_{i t}\left[U_{i t+1}^{-\gamma} U_{i k t+1} \frac{1}{Q_{t}}\left(1+r_{t-1}\right)\right]
$$

Taking the ratio of first-order conditions with respect to labor (94) and consumption (92) gives (88), where $w_{t}$ is given by

$$
w_{t}=(1-\alpha) \frac{Y_{t}}{N_{t}}
$$

The first-order condition with respect to capital pins down the rental rate as

$$
d_{t}=\alpha \frac{Y_{t}}{K_{t}}
$$

Plugging the first-order condition with respect to consumption (92) into the right-hand side of the envelope condition for capital (95) gives

$$
U_{i k t}=U_{i t}^{\frac{1}{\psi}}(1-\delta) \tilde{C}_{i t}^{1-\frac{1}{\psi}} o C_{i t}^{-1} Q_{t-1} R_{t} .
$$

Iterating (98) to $t+1$, plugging $\frac{U_{i k t+1}}{Q_{t}}$ into the first-order condition with respect to consumption (92), and rearranging yields

$$
\tilde{C}_{i t}^{-\frac{1}{\psi}} \tilde{C}_{i t} o C_{i t}^{-1}=\delta \mathcal{E}_{i t}\left[U_{i t+1}^{1-\gamma}\right]^{\frac{\gamma-\frac{1}{\psi}}{1-\gamma}} \mathcal{E}_{i t}\left[U_{i t+1}^{-\gamma} U_{i t+1}^{\frac{1}{\psi}} \tilde{C}_{i t+1}^{1-\frac{1}{\psi}} o C_{i t+1}^{-1} R_{t+1}\right] .
$$

Using (90) in (99) yields (89).

Analogously, for bond holdings, combining first-order conditions with respect to bonds (93) and consumption (92) gives

$$
(1-\delta) \tilde{C}_{i t}^{-\frac{1}{\psi}} \tilde{C}_{i t} o C_{i t}^{-1}=\delta \mathcal{E}_{i t}\left[U_{i t+1}^{1-\gamma}\right]^{\frac{\gamma-\frac{1}{\psi}}{1-\gamma}} \mathcal{E}_{i t}\left[U_{i t+1}^{-\gamma} U_{i b t+1}\right]-\frac{\pi^{\prime}\left(b_{i t}\right)}{1-\frac{1}{\psi}}
$$

Combining the first-order condition with respect to consumption (92) and the envelope condition 
for bond holdings (96) gives

$$
U_{i b t}=U_{i t}^{\frac{1}{\psi}}(1-\delta) \tilde{C}_{i t}^{1-\frac{1}{\psi}} o C_{i t}^{-1}\left(1+r_{t-1}\right) .
$$

Substituting (101) into (100) for $U_{i b t+1}$ simplifies to (91).

\section{B.3 Proof of Lemma 2.1}

We proceed in three steps that demonstrate the consistency of the two statements in Lemma 2.1. To economize on notation, we show the equations in this section only for learning about one type of shock. The analysis readily extends to learning about short-run and long-run risk as carried out in our estimation.

First, individual state variables are functions of the set of commonly known state variables $S_{t}$ as they would be in a representative agent economy. Furthermore, households form beliefs about next period's innovation to productivity using their private signal and the market price of capital. Any individual choice by households $x_{i}$ (where $x$ can be consumption $c$, labor $n$, or capital holdings $\left.k^{\prime}\right)$ is thus a function of the state space $x_{i}\left(S_{i t}\right)$, where $S_{i t}=\left\{S_{t}, \hat{q}_{t}, \mathcal{E}_{i t}\left[\eta_{t+1}\right]\right\}$. Plugging this structure into our equilibrium condition results in a form

$$
g_{l}\left(S_{i t}\right)=\mathcal{E}_{i t}\left[g_{r}\left(S_{i t}, S_{i t+1}\right)\right]
$$

Note here that $S_{i t}$ contains all possible state variables in period $t$, and hence aggregate variables can be determined by a subset of this state vector as well.

Now we show that given the structure on the right-hand side of the equation, the left-hand side is a function of the state space $S_{i t}$. We replace the function inside the expectation on the right-hand side by its Taylor series:

$$
\begin{aligned}
g_{r}\left[S_{i t}, K_{t+1}, \omega_{t}, \eta_{t+1}, \varphi_{t+1}, \hat{q}_{t+1}, \mathcal{E}_{i t+1}\right] \\
=\sum_{\iota} \frac{c_{\iota}\left(S_{i t}\right)}{\boldsymbol{\iota} !}\left(K_{t+1}-K_{0}\right)^{\iota_{1}} \omega_{t}^{\iota_{2}} \eta_{t+1}^{\iota_{3}} \varphi_{t+1}^{\iota_{4}} \hat{q}_{t+1}^{\iota_{5}} \mathcal{E}_{i t+1}^{\iota_{6}},
\end{aligned}
$$

where $K_{0}$ is the level of capital at the deterministic steady state, $\mathcal{E}_{i t}=\mathcal{E}_{i t}\left[\eta_{t+1}\right], c_{\mathbf{j}}\left(S_{i t}\right)$ denotes the (state- $t$ dependent) coefficients of the Taylor series, and $\iota=\left(\iota_{1}, \iota_{2}, \iota_{3}, \iota_{4}, \iota_{5}, \iota_{6}\right)$ a multi-index for the expansion.

Now we take near-rational expectations conditional on $s_{i t}$ and $\hat{q}_{t}$. As Lemma B.2 shows, the conditional expectation is a sufficient statistic for the entire posterior distribution due to normality and a constant conditional variance. The terms depending on $K_{t+1}$ and $\omega_{t}$ are known at time $t$ and can thus be taken outside the expectations operator. Moreover, we get a series 
of terms depending on the conditional expectation of $\varphi_{t+1}$. Because $\varphi_{t+1}$ is unpredictable for an investor at time $t$ and all shocks are uncorrelated with each other, the first-order term is 0 , and all the higher-order terms depending on $\mathcal{E}_{i t}\left[\varphi_{t+1}\right]$ are just moments of the unconditional distributions of $\varphi$. The same is true for the terms depending on $\hat{q}_{t+1}$, and $\mathcal{E}_{i t+1}$. The only terms remaining inside the expectations operator are then those depending on $\eta_{t+1}$. We can thus write

$$
\begin{aligned}
\mathcal{E}_{i t}\left[g_{r}\left[S_{i t}, S_{i t+1}\right]\right] & =\sum_{\iota=0}^{\infty} \frac{\hat{c}_{\iota}\left(S_{i t}, K_{t+1}, \rho \omega_{t-1}+\eta_{t}\right)}{\iota !} \mathcal{E}_{i t}\left[\eta_{t+1}\right] \\
& =g_{l}\left(K_{t}, \omega_{t-1}, \eta_{t}, \varphi_{t}, \hat{q}_{t}, \mathcal{E}_{i t}\right)
\end{aligned}
$$

where the coefficients $\hat{c}_{\iota}\left(S_{i t}, K_{t+1}, \omega_{t}\right)$ collect all the terms depending on the $K_{t+1}$, $\omega_{t}$, and higher moments of the shocks $\eta_{t+1}$ and $\mathcal{E}_{i t+1}$. The third line follows from the second since all expectations of higher-order monomials of $\eta_{t+1}$ are known. This step again follows from the conditional normality with constant variance and known (deterministic) higher moments. Hence we only need to keep track of the expectation of the innovation to productivity but its higher conditional moments are constant.

Finally, in deriving the set of individual state variables, we notice that contingent-claims trading eliminates any meaningful distribution of capital across time, and thus show the consistency of the individual state space.

Second, we show that aggregate quantities depend on known state variables as well as the average expectation of next period's innovation to productivity $\hat{q}$. Therefore, consider an aggregate variable of the form

$$
\bar{X}(\bar{S})=\int x_{i}\left(S_{i}\right) d i
$$

where $\bar{X}$ can represent labor (as in (46)), consumption (87), or capital (44). Again, we plug in the Taylor series representation for individual state variables:

$$
\int x_{i}\left(S_{i}\right) d i=\int \sum_{\iota} \frac{c_{\iota}}{\iota !}\left(K_{t}-K_{0}\right)^{\iota_{1}} \omega_{t-1}^{\iota_{2}} \eta_{t}^{\iota_{3}} \varphi_{t}^{\iota_{4}} \hat{q}_{t}^{\iota_{5}} \mathcal{E}_{i t}^{\iota_{6}} d i
$$

Only the last term differs across households, and thus all other variables can be taken outside the integral. Integrating over individual expectations can be rewritten as

$$
\int \mathcal{E}_{i t}^{\iota} d i=\int\left(\mathcal{E}_{i t}-\hat{q}_{t}+\hat{q}_{t}\right)^{\iota} d i=\sum_{k=0}^{\iota}\left(\begin{array}{c}
\iota \\
k
\end{array}\right) \int\left(\mathcal{E}_{i t}-\hat{q}_{t}\right)^{k} d i \hat{q}_{t}^{\iota-k} .
$$

Again, all moments of $\mathcal{E}_{i t}-\hat{q}_{t}$, which only depends on $\nu_{i t}$, are known and thus the integral only depends on $\hat{q}$. Therefore, equation (103) holds. 
Using these insights, we solve the model using standard perturbation techniques. Perturbation methods approximate equilibrium policy functions by their Taylor series around the deterministic steady state. To arrive at the coefficients of the Taylor series, we bring all equilibrium conditions into the appropriate form shown in equation (102). Successively differentiating the equation, evaluating at the steady state, and solving the resulting system of equations for the coefficients in the Taylor series delivers the approximate solutions for the equilibrium policy functions and prices.

\section{B.4 Details on Signal Extraction}

\section{Lemma B.2}

Given Lemma 2.1 and Condition 2.2, households' equilibrium expectations of $\eta_{t+1}^{j}$ for $j=S, L$ are independent of the aggregate dynamics of the model. Due to the normality of conditioning variables $s_{i t}^{j}$ and $\hat{q}_{t}^{j}$ for $j=S, L$ respectively, the resulting conditional distributions are Gaussian and identical to the linear Gaussian setup in section 1.

Proof Given Lemma 2.1 and Condition 2.2, households infer $\hat{q}_{t}^{S}$ and $\hat{q}_{t}^{L}$ from asset prices and macroeconomic quantities. It follows immediately that

$$
E_{i t}\left[\eta_{t+1}^{j}\right]=E\left[\eta_{t+1}^{j} \mid s_{i t}^{S}, s_{i t}^{L}, S_{t}\right]=E\left[\eta_{t+1}^{j} \mid s_{i t}^{j}, \hat{q}_{t}^{j}\right] \quad \text { for } j \in\{S, L\}
$$

where $\hat{q}_{t}^{j}$ is defined by (49).

We can thus guess that the rational expectation of $\eta_{t+1}^{j}$ is the linear function

$$
E_{i t}\left[\eta_{t+1}^{j}\right]=\alpha_{0}^{j}+\alpha_{1}^{j} s_{i t}^{j}+\alpha_{2}^{j} \hat{q}_{t}^{j}
$$

where $\alpha_{0}^{j}, \alpha_{1}^{j}$, and $\alpha_{2}^{j}$ are the optimal weights on the prior, the private signal, and the average expectation, respectively. Substituting in (49), taking the integral across individuals, and solving for $\int E_{i t}\left[\eta_{t+1}^{j}\right] d i$ gives

$$
\int E_{i t}\left[\eta_{t+1}^{j}\right] d i=\frac{\alpha_{0}^{j}}{1-\alpha_{2}^{j}}+\frac{\alpha_{1}^{j}}{1-\alpha_{2}^{j}} \eta_{t+1}^{j}+\frac{\alpha_{2}^{j}}{1-\alpha_{2}^{j}} \epsilon_{t}^{j} .
$$

Adding $\epsilon_{t}^{j}$ on both sides of the equation, substituting (49) and simplifying yields

$$
\frac{1-\alpha_{2}^{j}}{\alpha_{1}^{j}} \hat{q}_{t}^{j}-\frac{\alpha_{0}^{j}}{\alpha_{1}^{j}}=\eta_{t+1}^{j}+\frac{1}{\alpha_{1}^{j}} \epsilon_{t}^{j} .
$$

Thus with the normality of the fundamental shock $\epsilon_{t}^{j}$ and the demand statistics $\hat{q}_{t}^{j}$, the forms 
for expectations and conditional variances following from Bayes' rule are identical to the linear setup.

\section{Appendix to Section 3}

\section{C.1 Moment Generation and Standard Errors}

For the macroeconomic and financial moments listed in Table 3 we use annual data from 1929 to 2008. For the first five moments, concerned with the dynamics of expectations, we use quarterly data from 1969 to 2008.

In Table 3, E[.], $\sigma($.$) , and cor(.,.) denote time-series means, standard deviations, and cor-$ relations, respectively. $d$ stands for the first difference in the time series (e.g., $\sigma(d y)$ stands for the standard deviation of output growth). $A C F[$.$] refers to the first-order autocorrelation. E_{\mathrm{i}}[$. denotes the one-period-ahead forecast from forecaster i, $\bar{E}[$.$] denotes the cross-sectional average$ of $E_{\mathrm{i}}$ [.], and $\sigma_{x s}($.$) denotes the time-series average of the cross-sectional standard deviation of$ one-period-ahead forecasts.

Fore example, $\sigma_{x s}\left(E_{i}[d y]\right)$ is the time-series average of the cross-sectional standard deviation in forecasted GDP growth one period ahead. Because forecasts in the data are for the current quarter rather than the current month we divide these series by factor three for consistency. This scaling is not an issue for the remaining variables as they are all calculated as ratios or correlations.

Standard errors of the moments and moment ratios are calculated by block-bootstrapping the truncated dataset from 1969 to 2008 times across years (following defaults of Stata's "bootstrap" command). In robustness checks we have also experimented with GMM standard errors and obtained similar results.

\section{C.2 Welfare Calculations}

\section{Lemma C.1}

The share increase in lifetime consumption that makes a household indifferent with respect to the implementation of a given policy experiment at time 0 can be written as

$$
\lambda=\frac{\log \left(\hat{U}_{0}\right)-\log \left(\bar{U}_{0}\right)}{o},
$$

where $\hat{U}_{0}=E_{0}\left[U\left(\left\{\hat{C}_{i t}, \hat{n}_{i t}\right\}_{t=1}^{\infty}\right)\right], \bar{U}_{0}=E_{0}\left[U\left(\left\{\bar{C}_{i t}, \bar{n}_{i t}\right\}_{t=1}^{\infty}\right)\right]$, and the sequences $\{\hat{C}, \hat{n}\}$ refer to the household's sequences of consumption and labor if the policy is implemented, and 
$\{\bar{C}, \bar{n}\}$ are the corresponding sequences if the policy is not implemented.

Proof First note that the utility function (39) is homogeneous of degree o in consumption:

$$
U\left(\left\{e^{\lambda} C_{i t}, n_{i t}\right\}_{t=1}^{\infty}\right)=e^{o \lambda} U\left(\left\{C_{i t}, n_{i t}\right\}_{t=1}^{\infty}\right)
$$

Using this property, it follows that the share increase in consumption, $\lambda$, that compensates the household for not adopting the policy can be written as

$$
\hat{U}_{0}=e^{o \lambda} \bar{U}_{0}
$$

The lemma follows from solving this equation for $\lambda$.

\section{C.3 Data Sources}

Consumption $\left(C_{t}\right)$. Per-capita consumption data are from the National Income and Product Accounts (NIPA) annual data reported by the Bureau of Economic Analysis (BEA). The data are constructed as the sum of consumption expenditures on nondurable goods and services (Table 1.1.5, Lines 5 and 6 ) deflated by corresponding price deflators (Table 1.1.9, Lines 5 and 6 ).

Physical Investment $\left(I_{t}\right)$. Per-capita physical investment data are also from the NIPA tables. We measure physical investment by fixed investment (Table 1.1.5, Line 8) minus informationprocessing equipment (Table 5.5.5, Line 3) deflated by its price deflator (Table 1.1.9, Line 8). Information-processing equipment is interpreted as investment in intangible capital and is therefore subtracted from fixed investment.

Output $\left(Y_{t}\right)$. It is the sum of total consumption and investment, that is, $C_{t}+I_{t}$. We exclude government expenditure and net export because they are not explicitly modeled in our economy.

Labor $\left(N_{t}\right)$. It is measured as the total number of full-time and part-time employees as reported in the NIPA Table 6.4. Data are annual.

Stock market return $\left(R_{t}\right)$ and Risk-free rate. $\left(r_{t}\right)$ The stock market returns are from the Fama-French dataset available online on K. French's webpage at http://mba.tuck.dartmouth.edu/pages/faculty/ken.french/ftp/F-F_Research_Data_Factors.zip. The nominal risk-free rate is measured by the annual three-month T-bill return. The real stock market returns and risk-free rate are computed by subtracting realized inflation (annual CPI through FRED) from the nominal risk-free rate. 
Tobin's Q $\left(Q_{t}\right)$. Data on Tobin's Q are from the Flow of Funds (FoF) and are obtained directly from the St. Louis Fed by dividing the variable MVEONWMVBSNNCB (Line 35 of Table B.102 in the FoF report) by TNWMVBSNNCB (Line 32 of table B.102 in the FoF report).

Forecast Data GDP and consumption forecast data for the period 1969-2010 are downloaded from the Survey of Professional Forecasters provided by the Philadelphia Federal Reserve at https://www.philadelphiafed.org/research-and-data/real-time-center/survey-of-professionalforecasters/historical-data/individual-forecasts We construct our forecasted GDP and consumption growth rates using the forecast for the current quarter, i.e. the quarter when the survey is conducted. The survey's questionnaires are usually sent out at the end of the first month of each quarter (after NIPA advance report), and the associated response deadlines are the second to third week of the middle month of each quarter. As a result the forecasters are essentially providing a 6 -week ahead forecast. For more detail please see the documentation listed on the above URL. The forecast file contains data from many forecasters that appear to forecast only on an occasional basis and in particular these forecasts often appear highly volatile. To screen out these, potentially less serious, forecasters we consider only data from forecasts that are in the sample for at least 80 consecutive quarters and fulfill the basic requirement of a rational forecaster that over a long horizon the variance of the forecast be strictly smaller than the variance of the forecasted variable. These criteria leave us with a total of 38 time series of forecasts.

\section{C.4 Estimating $\bar{\lambda}$ using tests of the Permanent Income Hypothesis}

Fuchs-Schuendeln and Hassan (2015) argue that although many studies reject the Permanent Income Hypothesis (PIH), it appears that households behave more rationally when the stakes are high. Specifically, when the welfare loss (as measured by equivalent variation) is economically large, studies tend to find support for the PIH. Fuchs-Schuendeln and Hassan (2015) calculate this equivalent variation by comparing two households. The first rationally smooths a preannounced income change (such as a bonus paid in December) over the course of the entire year. The second has the same baseline consumption, but consumes the extra income in the same period it is received. The equivalent variation is defined as the additional consumption amount that would have to be given to the second household to make it as well off as the first, expressed as a fraction of baseline consumption. In this appendix we replicate Fuchs-Schuendeln and Hassan's calculations using the same Epstein and Zin (1989) utility function used in the main text (39) and an intertemporal elasticity of substitution of $\psi=2$ as in Table 1. 
The first household's utility is given by:

$$
U^{\text {rational }}=\left((1-\delta) \sum_{t=0}^{11} \delta^{t}\left(y+\frac{x}{12}\right)^{1-\frac{1}{\psi}}+\delta^{12}\left(U^{\text {rational }}\right)^{1-\frac{1}{\psi}}\right)^{\frac{1}{1-\frac{1}{\psi}}}
$$

Where $y$ is the baseline consumption level and $x$ is the extra amount of consumption received in a natural experiment. The second household has the same baseline consumption, but consumes the extra income in the same period it is received (December). Thus, its utility is given by:

$U^{\text {hand-to-mouth }}=\left((1-\delta)\left(\sum_{t=0}^{10} \delta^{t}(y+z)^{1-\frac{1}{\psi}}+\delta^{11}(y+x+z)^{1-\frac{1}{\psi}}\right)+\delta^{12}\left(U^{\text {hand-to-mouth }}\right)^{1-\frac{1}{\psi}}\right)^{\frac{1}{1-\frac{1}{\psi}}}$

where $z$ is the additional amount of consumption we would have to give to the "hand-to-mouth" consumer such that $U^{\text {rational }}=U^{\text {hand-to-mouth }}$ and the equivalent variation as a percentage of permanent consumption is $z / y \times 100$.

In some studies, the increases in income are assumed to be permanent. In these cases we assume the change occurs in the middle of the year and the additional income $x$ accrues in the last six months. In some other cases the additional income is paid over two or three months. In each case we assume that these payments are made at the end of the year.

The results of these calculations are given in Table 1. For each of 17 published studies it gives the size of the change in income $(x)$, the baseline income $(y)$, as well as the horizon over which the additional income is paid. The last two columns show whether the study rejects the $\mathrm{PIH}$ as well as the equivalent variation as a percentage of $y$.

Below we list for each study how the values for $x$ and $y$ are calculated (see Fuchs-Schuendeln and Hassan (2015) for additional details).

- Parker (1999) (social security tax):

Assume there is a permanent change in the social security tax rate in the middle of the year.

$\mathrm{x}=34.06$ (Table 2, this is the average individual tax rates times the pre-tax monthly income of 2241 times six to calculate the value for half of a year)

$\mathrm{y}=1449$ (Table 2, average monthly expenditures of a household)

- Shea (1995):

$\mathrm{x}=83.88$ (Table 2, expected wage growth due to education times annual income divided by two to give increase to income in the middle of the year)

$\mathrm{y}=2330$ (Table 2, average annual household income deflated to 1982 US-dollars)

- Souleles (2002): 
Appendix Table 1: Studies of the Permanent Income Hypothesis (PIH) Sorted by Equivalent Variation (EV) as a Percentage of Permanent Consumption

\begin{tabular}{lccccc}
\hline \hline Paper & $x$ & $y$ & Paid over & Reject PIH & EV (\%) \\
\hline Parker (1999) (social security tax) & 34.06 & 1449 & 6 months & yes & 0.00 \\
Shea (1995) & 83.88 & 2330 & 6 months & yes & 0.00 \\
Souleles (2002) & 234 & $\frac{3587}{2}$ & 6 months & yes & 0.00 \\
Aaronson et al. (2012) & 474 & 2154 & 6 months & yes & 0.01 \\
Agarwal and Qian (2014) & 511 & 6644 & 1 month & no & 0.01 \\
Johnson et al. (2006) & 480 & $\frac{47021}{12}$ & 1 month & yes & 0.03 \\
Agarwal et al. (2007) & 300 & 1635 & 1 month & yes & 0.06 \\
Broda and Parker (2014) & 898 & $\frac{537000}{133}$ & 1 month & yes & 0.09 \\
Stephens (2008) & 2436 & 3325 & 6 months & yes & 0.09 \\
Scholnick (2013) & 4508.76 & 5379.58 & 6 months & yes & 0.11 \\
Parker et al. (2013) & 970.8 & $\frac{10601}{3}$ & 1 month & yes & 0.13 \\
Coulibaly and Li (2006) & 1662 & 1785 & 6 months & no & 0.14 \\
Parker (1999) (social security cap) & 990 & 1449 & 3 months & yes & 0.22 \\
Souleles (1999) & 874 & $\frac{3587}{2}$ & 1 month & yes & 0.37 \\
Browning and Collado (2001) & $\underline{817232}$ & 222674 & 2 months & no & 0.76 \\
Souleles (2000) & -1960 & 777.79 & 6 months & no & 1.40 \\
Hsieh (2003) & 2048 & 1786 & 1 month & no & 1.63 \\
\hline \hline
\end{tabular}

$\mathrm{x}=234$ (Average change of quarterly withholding using the WHOLDP measure times two)

$\mathrm{y}=3587 / 2$ (Table 1 , real gross households earnings in 1983 dollars)

- Aaronson, Agarwal and French (2012):

$\mathrm{x}=474$ (The permanent wage change increases earning by 237 dollar per quarter and we assume the consumer receives the wage increase in the middle of the year)

$\mathrm{y}=6462 / 3$ (Table 2 , average quarterly spending in 2006 dollars)

- Agarwal and Qian (2014):

$\mathrm{x}=511$ (Table 1, Panel A, average monthly benefit of treatment group in experiment)

$\mathrm{y}=6644$ (Table 1, Panel A, average monthly income of treatment group in 2016 dollars)

- Johnson and Parker (2006):

$\mathrm{x}=480$ (Table 1 , tax rebate for consumers with a positive tax rate)

$\mathrm{y}=47021 / 12$ (Table 1 , annual income divided by twelve)

- Agarwal, Liu and Soulles (2007):

$\mathrm{x}=300$ (page 1 , average monthly income for singles)

$\mathrm{y}=327 * 5$ (The average consumer in this study uses 327 dollars of credit per month, but the authors cite Chimerine 1997 to indicate that credit is about 20 percent of spending) 
- Broda and Parker (2014):

$\mathrm{x}=898$ (Table 2, average tax rebate given the rebate is greater than zero)

$\mathrm{y}=179^{*} 30 / 7^{*} 100 / 19$ (Table 2 , average weekly spending multiplied by $30 / 7$ to compute monthly spending. Y is scaled by 100/19 to adjust for the fact that data from the Nielson Consumer Panel does not capture all consumption goods)

- Stephens (2008):

$\mathrm{x}=2436$ (Table 1 , value of six months of vehicle loan payments)

$\mathrm{y}=3325$ (Table 1, average annual after tax income per consumer paying off vehicle loan)

- Scholnick (2013):

$\mathrm{x}=4508.76$ (Table 1 , value of the average final mortgage payment times six)

$\mathrm{y}=5379.58$ (Online Appendix, average income of treatment group families)

- Parker, Souleles, Johnson and McClelland (2013):

$\mathrm{x}=970.8$ (Table 6 , average tax rebate given the rebate is greater than zero)

$\mathrm{y}=10601 / 3$ (Table 6 , average quarterly consumption divided by three)

- Coulibaly and Li (2006):

$\mathrm{x}=1662$ (Table 1, average payment multuplied by six to compute half a year of payments)

$\mathrm{y}=1785$ (Table 1 , average consumption from sample)

- Parker 1999 (Social Security Cap):

Assume the household reaches the social security cap in the last three months of the year and does not pay any social security tax.

$\mathrm{x}=990$ (The temporary increase in income for the last three months of the year)

$\mathrm{y}=1449$ (Table 2, average monthly expenditures of a household)

- Souleles (1999):

$\mathrm{x}=874$ (Table 1, Mean real refund for households in CEX data in 1982-1984 dollars)

$\mathrm{y}=3587 / 2$ (Real gross annual earnings divided by twelve to compute monthly value from Souleles (2002))

- Browning and Collado (2001):

$\mathrm{x}=408616^{*} 4 / 14$ (Table A2, a bonus of $1 / 14$ of annual earnings is paid twice a year, so there are two months where $1 / 7$ of annual income is received)

$\mathrm{y}=668022 / 2$ (Table A2, total quarterly expenditures divided by 3 )

- Souleles (2000):

$\mathrm{x}=-1960$ (Household expeditures for college when it is positive)

$\mathrm{y}=777.79$ (There is an absence of expenditure and income data in the paper, so we calculate the equivalent variation using average quarterly spending from Johnson, Parker, and Souleles (2006).) 
- Hsieh (2003):

$\mathrm{x}=2048$ (Table 1, Alaska bonus in 1982-1984 dollars)

$\mathrm{y}=((713+1107)+(643+1109) / 2)$ (Table 1 , average monthly spending over two periods of time provided by author) 